\title{
Hydroxyl, water, ammonia, carbon monoxide, and neutral carbon towards the Sagittarius A complex
}

\section{VLA, Odin, and SEST observations *}

\author{
R. Karlsson ${ }^{1}$, Aa. Sandqvist ${ }^{1}$, A. Hjalmarson ${ }^{2}$, A. Winnberg ${ }^{2}$, K. Fathi ${ }^{1}$, U. Frisk $^{3}$, and M. Olberg ${ }^{2}$ \\ 1 Stockholm Observatory, Department of Astronomy, Stockholm University, AlbaNova University Center, 10691 Stockholm, \\ Sweden \\ e-mail: rolandk@astro.su.se \\ 2 Onsala Space Observatory, Chalmers University of Technology, 43992 Onsala, Sweden \\ 3 Omnisys Instruments AB, Solna strandväg 78, 17154 Solna, Sweden
}

Received 1 October 2012 / Accepted 11 March 2013

\begin{abstract}
Aims. The Sagittarius A complex in the Galactic centre comprises an ensemble of molecular clouds of different species with a variety of geometrical and kinematic properties. This work aims to study molecular abundances, morphology, and kinematics by comparing hydroxyl, water, carbon monoxide, ammonia, and atomic carbon and some of their isotopologues, in the $+50 \mathrm{~km} \mathrm{~s}^{-1}$ cloud, the circumnuclear disk (CND), the $+20 \mathrm{~km} \mathrm{~s}^{-1}$ cloud, the expanding molecular ring and the line-of-sight spiral arm features, including the Local/Sgr arm, the $-30 \mathrm{~km} \mathrm{~s}^{-1}$ arm, and the 3-kpc arm.

Methods. We observed the $+50 \mathrm{~km} \mathrm{~s}^{-1}$ cloud, the CND and the $+20 \mathrm{~km} \mathrm{~s}^{-1}$ cloud, and other selected positions at the Galactic centre with the VLA, and the Odin satellite. The VLA was used to map the 1665 and $1667 \mathrm{MHz} \mathrm{OH}$ lambda doublet main lines of the $\left({ }^{2} \Pi_{3 / 2}\right)$ state, and the Odin satellite was used to map the $\left.557 \mathrm{GHz} \mathrm{H} \mathrm{O}_{(10}-1_{01}\right)$ line as well as to observe the $548 \mathrm{GHz} \mathrm{H}_{2}^{18} \mathrm{O}\left(1_{10}-1_{01}\right)$ line, the $572 \mathrm{GHz} \mathrm{NH}_{3}\left(1_{0}-0_{0}\right)$ line, the $576 \mathrm{GHz} \mathrm{CO} J=5-4$ line and the $492 \mathrm{GHz} \mathrm{C}$ I $\left({ }^{3} \mathrm{P}_{1}-{ }^{3} \mathrm{P}_{0}\right)$ line. Furthermore, the SEST was used to map a $4.5 \times 6^{\prime}$ region of the $\mathrm{Sgr}$ A complex in the $220 \mathrm{GHz} \mathrm{C}^{18} \mathrm{O} J=2-1$ line.

Results. Strong $\mathrm{OH}$ absorption, $\mathrm{H}_{2} \mathrm{O}$ emission and absorption lines were seen at all observed positions, and the $\mathrm{H}_{2}^{18} \mathrm{O}$ line was detected in absorption towards the $+50 \mathrm{~km} \mathrm{~s}^{-1}$ cloud, the CND, the $+20 \mathrm{~km} \mathrm{~s}^{-1}$ cloud, the expanding molecular ring, and the 3-kpc arm. Strong $\mathrm{CO} J=5-4, \mathrm{C}^{18} \mathrm{O} J=2-1$, and neutral carbon $\mathrm{C}_{\mathrm{I}}$ emissions were seen towards the +50 and $+20 \mathrm{~km} \mathrm{~s}^{-1}$ clouds. $\mathrm{NH}_{3}$ was only detected in weak absorption originating in the line-of-sight spiral arm features. The abundances of $\mathrm{OH}$ and $\mathrm{H}_{2} \mathrm{O}$ in the +50 and $+20 \mathrm{~km} \mathrm{~s}^{-1}$ clouds reflect the different physical environments in the clouds, where shocks and star formation prevail in the $+50 \mathrm{~km} \mathrm{~s}^{-1}$ cloud and giving rise to a higher rate of $\mathrm{H}_{2} \mathrm{O}$ production there than in the $+20 \mathrm{~km} \mathrm{~s}^{-1}$ cloud. In the CND, cloud collisions and shocks are frequent, and the CND is also subject to intense UV-radiation emanating from the supermassive black hole and the central star cluster. The CND is rich in $\mathrm{H}_{2} \mathrm{O}$ and $\mathrm{OH}$, and these abundances are considerably higher than in the +50 and $+20 \mathrm{~km} \mathrm{~s}^{-1}$ clouds. We compare our estimated abundances of $\mathrm{OH}, \mathrm{H}_{2} \mathrm{O}$, and $\mathrm{NH}_{3}$ with similar and differing results for some other sources available in the literature. As compared to the quiescent cloud values of a few $\times 10^{-9}$, or lower, the $\mathrm{H}_{2} \mathrm{O}$ abundance is markedly enhanced in the front sides of the Sgr A molecular cloud cores, $(2-7) \times 10^{-8}$, as observed in absorption, and highest in the CND. A similar abundance enhancement is seen in $\mathrm{OH}$. The likely explanation is PDR chemistry including grain surface reactions, and perhaps also the influence of shocks. In the redward high-velocity line wings of the +50 and $+20 \mathrm{~km} \mathrm{~s}^{-1} \mathrm{clouds}$ and the CND, the $\mathrm{H}_{2} \mathrm{O}$ abundances are estimated to be $(1-6) \times 10^{-6}$ or higher, i.e., similar to the water abundances in outflows of the Orion KL and DR21 molecular clouds, which are said to be caused by the combined action of shock desorption from icy grain mantles and high-temperature, gas-phase shock chemistry.
\end{abstract}

Key words. Galaxy: center - ISM: clouds - ISM: molecules - Galaxy: abundances

\section{Introduction}

This work is aimed at studying molecular abundances, morphology, and kinematics in some of the prominent cloud features observed towards the Galactic centre (GC) by comparing observations of different species. Included in the study are the giant molecular clouds in the GC molecular belt, also

* Based on observations with the NRAO Very Large Array, the Swedish ESO Submillimetre Telescope SEST in Chile, and Odin which is a Swedish-led satellite project funded jointly by the Swedish National Space Board (SNSB), the Canadian Space Agency (CSA), the National Technology Agency of Finland (Tekes), and the Centre National d'Etude Spatiale (CNES). The Swedish Space Corporation was the industrial prime contractor and is also responsible for the satellite operation. called the molecular ridge, comprising the " $+50 \mathrm{~km} \mathrm{~s}^{-1}$ cloud" (M-0.02-0.07), the "+20 $\mathrm{km} \mathrm{s}^{-1}$ cloud" (M-0.13-0.08), and the molecular material between them, the circumnuclear disk (CND), the expanding molecular ring (EMR), and the three lineof-sight spiral arm features - the Local/Sgr arm, the $-30 \mathrm{~km} \mathrm{~s}^{-1}$ arm, and the 3-kpc arm.

The GC is dominated by the supermassive black hole (SMBH), and the co-located radio point source Sagittarius $\mathrm{A}^{*}$ $\left(\mathrm{Sgr} \mathrm{A}^{*}\right)$. An accurate estimation of the mass of the SMBH was made by Schödel et al. (2002), and later investigations by Ghez et al. (2008) have revised the mass to $\approx(4.5 \pm 0.4) \times 10^{6} M_{\odot}$. Around Sgr A*, there is the minispiral, Sgr A West, a conglomerate of ionized streams of gas. The CND surrounds Sgr A West, and is a structure of independent clumps of gas and dust showing a systematic rotation, with a rotation velocity of $110 \mathrm{~km} \mathrm{~s}^{-1}$ 
(Jackson et al. 1993). A vast number of studies have been made since the CND was first detected in dust emission by Becklin et al. (1982). Other features that we discuss are the blueshifted high-velocity $\mathrm{C}^{18} \mathrm{O}$ line emission towards the centre of Sgr A East that Genzel et al. (1990) detected at velocities of -80 to $20 \mathrm{~km} \mathrm{~s}^{-1}$, and the EMR which is recognized in positionvelocity diagrams as the high-velocity arcs at $\pm 100-200 \mathrm{~km} \mathrm{~s}^{-1}$ (Bally et al. 1988). These warm and high-density GC molecular clouds are intimately entwined, and they interact with the complex continuum emission of Sgr A, creating a variety of gas clouds, streams of gas, and shocked regions (e.g. Sandqvist 1974, 1989; Zylka et al. 1990).

The continuum emission of the Sgr A complex observed at $18 \mathrm{~cm}$ can be seen in Fig. 1. This emission is composed of synchrotron radiation as well as free-free and free-bound transitions of thermal electrons. The distribution of dust at $800 \mu \mathrm{m}$ has been observed by Lis \& Carlstrom (1994), and by Dowell et al. (1999) at $350 \mu \mathrm{m}$. General reviews of the GC Region have been published by Morris \& Serabyn (1996), Mezger et al. (1996), and most recently by Ferrière (2012).

At $18 \mathrm{~cm}$ wavelength the background continuum is strong at the GC. The ground state transition lines of $\mathrm{OH}$ are observed at this wavelength, and thus making $\mathrm{OH}$ a suitable specimen for absorption line surveys (e.g. Karlsson et al. 2003, and references therein).

The regions surrounding the GC are likely to contain a high flux of cosmic rays, strong shock waves, turbulent clouds and large photon-dominated regions. Therefore probably the endothermic chemical reactions $\mathrm{O}+\mathrm{H}_{2} \rightarrow \mathrm{OH}+\mathrm{H}$, and $\mathrm{OH}+$ $\mathrm{H}_{2} \rightarrow \mathrm{H}_{2} \mathrm{O}+\mathrm{H}$ dominate, but the chemical network of cosmic ray ionization of $\mathrm{H}_{2}$ eventually leading to $\mathrm{OH}_{3}^{+}, \mathrm{H}_{2} \mathrm{O}$ and $\mathrm{OH}$ cannot be excluded.

A comparison of the abundances of the two molecules may therefore serve as a useful tool to probe the chemistry of interstellar molecular clouds (Spaans et al. 1998; Vejby-Christensen et al. 1997; Neufeld et al. 2002). The ground state transition of $\mathrm{o}-\mathrm{H}_{2} \mathrm{O}\left(1_{10}-1_{01}\right)$, at $557 \mathrm{GHz}$, requires a very high density to be collisionally excited (e.g. Phillips \& Green 1995; Hjalmarson et al. 2003), while the $\mathrm{OH}$ lines are formed by absorption of lower-energy photons. The $\mathrm{o}-\mathrm{H}_{2} \mathrm{O}$ is not possible to detect from the surface of the Earth, due to the very strong absorption from atmospheric water (Phillips \& Green 1995). However, with the advent of orbiting observatories, such as the Submillimetre Wave Astronomical Satellite (SWAS), the Odin satellite, and the Herschel Space Observatory, it became possible to detect water at submm wavelengths using high spectral resolution. Observations of $\mathrm{H}_{2} \mathrm{O}$ in the $\mathrm{GC}$ have been published by Sandqvist et al. (2003) (Sgr A), Neufeld et al. (2000, 2003) and Wirström et al. (2010) (Sgr B2).

In this paper we present a comparison between $\mathrm{OH}$ and $\mathrm{H}_{2} \mathrm{O}$, at nine positions of the GC, see Fig. 1, and comparisons of $\mathrm{NH}_{3}$, $\mathrm{C}$, $\mathrm{CO}$, and $\mathrm{C}^{18} \mathrm{O}$ observations, at selected positions are also presented.

In the interstellar space, carbon monoxide and other molecules are well mixed with molecular hydrogen, $\mathrm{H}_{2}$, and carbon monoxide is the generally used molecule for studying interstellar molecular clouds. However, transitions of this molecule are often highly saturated in molecular clouds resulting in very high optical depths, which makes it less suitable for detailed studies of cloud properties. The much rarer ${ }^{12} \mathrm{C}^{18} \mathrm{O}$ isotopologue in the electrical dipole transition $J=2-1$, is generally optically thin in GC clouds and appears to trace the cold and dense gas coupled to the dust component of the cloud, the core, and the internal structure much better than does the optically thick
${ }^{12} \mathrm{C}^{16} \mathrm{O}$ line (Rodriguez-Fernández et al. 2001). Furthermore, the critical density of the $\mathrm{C}^{18} \mathrm{O} J=2-1$ transition is $2.0 \times 10^{4} \mathrm{~cm}^{-3}$ versus $4.2 \times 10^{3} \mathrm{~cm}^{-3}$, for the $\mathrm{C}^{18} \mathrm{OJ}=1-0$ transition, which makes the two $\mathrm{C}^{18} \mathrm{O}$ lines suitable for comparison in objects where both are observed. In this study we use the ratio of our $J=2-1$ observations and the $J=1-0$ observations by Lindqvist et al. (1995) of $\mathrm{C}^{18} \mathrm{O}$ to estimate the $\mathrm{C}^{18} \mathrm{O}$ excitation temperature and subsequently the hydrogen column density in the three main objects for this paper, the $+50 \mathrm{~km} \mathrm{~s}^{-1}$ cloud, the CND and the $+20 \mathrm{~km} \mathrm{~s}^{-1}$ cloud. The $\mathrm{C}^{18} \mathrm{O}$ transitions will measure smaller cloud sizes and line widths than does the $\mathrm{CO}$ molecule, and will give more accurate results on the distribution of column densities and kinematics of the clouds (Bally 1989).

The $572 \mathrm{GHz}$ ground state rotational transition of orthoammonia, o- $\mathrm{NH}_{3}\left(1_{0}-0_{0}\right)$, is not observable from the ground. It was first used in the Kuiper Airborne Observatory experiment by Keene et al. (1983) for studies of objects in Orion. Recently, satellite observations with Odin were performed for studies of Sgr B2 in the GC by Wirström et al. (2010). The $\mathrm{NH}_{3}\left(1_{0}-0_{0}\right)$, emission traces cool gas $(\$ 100 \mathrm{~K})$. In this work, $\mathrm{NH}_{3}$ was observed towards the Sgr A East rim and is compared with observations of $\mathrm{H}_{2} \mathrm{O}$ at the same positions. We also estimate $\mathrm{NH}_{3}$ column densities for the Local/Sgr arm, the $-30 \mathrm{~km} \mathrm{~s}^{-1}$ arm, and the 3-kpc arm of the Galaxy, making a comparison of $\mathrm{OH}$ and $\mathrm{NH}_{3}$ possible in these sources.

The atomic carbon dipole transition of $\mathrm{C}_{\mathrm{I}}\left({ }^{3} \mathrm{P}_{1}-{ }^{3} \mathrm{P}_{0}\right)$, and the $J=5-4$ transition of CO, were also observed with Odin towards the three main objects, and are compared with existing groundbased observations of the $\mathrm{CO} J=7-6$ and $J=4-3$ transitions (Martin et al. 2004). We have determined C I column densities for the main objects of this study.

\section{Observations}

Spectral line observations of the GC were performed by using two ground-based telescopes and one telescope on board a satellite. Four molecules, hydroxyl $(\mathrm{OH})$, water $\left(\mathrm{H}_{2} \mathrm{O}\right)$, ammonia $\left(\mathrm{NH}_{3}\right)$, carbon monoxide $(\mathrm{CO})$, and one atomic carbon $(\mathrm{C} \mathrm{I})$, were studied at certain energy transitions in molecular clouds within $15 \mathrm{pc}$ (in projection) from $\mathrm{Sgr} \mathrm{A}^{*}$. The rarer isotopologues of water $\left(\mathrm{H}_{2}^{18} \mathrm{O}\right)$, and carbon monoxide $\left({ }^{12} \mathrm{C}^{18} \mathrm{O}\right)$ were also observed in this region. The observations were made with the Very Large Array (VLA), the Odin satellite and the Swedish ESO Submillimetre Telescope (SEST). The observed positions include the $+50 \mathrm{~km} \mathrm{~s}^{-1}$ cloud, the molecular belt, the CND, and the $+20 \mathrm{~km} \mathrm{~s}^{-1}$ cloud. Furthermore, parts of the EMR, the lineof-sight spiral arm features: the Local/Sgr arm, the $-30 \mathrm{~km} \mathrm{~s}^{-1}$ arm, the 3-kpc arm - components III, IV and V in the paper by Sandqvist et al. (2003) - and the high negative velocity gas (Güsten \& Downes 1981) are seen in the data. In Fig. 1 the nine Odin observed positions are overlaid on our VLA map of the $18 \mathrm{~cm}$ continuum emission and numbered from 1 in the NE, to $9 \mathrm{SSW}$ of Sgr A*. Equatorial and Galactic coordinates for the observed positions are given in Table 1 .

\subsection{VLA observations of the $\mathrm{OH}$ ground state main transition lines}

The $\mathrm{OH}$ radical in the GC was observed in the $L-B$ and at $18 \mathrm{~cm}$ at the rest frequencies ${ }^{1}$ of 1665.402 and $1667.359 \mathrm{MHz}$ with the VLA radio interferometer in the hybrid configurations, BnA

\footnotetext{
http://www . narrabri . atnf. csiro.au/observing/ spectral.html
} 
R. Karlsson et al.: $\mathrm{OH}, \mathrm{H}_{2} \mathrm{O}, \mathrm{CO}, \mathrm{NH}_{3}$, and $\mathrm{C}_{\mathrm{I}}$ in the Sgr A complex

Table 1. Offset positions from Sgr A*, equatorial, and Galactic coordinates for the observed positions in the Sgr A complex.

\begin{tabular}{lccccc}
\hline \hline Pos. \# & Equatorial offsets from Sgr A* $\left(^{\prime \prime}, "\right)$ & $\alpha(\mathrm{B} 1950.0)$ & $\delta(\mathrm{B} 1950.0)$ & $l\left(^{\circ}\right)$ & $b\left(^{\circ}\right)$ \\
\hline $1\left(+50 \mathrm{~km} \mathrm{~s}^{-1}\right.$ cloud $)$ & $(+152,+78)$ & $17^{\mathrm{h}} 42^{\mathrm{m}} 41^{\mathrm{s}} .0$ & $-28^{\circ} 58^{\prime} 00^{\prime \prime}$ & -0.015 & -0.071 \\
2 & $(+100,-8)$ & $17^{\mathrm{h}} 42^{\mathrm{m}} 36^{\circ} .9$ & $-28^{\circ} 59^{\prime} 26^{\prime \prime}$ & -0.043 & -0.071 \\
3 & $(+75,+40)$ & $17^{\mathrm{h}} 42^{\mathrm{m}} 35^{\mathrm{s}} .0$ & $-28^{\circ} 58^{\prime} 38^{\prime \prime}$ & -0.035 & -0.058 \\
4 & $(+50,-94)$ & $17^{\mathrm{h}} 42^{\mathrm{m}} 33^{\mathrm{s}} .1$ & $-29^{\circ} 00^{\prime} 52^{\prime \prime}$ & -0.071 & -0.072 \\
$5\left(\mathrm{Sgr} \mathrm{A}^{*} / \mathrm{CND}\right)$ & $(0,0)(\mathrm{CND})$ & $17^{\mathrm{h}} 42^{\mathrm{m}} 29^{\circ} .3$ & $-28^{\circ} 59^{\prime} 18^{\prime \prime}$ & -0.056 & -0.046 \\
6 & $(0,-90)$ & $17^{\mathrm{h}} 42^{\mathrm{m}} 29.3$ & $-29^{\circ} 00^{\prime} 48^{\prime \prime}$ & -0.077 & -0.059 \\
$7\left(+20 \mathrm{~km} \mathrm{~s}^{-1}\right.$ cloud $)$ & $(0,-180)$ & $17^{\mathrm{h}} 42^{\mathrm{m}} 29.3$ & $-29^{\circ} 02^{\prime} 18^{\prime \prime}$ & -0.098 & -0.072 \\
8 & $(-31,-231)$ & $17^{\mathrm{h}} 42^{\mathrm{m}} 26.9$ & $-29^{\circ} 03^{\prime} 09^{\prime \prime}$ & -0.115 & -0.072 \\
9 & $(-62,-282)$ & $17^{\mathrm{h}} 42^{\mathrm{m}} 24.5$ & $-29^{\circ} 04^{\prime} 00^{\prime \prime}$ & -0.131 & -0.073 \\
\hline
\end{tabular}

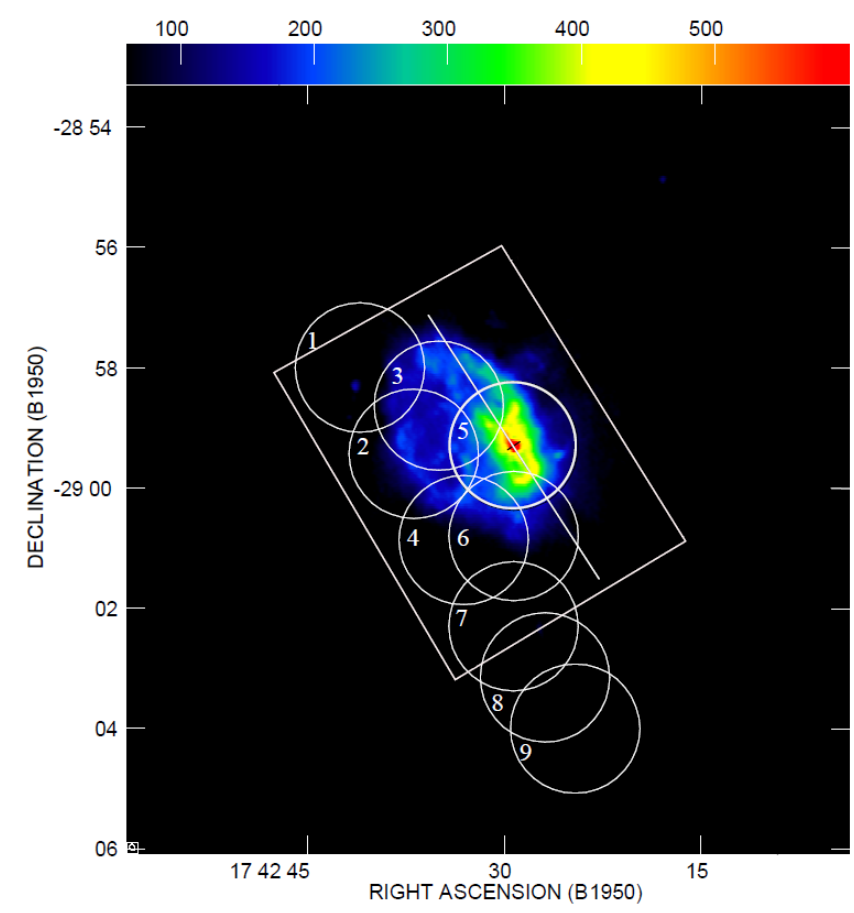

Fig. 1. VLA observations of the Sgr A complex at $18 \mathrm{~cm}$ continuum emission with a resolution of $7 " \times 5^{\prime \prime}$ (Karlsson et al., in prep.). Sgr A East is shown in blue and Sgr A West in yellow colour. The wedge scale is in $\mathrm{mJy} /$ beam. The numbered circles represent the positions and size of the Odin beam at $557 \mathrm{GHz}$. Positions 1, 5, and 7 are aiming at the $+50 \mathrm{~km} \mathrm{~s}^{-1}$ cloud, Sgr A $/ \mathrm{CND}$, and the $+20 \mathrm{~km} \mathrm{~s}^{-1}$ cloud, respectively. The white rectangle delineates the area observed by SEST, the white diagonal line is parallell to the Galactic plane, and the red spot marks the position of Sgr A*. The VLA continuum beam is marginally seen in the lower left corner of the figure, but becomes visible by "zooming".

wide array with 18 antennas, and DnC compact array with 27 antennas, in June 1986 and October 1989, respectively (Karlsson et al. 2003; and Karlsson et al., in prep.). Observational summaries are given in Table 2. The long and short baseline data were concatenated into one data set to improve sensitivity and reduce effects of missing zero-spacing observations. The compact array maps larger structures, e.g. large molecular clouds, while the wide array provides a higher angular resolution. In the concatenated data set the two aspects of observations are combined, and those data of $\mathrm{OH}$ absorption were used throughout this paper. The initial $\mathrm{OH}$ data processing was made with the NRAO AIPS programme package.

The angular resolution is $7^{\prime \prime} \times 5^{\prime \prime}$ in the concatenated data set, and the radial velocity resolution is $8.8 \mathrm{~km} \mathrm{~s}^{-1}$. The beam efficiency of the VLA in the $L$-Band was 0.55 , and was automatically corrected for by the NRAO AIPS standard calibration procedures. Line-free channels were averaged and subtracted in the $(u, v)$-plane by the NRAO AIPS programme UVLSF, and maps were CLEANed by about 3000 iterations with the NRAO AIPS MX programme using natural weighting. The typical rms noise in the concatenated data set is about $25 \mathrm{mJy} / \mathrm{beam}$. For the present study the data set was convolved with a 2.1 arcmin circular beam to resemble the Odin beam size.

\subsection{Odin submillimetre observations of $\mathrm{H}_{2} \mathrm{O}, \mathrm{H}_{2}^{18} \mathrm{O}, \mathrm{NH}_{3}$, $C O J=5-4$ and $C$ I}

Odin is a submillimetre/millimetre wave spectroscopy astronomy and aeronomy satellite launched in February of 2001. Although the design lifetime was two years, Odin is now into its thirteenth year of operation, however, now predominantly concentrating on the aeronomy part of the mission. The satellite has a high-precision 1.1-m reflector telescope with an off-axis Gregorian feed. Its main beam efficiency is $89 \%$ and the angular resolution ranges from 2.1 to 2.4 arcmin in the frequency range of 576 to $492 \mathrm{GHz}$, the range of the observations reported in this paper (Frisk et al. 2003). Its pointing uncertainty is $<15^{\prime \prime}$. Frequency calibration was performed using telluric lines as the Odin beam passed through the Earth's atmosphere during each orbit (Olberg et al. 2003). The total-power position-switching method and a duty cycle of $120 \mathrm{~s}$ was used for most of the observations, with a spectral-line-free region as the OFF-position, namely $\alpha(1950.0)=17^{\mathrm{h}} 40^{\mathrm{m}} 26^{\mathrm{s}} .8, \delta(1950.0)=-28^{\circ} 35^{\prime} 04^{\prime \prime}$. The positions of the water observations in the Sgr A complex are indicated in Fig. 1 as circles whose diameter represents the 2'.1-beam of the $557 \mathrm{GHz} \mathrm{H}_{2} \mathrm{O}$ observations. The $\mathrm{C}_{\text {I }}$ observations were performed in the Dicke sky-switching mode which implies a beam-switching against one of two sky horns with beamwidths of 4.4 , displaced $42^{\circ}$ from the main beam (Frisk et al. 2003).

Odin's receiving systems are remarkably flexible and enabled us to observe up to three different lines simultaneously. The two submillimetre channels consist of four frequencytuneable, single side band (SSB), Schottky mixer receivers with typical SSB system noise temperatures of $3300 \mathrm{~K}$. (In the position-switching mode we are limited to one channel, i.e. two submm receivers.) The backend spectrometers consist of a $1050 \mathrm{MHz}$ AOS with channel resolution of $1 \mathrm{MHz}$ and two autocorrelators (AC1, AC2) used in the $800 \mathrm{MHz}$ modes. The AOS turned out to be the most reliable spectrometer for the very wide lines typical of the GC, so it was used for all the $\mathrm{H}_{2} \mathrm{O}$ and $\mathrm{H}_{2}^{18} \mathrm{O}$ line observations. Hence, the autocorrelators were relegated to the $\mathrm{NH}_{3}, \mathrm{C}_{\mathrm{I}}$ and $\mathrm{CO} J=5-4$ observations. A summary of these observations is presented in Table 3. 
Table 2. VLA observations at $18 \mathrm{~cm}$.

\begin{tabular}{lccccc}
\hline \hline & $\begin{array}{c}\text { Frequency } \\
\text { MHz }\end{array}$ & $\begin{array}{c}\text { HPBW } \\
(\text { “ } \times ”)\end{array}$ & $\begin{array}{c}\text { PA } \\
(\mathrm{deg})\end{array}$ & $\begin{array}{c}\text { Velocity res. } \\
\left(\mathrm{km} \mathrm{s}^{-1}\right)\end{array}$ & $\begin{array}{c}t_{\text {integration }} \\
(\mathrm{min})\end{array}$ \\
\hline BnA, $R$-pol. & 1665 & $3.9 \times 2.9$ & 64.7 & 8.8 & 169 \\
BnA, $R$-pol. & 1667 & $4.0 \times 2.8$ & 61.1 & 8.8 & 173 \\
DnC, $R$ - and $L$-pol. & 1665 & $24.0 \times 22.3$ & 29.8 & 8.8 & 144 \\
DnC, $R$ - and $L$-pol. & 1667 & $30.2 \times 23.7$ & 46.3 & 8.8 & 136 \\
\hline
\end{tabular}

Notes. The telescope was centred at $17^{\mathrm{h}} 42^{\mathrm{m}} 30^{\mathrm{s}} 0,-28^{\circ} 59^{\prime} 300^{\prime} 0$ (B1950.0).

Table 3. Summary of the Odin submillimetre observations of the Sgr A complex.

\begin{tabular}{|c|c|c|c|c|c|c|}
\hline Species & Transition & $\begin{array}{c}\text { Frequency } \\
(\mathrm{GHz})\end{array}$ & $\begin{array}{c}\text { Pos. } \\
\#\end{array}$ & $\begin{array}{l}\text { Integration } \\
\text { time }(\mathrm{h})\end{array}$ & Observing dates & $\begin{array}{r}T_{\text {cont }}^{a} \\
(\mathrm{~K})\end{array}$ \\
\hline \multirow[t]{9}{*}{$\mathrm{o}-\mathrm{H}_{2} \mathrm{O}$} & $1_{1,0}-1_{0,1}$ & 556.9360 & 1 & 10.4 & April 2002 & 0.21 \\
\hline & & & 2 & 9.6 & February/March 2005 & 0.18 \\
\hline & & & 3 & 9.0 & February/March 2005 & 0.13 \\
\hline & & & 4 & 9.7 & February/March 2005 & 0.16 \\
\hline & & & 5 & 11.2 & September 2002 & 0.13 \\
\hline & & & 6 & 9.0 & February/March 2005 & 0.15 \\
\hline & & & 7 & 11.3 & April 2002 & 0.21 \\
\hline & & & 8 & 18.0 & April 2008 & 0.28 \\
\hline & & & 9 & 17.2 & March 2007 & 0.31 \\
\hline \multirow[t]{3}{*}{$\mathrm{o}-\mathrm{H}_{2}^{18} \mathrm{O}$} & $1_{1,0}-1_{0,1}$ & 547.6764 & 1 & 55.4 & February/March 2007 & 0.21 \\
\hline & & & 5 & $20.9 / 5.7 / 14.9 / 15.9$ & March 2002/Feb. 2010/April 2011/Feb. 2012 & 0.13 \\
\hline & & & 7 & $19.0 / 15.7 / 15.7$ & Sept. 2002/April 2010/April 2011 & 0.20 \\
\hline $\mathrm{o}-\mathrm{NH}_{3}$ & $1_{0}-0_{0}$ & 572.4981 & $2,3,4,6$ & 39.2 & February/March 2005 & 0.16 \\
\hline \multirow[t]{3}{*}{$\mathrm{CO}$} & $5-4$ & 576.2679 & 1 & 10.4 & April 2002 & \\
\hline & & & 5 & 11.2 & September 2002 & \\
\hline & & & 7 & 11.3 & April 2002 & \\
\hline \multirow[t]{3}{*}{$\mathrm{C}_{\mathrm{I}}$} & ${ }^{3} \mathrm{P}_{1}-{ }^{3} \mathrm{P}_{0}$ & 492.1607 & 1 & 0.7 & April 2003 & \\
\hline & & & 5 & 0.7 & April 2003 & \\
\hline & & & 7 & 0.8 & April 2003 & \\
\hline
\end{tabular}

Notes. ${ }^{(a)}$ Odin estimate (see Sect. 3.2).

\subsection{SEST observations of $\mathrm{C}^{18} \mathrm{O} \mathrm{J}=2-1$}

The observations of the $219.6 \mathrm{GHz} \mathrm{C}^{18} \mathrm{O} J=2-1$ line towards the Sgr A complex were carried out in August 1997 and August 1999 with the 15-m SEST on the La Silla mountain in Chile. A $4.5 \times 6^{\prime}$ region was mapped on a grid comparable with that defined in the $\mathrm{C}^{18} \mathrm{O} J=1-0$ survey by Lindqvist et al. (1995), but with a spacing of $22^{\prime \prime} .5$. We have used the same coordinate reference as Lindqvist et al. (1995), i.e. $(l, b)=\left(+5^{\prime}\right.$, $\left.-3^{\prime}\right)$, to facilitate comparison between the $\mathrm{C}^{18} \mathrm{O} J=1-0$ and $J=2-1$ data. The half-power beamwidth of the telescope was $24^{\prime \prime}$ and the main beam efficiency $\left(\eta_{\mathrm{mb}}\right)$ was 0.60 . The dual channel $115 / 230 \mathrm{GHz}$ superconductor-insulator-superconductor (SIS) receiver was used and the system temperature at the observed $219.6 \mathrm{GHz}$ was about $250 \mathrm{~K}$ most of the time (but could range between 200 and $400 \mathrm{~K}$, depending upon the weather and elevation). The backend was the 1440 channel $1 \mathrm{GHz}$ Low Resolution (LRS1) acousto-optical spectrometer (AOS), which yielded a velocity resolution of $1.0 \mathrm{~km} \mathrm{~s}^{-1}$ per channel. The position-switching method was used with the line-free reference OFF-position being at Galactic coordinates $(l, b)=\left(5^{\prime}, 30^{\prime}\right)$. The ON-source integration time was $10 \mathrm{~min}$ per point, at more than 180 positions, and the average rms noise became $15 \mathrm{mK}$.

\section{Results}

\subsection{Hydroxyl $(\mathrm{OH})$}

$\mathrm{OH}$ absorption is detected at all the nine positions in Fig. 1. The $\mathrm{OH}$ profiles reveal strong absorption against the $+50 \mathrm{~km} \mathrm{~s}^{-1}$ cloud, the CND and the $+20 \mathrm{~km} \mathrm{~s}^{-1}$ cloud. $\mathrm{OH}$ absorption is also detected at velocities around -170 and $-130 \mathrm{~km} \mathrm{~s}^{-1}$, corresponding to the velocities of the HNVG (Yusef-Zadeh et al. 1993) and the EMR, respectively. At velocities close to $0 \mathrm{~km} \mathrm{~s}^{-1}$ the Local/Sgr arm is identified, and furthermore, the $-30 \mathrm{~km} \mathrm{~s}^{-1}$ arm and the $3-\mathrm{kpc}$ arm are seen at velocities around -30 and $-50 \mathrm{~km} \mathrm{~s}^{-1}$, respectively. Figure 2 shows the line profiles of the 1665 and $1667 \mathrm{MHz} \mathrm{OH}$ absorption, with the $557 \mathrm{GHz} \mathrm{H}_{2} \mathrm{O}$ profiles at the nine positions superimposed. It should be noted that the $\mathrm{H}_{2} \mathrm{O}$ emission is strongly affected by self-absorption by foregound gas of low excitation where the $\mathrm{OH}$ absorption is strongest, at Pos. 1, 2, 3, and 5.

The $1667 \mathrm{MHz}$ line shows deeper absorption than does the $1665 \mathrm{MHz}$ line at all positions, which is consistent with the expected ratio for the respective lines in local thermodynamic equilibrium (LTE) conditions, and far from the optically thin ratio of $9 / 5$, i.e. the spectra reveal high optical depths. The strongest $\mathrm{OH}$ absorption at $1667 \mathrm{MHz}$ is seen at the nearmost surface of contact between the $+50 \mathrm{~km} \mathrm{~s}^{-1}$ cloud and the expanding Sgr A East shell (Pos. 3) (e.g. Herrnstein \& Ho 2005). The observed intensities of the 1667 and $1665 \mathrm{MHz}$ lines are $-50 \mathrm{Jy} /$ beam and $-42 \mathrm{Jy} /$ beam, respectively, at Pos. 3 , at the same radial velocity of $44 \mathrm{~km} \mathrm{~s}^{-1}$ for both lines. The velocity at full width at half maximum $\left(\Delta V_{\mathrm{FWHM}}\right)$ of the $+50 \mathrm{~km} \mathrm{~s}^{-1}$ cloud is estimated to be $33 \mathrm{~km} \mathrm{~s}^{-1}$. In Fig. 2 the well-known regions at different velocities are clearly seen, which are identified by multi-Gaussian fitting to the $1667 \mathrm{MHz}$ profile and shown in Fig. 3. The absorption-peak velocity decreases outside of the $+50 \mathrm{~km} \mathrm{~s}^{-1}$ cloud in the SW direction along the molecular belt. 

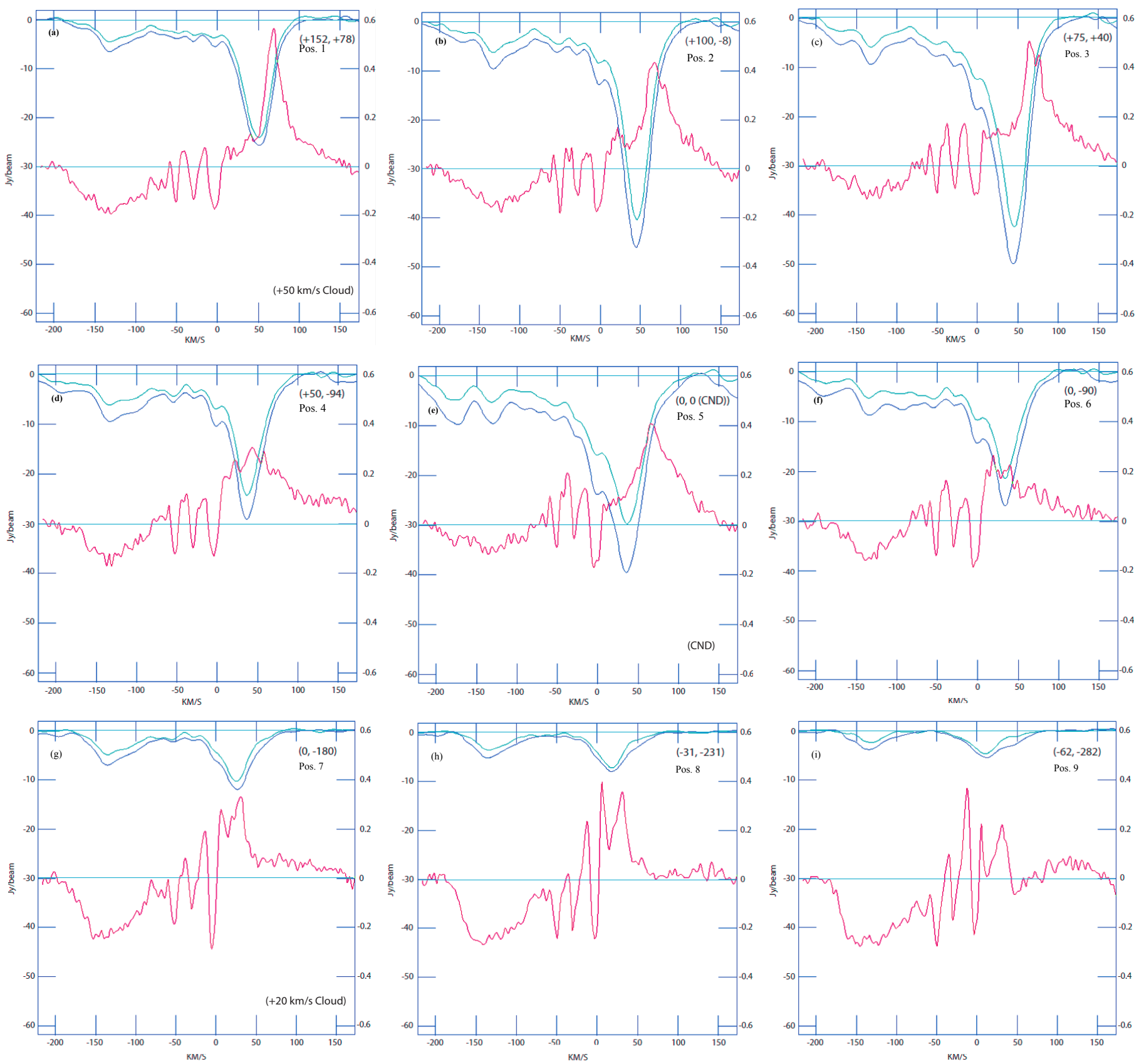

Fig. 2. 1665 and $1667 \mathrm{MHz} \mathrm{OH}$ absorption and the $\mathrm{H}_{2} \mathrm{O}$ profiles towards the Sgr A complex. The upper (cyan) line profiles refer to the $1665 \mathrm{MHz}$ $\mathrm{OH}$ line, and the deeper (blue) absorption profiles belong to the $1667 \mathrm{MHz} \mathrm{OH}$ line. The (red) profiles in the middle of the diagrams are the $\mathrm{H}_{2} \mathrm{O}$ profiles. The scale on the left side of the diagrams ( 0 to $\left.-60 \mathrm{Jy} / \mathrm{beam}\right)$ corresponds to the $\mathrm{OH}$ absorption, and the scale on the right side $( \pm 0.6 \mathrm{~K})$ is the antenna temperature of the $\mathrm{H}_{2} \mathrm{O}$ profiles. The equatorial offset coordinates from Sgr A* are given in the upper right corner in each figure. Our high resolution $\mathrm{OH}$ data have been convolved here with a $126^{\prime \prime}$ circular beam to resemble the angular resolution of the $O$ din $\mathrm{H}_{2} \mathrm{O}$ data.

There is also strong absorption at Pos. 5, towards the CND, of $-40 \mathrm{Jy} / \mathrm{beam}$ at $1667 \mathrm{MHz}$, and the line is wide with a $\Delta V_{\mathrm{FWHM}}$ of $84 \mathrm{~km} \mathrm{~s}^{-1}$. Signatures of the Local/Sgr arm around $0 \mathrm{~km} \mathrm{~s}^{-1}$, the $-30 \mathrm{~km} \mathrm{~s}^{-1}$ arm, and the 3-kpc arm at about $-50 \mathrm{~km} \mathrm{~s}^{-1}$, are seen in all $\mathrm{OH}$ profiles, except at Pos. 9.

Hydroxyl absorption of the near side of the EMR is seen at all positions at velocities around $-130 \mathrm{~km} \mathrm{~s}^{-1}$, with a $\Delta V_{\mathrm{FWHM}}$ of about $55 \mathrm{~km} \mathrm{~s}^{-1}$. At Pos. 4 and 6 the EMR profile widens as a result of blending with the absorption of the CND.

Strong absorption from the HNVG is seen at Pos. 5 and 6 at about $-170 \mathrm{~km} \mathrm{~s}^{-1}$, but is also seen weakly at all other positions except at Pos. 9. The $\Delta V_{\text {FWHM }}$ of the $+20 \mathrm{~km} \mathrm{~s}^{-1}$ cloud in $\mathrm{OH}$ absorption is estimated to be $42 \mathrm{~km} \mathrm{~s}^{-1}$ and a southward negative velocity gradient is observed between Pos. 7 and 9.

To investigate the existence of $\mathrm{OH}$ absorption in the blueshifted high-velocity cloud observed in $\mathrm{C}^{18} \mathrm{O} J=2-1$ emission by Genzel et al. (1990), we decomposed the $1667 \mathrm{MHz}$ $\mathrm{OH}$ profile at Pos. 3 by fitting nine Gaussians, see Fig. 3. In this figure we have labelled the Gaussian components found at this position, whose velocities correspond to well-known objects in the field of view, such as the HNVG, the EMR, the 3-kpc arm, the $-30 \mathrm{~km} \mathrm{~s}^{-1}$ arm, the Local/Sgr arm, and the $+50 \mathrm{~km} \mathrm{~s}^{-1}$ cloud. Furthermore, a wide-velocity feature (WVF) was found, centred at $-32 \mathrm{~km} \mathrm{~s}^{-1}$ with a $\Delta V_{\mathrm{FWHM}}$ of $107 \mathrm{~km} \mathrm{~s}^{-1}$, i.e. in 
OH 1667 MHz Gaussian fits at Pos. 3

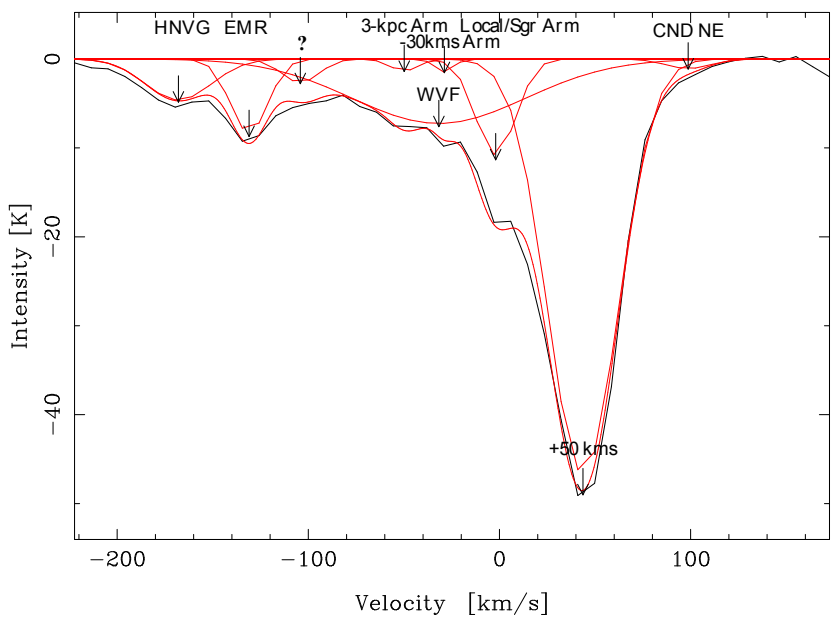

Fig. 3. Gaussian fits to the $\mathrm{OH} 1667 \mathrm{MHz}$ profile at Pos. 3. HNVG high negative velocity gas, EMR - expanding molecular ring, WVF wide velocity feature. (See Fig. 2c, for the corresponding $1665 \mathrm{MHz}$ profile.)

the same velocity regime as the blue-shifted high-velocity cloud. Two other features were also identified at Pos. 3, one centred at $-104 \mathrm{~km} \mathrm{~s}^{-1}$ with a $\Delta V_{\text {FWHM }}$ of $23 \mathrm{~km} \mathrm{~s}^{-1}$, and another one centred at $99 \mathrm{~km} \mathrm{~s}^{-1}$ with a $\Delta V_{\mathrm{FWHM}}$ of $28 \mathrm{~km} \mathrm{~s}^{-1}$. The $-104 \mathrm{~km} \mathrm{~s}^{-1}$ component is unknown, while the $99 \mathrm{~km} \mathrm{~s}^{-1}$ component seems to belong to the NE part of the CND, see Karlsson et al. (2003) and Karlsson (in prep.). The good agreement between the velocities from the Gaussian fit in Fig. 3, and established velocities in the literature for the respective features, leaves us with confidence on the parameters from the Gaussian fit.

A comparison of the VLA and Odin observations of the $+50 \mathrm{~km} \mathrm{~s}^{-1}$ cloud, the CND and the $+20 \mathrm{~km} \mathrm{~s}^{-1}$ cloud, can be seen in Figs. 4a-c, where the coloured areas show the $\mathrm{OH} 1667$ $\mathrm{MHz}$ absorption at an angular resolution of $7 " \times 5^{\prime \prime}$ as observed with the VLA (Karlsson et al. in prep.). The Odin beam at Pos. 1 is centred at the centroid of the $+50 \mathrm{~km} \mathrm{~s}^{-1}$ cloud, Pos. 5 is centred at Sgr $\mathrm{A}^{*}$ and covers the main parts of the CND, and Pos. 7 is centred at the $\mathrm{N}$ part of the $+20 \mathrm{~km} \mathrm{~s}^{-1}$ cloud. However, it should be noted that the $\mathrm{OH}$ absorption maps do not represent the full extent of the clouds, since the absorption intensity is dependent on the intensity of the continuum. Consequently, only the $\mathrm{W}$ part of the $+50 \mathrm{~km} \mathrm{~s}^{-1}$ cloud is seen at the single $50 \mathrm{~km} \mathrm{~s}^{-1}$ channel map in Fig. 4a, and only the northern part of the $+20 \mathrm{~km} \mathrm{~s}^{-1}$ cloud is seen at $24 \mathrm{~km} \mathrm{~s}^{-1}$ in Fig. 4c. The CND is displayed by the integration of two velocity channels, namely -73 and $103 \mathrm{~km} \mathrm{~s}^{-1}$ in Fig. 4b.

For the $+50 \mathrm{~km} \mathrm{~s}^{-1}$ cloud, the CND, the $+20 \mathrm{~km} \mathrm{~s}^{-1}$ cloud, and the EMR we have calculated the $\mathrm{OH}_{1667}$ optical depths and column densities. We have also estimated the $\mathrm{OH}_{1667}$ optical depths and column densities for the Local/Sgr arm, the $-30 \mathrm{~km} \mathrm{~s}^{-1}$ arm, and the 3-kpc arm, see Sect. 3.1.1.

\subsubsection{Optical depth and column density of $\mathrm{OH}_{1667}$}

The optical depth of $\mathrm{OH}$ absorption lines can be calculated by knowing the ratio of the main lines. This method eliminates the need for the standard assumption of placing all the continuum in the background with respect to the absorbing regions. The antenna temperature of an absorption line can be expressed as

$\Delta T_{\mathrm{A}}(v)=-T_{\mathrm{AC}} R\left[1-\mathrm{e}^{-\tau(v)}\right]$, where $T_{\mathrm{AC}}$ is the antenna temperature of the background continuum source, and $R$ is the fraction of the background source being covered by the molecular region, assumed to be equal to unity in this study. The difference in $T_{\mathrm{AC}}$ at 1665 and $1667 \mathrm{MHz}$ is negligible. We note that the absolute value of $\Delta T_{\mathrm{A}}(1665)$ is consistently less than and only sometimes nearly equal to the absolute value of $\Delta T_{\mathrm{A}}(1667)$. These circumstances justify our assumption that the $\mathrm{OH}$ excitation temperatures of 1665 and $1667 \mathrm{MHz}$ are equal, $T_{\mathrm{ex}}(1665)=T_{\mathrm{ex}}(1667)$ and, therefore that we can assume that $1<\Delta T_{\mathrm{A}}(1667) / \Delta T_{\mathrm{A}}(1665)<9 / 5$, corresponding to a variation of optical depth from high to low. The ratio of the 1667 and $1665 \mathrm{MHz}$ absorption lines therefore can be written as

$\frac{\Delta T_{\mathrm{A} \nu_{1}}}{\Delta T_{\mathrm{A} \nu_{2}}}=\frac{1-\mathrm{e}^{-\tau_{\nu_{1}}}}{1-\mathrm{e}^{-\left[\frac{v_{2} \times 5}{v_{1} \times 9}\right] \tau_{\nu_{1}}}}=\frac{1-\mathrm{e}^{-\tau_{\nu_{1}}}}{1-\mathrm{e}^{-0.5549 \times \tau_{\nu_{1}}}}$

where $v_{1}=1667.359 \mathrm{MHz}, v_{2}=1665.402 \mathrm{MHz}$, the relative line strengths are 9 and 5, respectively.

Since Eq. (2) cannot be solved analytically for the optical depth $\left(\tau_{1667}\right)$, a numerical solution is given in Fig. 5. For calculation of the $\tau_{1667}$ from the observed line ratio, we made a high-order polynomial fit to the curve in Fig. 5 using Matlab. Line ratio profiles were produced in NRAO AIPS and $\tau_{1667}$ as a function of radial velocity was calculated from the curve fit. The median ratio uncertainty was calculated to be 0.1 , and the average optical depth uncertainty was calculated to \pm 0.45 .

For calculation of the $\mathrm{OH}$ column densities we used the $1667 / 1665 \mathrm{MHz}$ ratio and numerically solved Eq. (2) for calculation of the optical depth at radial velocities and $\Delta V_{\mathrm{FWHM}} \mathrm{S}$ estimated by Gaussian fitting to the $1667 \mathrm{MHz} \mathrm{OH}$ absorption profiles. The optical depth for the $+50 \mathrm{~km} \mathrm{~s}^{-1}$ cloud in Table 4 is the most probable value, and we were not able to give an upper limit to the optical depth and column density there, since the $1667 / 1665 \mathrm{MHz}$ ratio approaches 1 , where the uncertainty of 0.1 is applied to the observed line ratio, and hence the optical depth goes towards infinity.

The OH 1667 MHz column densities were subsequently calculated from

$\frac{N_{\mathrm{OH}}}{T_{\mathrm{ex}}}=\frac{8 \pi k v_{1} \sum g_{\mathrm{i}}}{h c^{2} A_{22} g_{\mathrm{u}}} \int_{-\infty}^{+\infty}\langle\tau\rangle(v) \mathrm{d} v$

where $T_{\mathrm{ex}}$ is the excitation temperature, $k, h, c$ are the Boltzmann, and Planck constants and the speed of light, respectively. $A_{22}$ is the Einstein A-coefficient for the $1667 \mathrm{MHz} 2^{+}-2^{-}$ transition $\left(7.778 \times 10^{-11}\right), \sum g_{\mathrm{i}}$ is the sum of statistical weights, and $g_{\mathrm{u}}$ is the statistical weight of the upper level. The constant before the integral becomes $2.242 \times 10^{14}$, as the integral is converted from frequency to velocity. A relation between the excitation temperature and the hydrogen density, $n_{\mathrm{H}_{2}}$, for different kinetic temperatures is given by Goss (1968), and hence the $T_{\text {ex }}$ can be estimated once the kinetic temperature and hydrogen density are known.

The excitation temperature is, however, different for the observed regions. For the +50 and $+20 \mathrm{~km} \mathrm{~s}^{-1}$ clouds the average $n_{\mathrm{H}_{2}}$ is a few times $10^{4} \mathrm{~cm}^{-3}$ (Ferrière 2012), and we can perhaps assume that the 1665 and $1667 \mathrm{MHz} \mathrm{OH}$ transitions are thermalized (see Fig. 9 in Goss 1968) to the kinetic temperature of $80 \mathrm{~K}$ (Walmsley et al. 1986). However, the $\mathrm{OH}$ absorbing front sides of the clouds very likely are inhomogenous in density, temperature and abundance, and a lower limit to the kinetic temperature of $10-12 \mathrm{~K}$ appears from the analysis of our $\mathrm{C}^{18} \mathrm{O} J=2-1$ and 1-0 data (Sect. 3.5.1). Therefore it may be more reasonable to assume $T_{\mathrm{ex}}=40 \mathrm{~K}$. Since, according to Eq. (3), the average $\mathrm{OH}$ column density is linearly proportional to $T_{\mathrm{ex}}$ we then are 

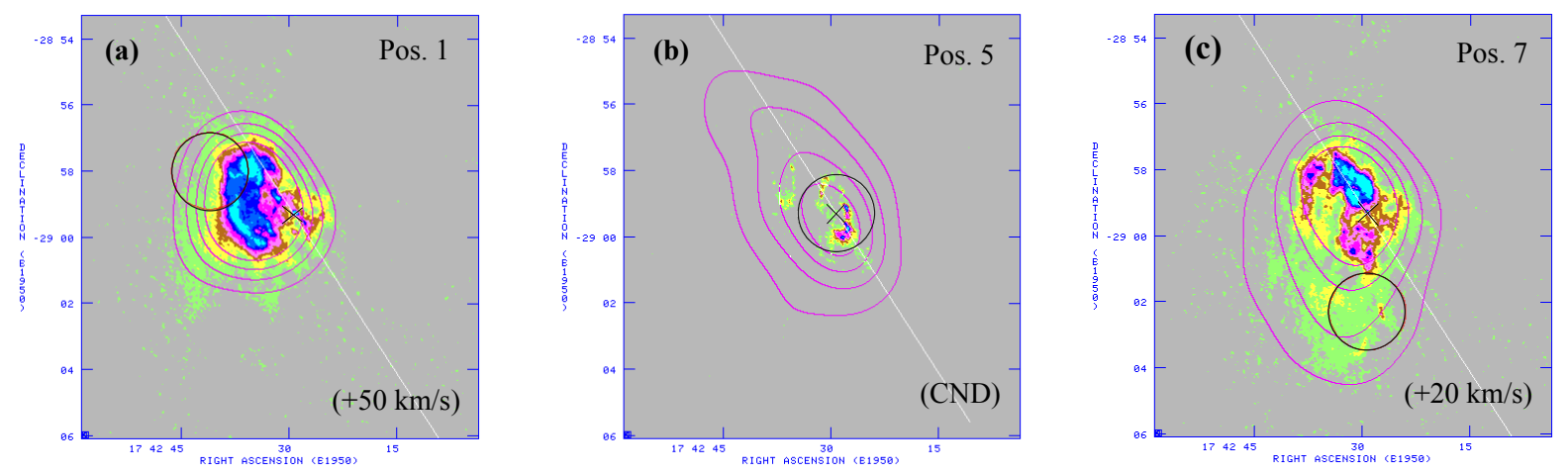

Fig. 4. Colour image of the $\mathrm{OH}$ absorption against a) the $50 \mathrm{~km} \mathrm{~s}^{-1}$ cloud at $50 \mathrm{~km} \mathrm{~s}^{-1}$, b) the CND at two channels integrated, namely -73 and $103 \mathrm{~km} \mathrm{~s}^{-1}$, and c) the $+20 \mathrm{~km} \mathrm{~s}^{-1}$ cloud at $24 \mathrm{~km} \mathrm{~s}^{-1}$ with $7^{\prime \prime} \times 5^{\prime \prime}$ resolution. Light blue colour indicates the deepest absorption. The contours delineate the $\mathrm{OH}$ absorption at $126^{\prime \prime}$ resolution, and the levels are 10, 20, 30, and $40 \mathrm{Jy} / \mathrm{beam}$. The circle indicates the Odin beam position and size, the black cross shows the position of Sgr A*, and the white diagonal line is parallell to the Galactic plane.

Table 4. $\mathrm{OH}_{1667}$ optical depths and column densities for the +50 and $+20 \mathrm{~km} \mathrm{~s}^{-1}$ clouds, the CND, the EMR, and the line-of-sight spiral arm features.

\begin{tabular}{lccccc}
\hline \hline Source & $\begin{array}{c}V_{\text {peak }} \\
\left(\mathrm{km} \mathrm{s}^{-1}\right)\end{array}$ & $\begin{array}{c}\Delta V_{\text {FWHM }} \\
\left(\mathrm{km} \mathrm{s}^{-1}\right)\end{array}$ & $\tau_{1667}$ & $\begin{array}{c}T_{\text {ex }} \\
(\mathrm{K})\end{array}$ & $\begin{array}{c}N(\mathrm{OH}) \\
\left(\mathrm{cm}^{-2}\right)\end{array}$ \\
\hline$+50 \mathrm{~km} \mathrm{~s}^{-1}$ & 49 & 31 & $4.3(+\mathrm{NA} /-1.4)$ & 40 & $1.2 \times 10^{18 a}$ \\
CND & 17 & 84 & $1.9(+0.4 /-0.3)$ & 80 & $2.9 \times 10^{18 a}$ \\
$+20 \mathrm{~km} \mathrm{~s}^{-1}$ & 23 & 42 & $3.3(+0.9 /-0.4)$ & 40 & $1.2 \times 10^{18 a}$ \\
EMR near side & -128 & 55 & $0.9(+0.4 /-0.3)$ & 30 & $3.3 \times 10^{17}(+1.5 /-1.1)$ \\
Local/Sgr arm & -6 & 16 & $1.1(+0.5 /-0.4)$ & 3 & $1.2 \times 10^{16}(+0.5 /-0.4)$ \\
$-30 \mathrm{~km} \mathrm{~s}^{-1}$ arm & -27 & 10 & $1.6(+0.7 /-0.5)$ & 3 & $1.1 \times 10^{16}(+0.4 /-0.4)$ \\
3-kpc arm & -54 & 13 & $1.6(+0.7 /-0.5)$ & 3 & $1.4 \times 10^{16}(+0.6 /-0.4)$ \\
\hline
\end{tabular}

Notes. ${ }^{(a)}$ Uncertainty is of the order of a factor 2, see Sect. 3.1.1.

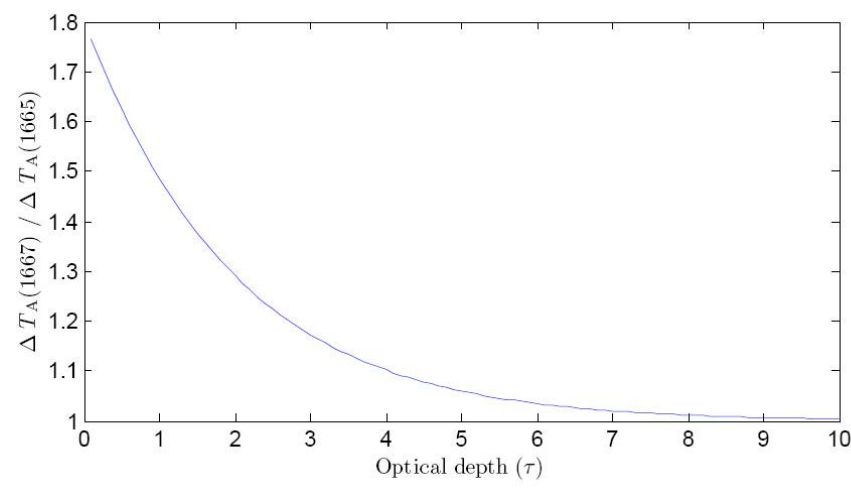

Fig. 5. $1667 \mathrm{MHz}$ OH optical depth versus the 1667/1665 MHz line ratio, calculated by Eq. (2).

tempted to believe that our column density estimates should be reliable to within a factor of two (up or down).

By smearing our $\mathrm{OH}$ observations to the $2.1^{\prime}$ resolution the entire CND is covered within the beam, see Fig. 4b. The physical environment in the CND is, however, quite different for the $\mathrm{NE}$ and SW regions and for the outer and inner parts, where the SW and inner parts are, due to their proximity to $\mathrm{Sgr} \mathrm{A}^{*}$, more exposed to the intense UV-field from the central star cluster and the SMBH, than the NE and outer parts. Furthermore, collisions between cloud clumps occur more frequently in the denser SW regions of the $\mathrm{CND}$, resulting in shock fronts that locally heat the gas further. We are therefore inclined to assume a higher $T_{\text {kin }}$ for the CND than for the +20 and $+50 \mathrm{~km} \mathrm{~s}^{-1}$ clouds. Tanaka et al. (2011) have studied the CND in detail, and find kinetic temperatures between 63 and $450 \mathrm{~K}$, and $n_{\mathrm{H}_{2}}=10^{4.1-7} \mathrm{~cm}^{-3}$, and assume $160 \mathrm{~K}$, and $n_{\mathrm{H}_{2}}=10^{6.5} \mathrm{~cm}^{-3}$ as a reasonable assumption in the CND. Ferrière (2012) made a comprehensive review of the CND and has arrived at the conclusion of adopting a value of $150 \mathrm{~K}$ for the CND. Our RADEX ${ }^{2}$ (van der Tak et al. 2007) analysis of the $\mathrm{H}_{2} \mathrm{O}$ red wing at the CND converges at a temperature of $160 \mathrm{~K}$. Although we may be tempted to use this high value as $T_{\text {ex }}$ in the CND, we again (as in the +50 and $+20 \mathrm{~km} \mathrm{~s}^{-1}$ clouds) consider the fact that the $\mathrm{OH}$ absorbing foreground gas most likely is inhomogenous. A lower limit to the kinetic temperature in the region of $13 \mathrm{~K}$ is found from our $\mathrm{C}^{18} \mathrm{O}$ analysis (Sect. 3.5.1). We therefore assume $T_{\mathrm{ex}}=80 \mathrm{~K}$, and again feel that our column density estimate should be accurate to within a factor of two (up or down).

For the EMR, the $\mathrm{OH}$ absorption profiles at all positions, except Pos. 5 and 6 which are contaminated by the CND, were averaged. Gaussians were fitted to the averaged $1667 \mathrm{MHz}$ absorption profile with the $\mathrm{XS}^{3}$ programme package, which is very convenient for fitting of multi-Gaussians to line profiles. The intensity ratio of $1667 / 1665 \mathrm{MHz}$ lines at the velocity of maximum absorption ( $\left.V_{\text {peak }}\right)$ and $\Delta V_{\text {FWHM }}$ was used for calculating the optical depth, and subsequently for calculation of the column density. For the EMR we adopt a value for $T_{\mathrm{ex}}$ of $30 \mathrm{~K}$, at $n_{\mathrm{H}_{2}}=10^{3-3.5} \mathrm{~cm}^{-3}$ (Tanaka et al. 2011). The resulting optical depths and column densities of the $+50 \mathrm{~km} \mathrm{~s}^{-1}$ cloud, the CND, the $+20 \mathrm{~km} \mathrm{~s}^{-1}$ cloud, and the EMR are summarized in Table 4 .

To enhance the signatures of the three line-of-sight spiral arm features, we averaged the profiles at Pos. 2, 3, 4, and 6,

\footnotetext{
2 http://www.sron.rug.nl/ vdtak/radex/radex.php

3 Developed by P. Bergman, Onsala Space Observatory.
} 

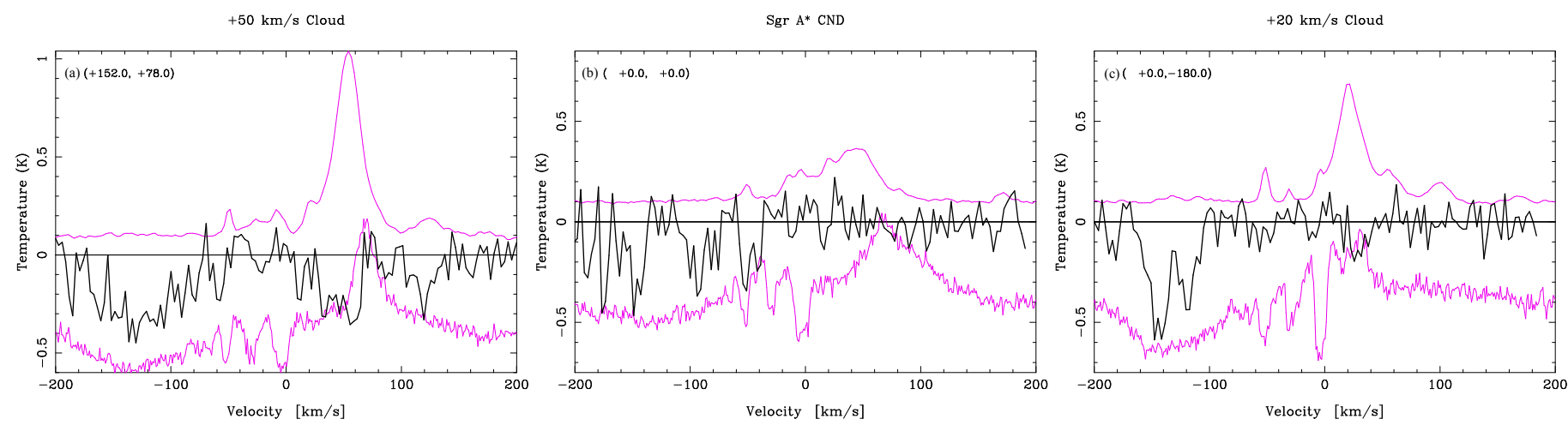

Fig. 6. $\mathrm{H}_{2}^{18} \mathrm{O}$ (thick black line), the $\mathrm{H}_{2} \mathrm{O}$ (thin magenta "noisy" line) and the $\mathrm{C}^{18} \mathrm{O}(1-0)$ (thin magenta "smooth" line) profiles towards a) the $+50 \mathrm{~km} \mathrm{~s}^{-1}$ cloud, b) the CND, and c) the $+20 \mathrm{~km} \mathrm{~s}^{-1}$ cloud. The $\mathrm{H}_{2}^{18} \mathrm{O}$ profiles have been multiplied by a factor of 10 and the channel resolution is $3.4 \mathrm{~km} \mathrm{~s}^{-1}$. The channel resolution of the $\mathrm{H}_{2} \mathrm{O}$ profiles is $1.0 \mathrm{~km} \mathrm{~s}^{-1}$, and the temperature scale has been reduced by $0.4 \mathrm{~K}$ for clarity. The temperature scale for $\mathrm{C}^{18} \mathrm{O}(1-0)$ has been increased by $0.1 \mathrm{~K}$, also for clarity.

where the signatures of these features are strongest in the 1665 and $1667 \mathrm{MHz}$ lines. For the line-of-sight spiral arm features we used a hydrogen density of $0.2 \mathrm{~cm}^{-3}$ (Burton 1988). The relation between the excitation temperature and the hydrogen density in the paper by Goss (1968), indicates that the excitation temperature converges at the cosmic background temperature of $3 \mathrm{~K}$ (rounded figure) at these low densities and kinetic temperatures of $100 \mathrm{~K}$ or less, which is in the expected range for the line-ofsight spiral arm features, and hence we use $T_{\mathrm{ex}}=3 \mathrm{~K}$ for those sources. The optical depths and column densities for the line-ofsight spiral arm features are shown in Table 4.

\subsection{Water $\left(\mathrm{H}_{2}^{16} \mathrm{O}\right.$ and $\left.\mathrm{H}_{2}^{18} \mathrm{O}\right)$}

The nine observed $\mathrm{H}_{2} \mathrm{O}$ profiles are presented in Fig. 2. Linear baselines have been subtracted and all the profiles have subsequently been boxcar-smoothed using three AOS channels which yields a final channel resolution of $1.0 \mathrm{~km} \mathrm{~s}^{-1}$. The intensity scales shown in Fig. 2 have not been corrected for the beam efficiency $(\approx 0.9)$. The original profiles all showed continuum levels which behaved qualitatively in agreement with the dust emission expected from an interpolation to the $\mathrm{H}_{2} \mathrm{O}$ frequency of the $800 \mu \mathrm{m}$ dust continuum map of Lis \& Carlstrom (1994), and $350 \mu \mathrm{m}$ map of Dowell et al. (1999), respectively (see Fig. 3 of Sandqvist et al. 2006). However, we do not judge the Odin position-switched continuum levels to be quantitatively reliable and have therefore chosen to estimate the Odin continuum temperatures $\left(T_{\text {cont }}\right)$ in a manner similar to Sandqvist et al. (2003). This method was based on the two above-mentioned continuum maps, and the values obtained are presented in Table 3.

A mixture of absorption and emission features are present in all of the $\mathrm{H}_{2} \mathrm{O}$ profiles, the absorptions dominating the negative velocities and the emissions dominating the positive velocities. In our discovery paper (Sandqvist et al. 2003) we used Gaussian fitting to identify four absorption features in the $\mathrm{Sgr}^{*} / \mathrm{CND}$ profile at $(0,0)$. These four absorption features are now seen to be present in all the nine positions observed. They originate in the spiral arm features that the line-of-sight to the Sgr A complex crosses: the Local/Sgr arm, the $-30 \mathrm{~km} \mathrm{~s}^{-1} \mathrm{arm}$, the 3-kpc arm, and the EMR. At Pos. 1, 2, and 3 there is strong $\mathrm{H}_{2} \mathrm{O}$ emission at velocities around $65 \mathrm{~km} \mathrm{~s}^{-1}$ which we attribute to the dissipation of shocks and turbulence where the Sgr A East shell meets the $+50 \mathrm{~km} \mathrm{~s}^{-1}$ cloud. By Gaussian fits to the profiles at Pos. 5, it was revealed that the component with velocity near
$70 \mathrm{~km} \mathrm{~s}^{-1}$ and the component with velocity near $-30 \mathrm{~km} \mathrm{~s}^{-1}$ (a broad feature, not to be confused with the narrow absorption feature near the same velocity), are both emission features originating in the $\mathrm{CND}$, the positive velocity component coming from the NE part of the CND, and the negative component is interpreted as coming from the SW part of the CND (Sandqvist et al. 2003). In the six profiles observed along the molecular belt (Pos. 1 to 7, except Pos. 5) the positive-velocity $\mathrm{H}_{2} \mathrm{O}$ emission component clearly traces out the well-known velocity gradient seen in many other molecular observations (e.g. Sandqvist 1989), ranging from $50 \mathrm{~km} \mathrm{~s}^{-1}$ in the NE to $10 \mathrm{~km} \mathrm{~s}^{-1}$ in the SW, see also Sect. 3.5.2, and Fig. 12.

The $\mathrm{H}_{2}^{18} \mathrm{O}$ profiles observed towards the $+50 \mathrm{~km} \mathrm{~s}^{-1}$ cloud, the CND and the $+20 \mathrm{~km} \mathrm{~s}^{-1}$ cloud are shown in Fig. 6, which also shows the corresponding $\mathrm{H}_{2} \mathrm{O}$ profiles. The $\mathrm{H}_{2}^{16} \mathrm{O}$ molecule and its much rarer isotopologue $\mathrm{H}_{2}^{18} \mathrm{O}$, trace parts of the cloud with different physical characteristics, which causes the velocity displacement between the two, see Fig. 6. Also displayed in Fig. 6 are the $\mathrm{C}^{18} \mathrm{O}(1-0)$ profiles obtained from the SEST survey of the Galactic centre (Lindqvist et al. 1995) by convolving the map data to the $2^{\prime} .1$-resolution of the Odin beam in the appropriate directions. The $\mathrm{H}_{2}^{18} \mathrm{O}$ profiles have been boxcarsmoothed using 9 AOS channels and simple second order baselines have been subtracted - note also that the $\mathrm{H}_{2}^{18} \mathrm{O}$ intensity scales have been multiplied by a factor of 10 . The observations were subsequently averaged, weighted by the system temperatures and integration times. The rms noise in all three $\mathrm{H}_{2}^{18} \mathrm{O}$ profiles is $7 \mathrm{mK}$. We have fitted Gaussians to all the absorption features we could reliably identify, the results of which are presented in Table 5.

A clear $\mathrm{H}_{2}^{18} \mathrm{O}$ absorption feature is seen in the profile observed towards the $+50 \mathrm{~km} \mathrm{~s}^{-1}$ cloud at a velocity of $46 \mathrm{~km} \mathrm{~s}^{-1}$. It agrees well with the velocity of the $\mathrm{C}^{18} \mathrm{O}$ feature, but the redward shift of the $\mathrm{H}_{2} \mathrm{O}$ peak is probably a result of strong self-absorption in this line at this position. Broad absorption is also seen from the near side of the EMR at $-130 \mathrm{~km} \mathrm{~s}^{-1}$, and even from the far side of the EMR at around $120 \mathrm{~km} \mathrm{~s}^{-1}$. In the CND profile there are a number of weak but identifiable $\mathrm{H}_{2}^{18} \mathrm{O}$ absorption features: the EMR near a velocity of $-150 \mathrm{~km} \mathrm{~s}^{-1}$, the SW region of the CND near $-90 \mathrm{~km} \mathrm{~s}^{-1}$, and the $3-\mathrm{kpc}$ arm near $-50 \mathrm{~km} \mathrm{~s}^{-1}$. The $+20 \mathrm{~km} \mathrm{~s}^{-1}$ cloud $\mathrm{H}_{2}^{18} \mathrm{O}$ profile shows absorption from the $+20 \mathrm{~km} \mathrm{~s}^{-1}$ cloud near a velocity of $29 \mathrm{~km} \mathrm{~s}^{-1}$, and a clear double absorption from the near side of the EMR at velocities near $-130 \mathrm{~km} \mathrm{~s}^{-1}$. 
Table 5. Water parameters from Gaussian fitting.

\begin{tabular}{llllllll}
\hline \hline Position & $\begin{array}{l}V \\
\left(\mathrm{~km} \mathrm{~s}^{-1}\right)\end{array}$ & $\begin{array}{l}\Delta V_{\mathrm{FWHM}} \\
\left(\mathrm{km} \mathrm{s}^{-1}\right)\end{array}$ & $\begin{array}{l}T_{\mathrm{A}}^{*} \\
(\mathrm{mK})\end{array}$ & $\tau_{\mathrm{o}}$ & $\begin{array}{l}N\left(\mathrm{H}_{2}^{18} \mathrm{O}\right) \\
\left(\mathrm{cm}^{-2}\right)\end{array}$ & $\begin{array}{l}N\left(\mathrm{H}_{2} \mathrm{O}\right)^{a} \\
\left(\mathrm{~cm}^{-2}\right)\end{array}$ & Source \\
\hline 1 & 120 & 20 & -23 & 0.12 & $1.1 \times 10^{13}$ & $2.8 \times 10^{15}$ & EMR (far side) \\
& 46 & 39 & -30 & 0.15 & $2.7 \times 10^{13}$ & $6.8 \times 10^{15}$ & $+50 \mathrm{~km} \mathrm{~s}^{-1} \mathrm{cloud}$ \\
& -126 & 73 & -36 & 0.19 & $6.3 \times 10^{13}$ & $1.6 \times 10^{16}$ & $\begin{array}{l}\text { EMR (near side) } \\
5\end{array}$ \\
& -50 & 16 & -25 & 0.21 & $1.6 \times 10^{13}$ & $4.0 \times 10^{15}$ & $3-\mathrm{kpc}$ arm \\
& -93 & 19 & -28 & 0.24 & $2.2 \times 10^{13}$ & $5.5 \times 10^{15}$ & CND \\
7 & -147 & 13 & -38 & 0.35 & $2.1 \times 10^{13}$ & $5.3 \times 10^{15}$ & EMR (near side) \\
& 29 & 15 & -17 & 0.09 & $0.6 \times 10^{13}$ & $1.6 \times 10^{15}$ & $+20 \mathrm{~km} \mathrm{~s}$ cloud \\
& -119 & 14 & -42 & 0.24 & $1.6 \times 10^{13}$ & $4.0 \times 10^{15}$ & EMR (near side) \\
Average of Pos. 2, 3, 4, and $6^{b}$ & -143 & 19 & -57 & 0.34 & $3.0 \times 10^{13}$ & $7.5 \times 10^{15}$ & EMR (near side) \\
\hline
\end{tabular}

Notes. ${ }^{(a)}$ Assuming $\mathrm{H}_{2} \mathrm{O} / \mathrm{H}_{2}^{18} \mathrm{O}=250$ (Wilson \& Rood 1994). ${ }^{(b)}$ These values refer to $\mathrm{H}_{2} \mathrm{O}$, see text. ${ }^{(c)}$ Calculated from observations of $\mathrm{H}_{2} \mathrm{O}$.

In order to determine the column densities $N\left(\mathrm{o}-\mathrm{H}_{2} \mathrm{O}\right)$ and $N\left(\mathrm{o}-\mathrm{H}_{2}^{18} \mathrm{O}\right)$ we follow the "curve of growth" analysis used by Plume et al. (2004), where the lower state column density is given by

$N_{l}=\frac{g_{1}}{g_{\mathrm{u}}} \frac{8 \pi \sqrt{\pi}}{\lambda_{0}^{3} A_{\mathrm{ul}}} \tau_{0} \frac{\Delta V_{\mathrm{FWHM}}}{2 \sqrt{\ln 2}}$

where $\lambda_{0}\left(\mathrm{o}-\mathrm{H}_{2}^{18} \mathrm{O}\right)=547.39 \mu \mathrm{m}, \lambda_{0}\left(\mathrm{o}-\mathrm{H}_{2} \mathrm{O}\right)=538.66 \mu \mathrm{m}, A_{\mathrm{ul}}=$ $3.5 \times 10^{-3} \mathrm{~s}^{-1}$ (for both $\mathrm{H}_{2}^{18} \mathrm{O}$ and $\mathrm{H}_{2} \mathrm{O}$ ), and $g_{1} / g_{u}=1$.

Equation (4) is based upon the assumption that $T_{\mathrm{ex}} \ll h v / k=$ $27 \mathrm{~K}$, which is very likely to be the case since the actual cloud densities $\left(10^{3}-10^{6.5} \mathrm{~cm}^{-3}\right)$ are much lower than the critical density of these water lines, $3 \times 10^{8} \mathrm{~cm}^{-3}$ (e.g. Hjalmarson et al. 2003). This also implies that the dominant part of the molecular population is residing in lower state and hence Eq. (4) provides a first order approximation of the total column densities. Inserting numerical values of the constants in Eq. (4) we arrive at the following estimates of the total column densities

$N\left(\mathrm{H}_{2} \mathrm{O}\right)=4.89 \times 10^{12} \tau_{\mathrm{o}}\left(\mathrm{H}_{2} \mathrm{O}\right) \Delta V_{\text {FWHM }}$

$N\left(\mathrm{H}_{2}^{18} \mathrm{O}\right)=4.66 \times 10^{12} \tau_{\mathrm{o}}\left(\mathrm{H}_{2}^{18} \mathrm{O}\right) \Delta V_{\mathrm{FWHM}}$

where $\tau_{\mathrm{o}}$ is the central optical depth of the Gaussian fitted to the absorption feature and is given by

$\tau_{\mathrm{o}}=-\ln \left[1+\frac{T_{\mathrm{A}}^{*}}{T_{\mathrm{cont}}}\right]$.

Here $T_{\mathrm{A}}^{*}$ is the antenna temperature of the absorption feature (from the Gaussian fit, see Table 5) and is a negative number, and $T_{\text {cont }}$ is the continuum background temperature - see Table 3 . The optical depths and column densities of the $\mathrm{H}_{2}^{18} \mathrm{O}$ absorption features, calculated from Eqs. (7) and (6) are given in Table 5. By adopting an $\mathrm{H}_{2} \mathrm{O} / \mathrm{H}_{2}^{18} \mathrm{O}$ ratio of 250 for the $\mathrm{GC}$, as suggested by Wilson \& Rood (1994), we have also calculated the column densities of $\mathrm{H}_{2} \mathrm{O}$ shown in Table 5 .

It is well-known that the $557 \mathrm{GHz} \mathrm{H}_{2} \mathrm{O}$ line has a very high optical depth and in most cases the $\mathrm{H}_{2} \mathrm{O}$ absorption lines are saturated (e.g. Plume et al. 2004). This is certainly also true for most of the absorption features which we observe towards the Sgr A complex. It is thus generally not possible to directly determine $\mathrm{H}_{2} \mathrm{O}$ column densities for these features. However, there is one exception, namely the absorption from the $-30 \mathrm{~km} \mathrm{~s}^{-1} \mathrm{arm}$. To illustrate this, we consider the average $\mathrm{H}_{2} \mathrm{O}$ profile of Pos. 2 , 3,4 , and 6 shown in Fig. 7. The $-130,-50$, and $0 \mathrm{~km} \mathrm{~s}^{-1}$ absorption features all have the same antenna temperature, namely

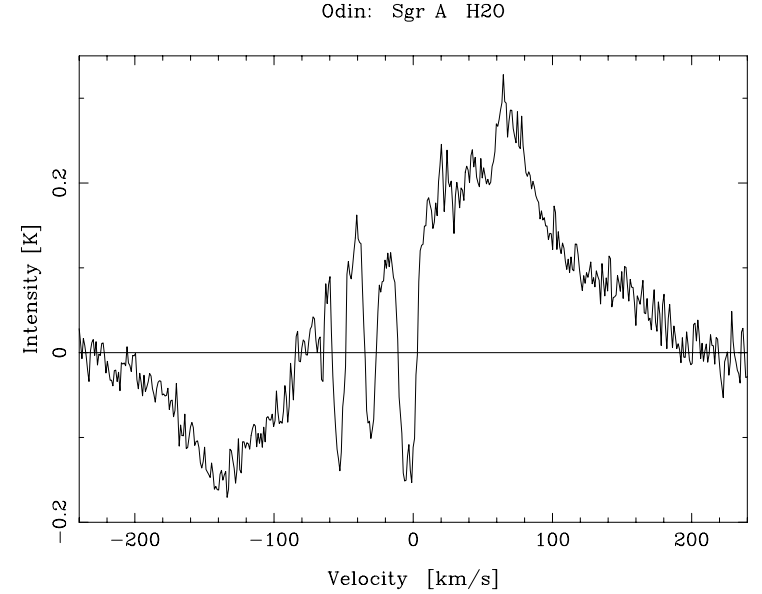

Fig. 7. Average $\mathrm{H}_{2} \mathrm{O}$ profile of Pos. 2, 3, 4, and 6. The channel resolution is $1.0 \mathrm{~km} \mathrm{~s}^{-1}$.

$-150 \mathrm{mK}$. We shall assume that they are all saturated and that this is the value of the background continuum. This value is in good agreement with the average of $155 \mathrm{mK}$ of the $T_{\text {cont }}$ for the four relevant positions (see Table 3), thus supporting the reality of our estimated $T_{\text {cont }}$ values listed in this table. A Gaussian fit yields a $T_{\mathrm{A}}^{*}$ absorption of $-230 \mathrm{mK}$ for the $-30 \mathrm{~km} \mathrm{~s}^{-1}$ arm in Fig. 7. As seen in this figure, the narrow absorption takes place against a wider background $\mathrm{H}_{2} \mathrm{O}$ emission profile which contributes an additional antenna temperature of $130 \mathrm{mK}$ to the background continuum, thus yielding an effective $T_{\text {cont }}$ value of $280 \mathrm{mK}$. Using Eq. (7) we then obtain an optical depth $\tau_{o}$ of 1.7, and with a Gaussian value of $\Delta V_{\mathrm{FWHM}}=7 \mathrm{~km} \mathrm{~s}^{-1}$, we get an $\mathrm{H}_{2} \mathrm{O}$ column density of $5.9 \times 10^{13} \mathrm{~cm}^{-2}$ from Eq. (5), see Table 5 .

\subsection{Ammonia $\left(\mathrm{NH}_{3}\right)$}

Unfortunately, the submm receiver tuned for the $572 \mathrm{GHz}$ $\mathrm{NH}_{3}$ line was unstable in frequency for a large part of the observing sessions and no data were obtained for the observations of $\mathrm{NH}_{3}$ towards the +50 and $+20 \mathrm{~km} \mathrm{~s}^{-1}$ clouds and the CND. However, limited success was achieved for the four Sgr A East rim positions $(2,3,4$, and 6$)$. Integrating these four positions together, weak $\mathrm{NH}_{3}$ absorption features were detected from the Local/Sgr arm, the $-30 \mathrm{~km} \mathrm{~s}^{-1}$ arm and the 3-kpc arm, at velocities near $0,-30$, and $-50 \mathrm{~km} \mathrm{~s}^{-1}$, respectively (see Fig. 8). 
Table 6. $\mathrm{NH}_{3}$ optical depths and column densities in the spiral arm features.

\begin{tabular}{lccccc}
\hline \hline Source & $\begin{array}{c}V \\
\left(\mathrm{~km} \mathrm{~s}^{-1}\right)\end{array}$ & $\begin{array}{c}\Delta V_{\mathrm{FWHM}} \\
\left(\mathrm{km} \mathrm{s}^{-1}\right)\end{array}$ & $\begin{array}{c}T_{\mathrm{A}}^{*} \\
(\mathrm{mK})\end{array}$ & $\tau_{o}$ & $\begin{array}{c}N\left(\mathrm{NH}_{3}\right) \\
\left(\mathrm{cm}^{-2}\right)\end{array}$ \\
\hline Local/Sgr arm & -5 & 12 & -54 & 0.41 & $1.8 \times 10^{13}$ \\
$-30 \mathrm{~km} \mathrm{~s}{ }^{-1}$ arm & -27 & 8 & -46 & 0.34 & $1.0 \times 10^{13}$ \\
3-kpc arm & -51 & 5 & -74 & 0.62 & $1.2 \times 10^{13}$ \\
\hline
\end{tabular}

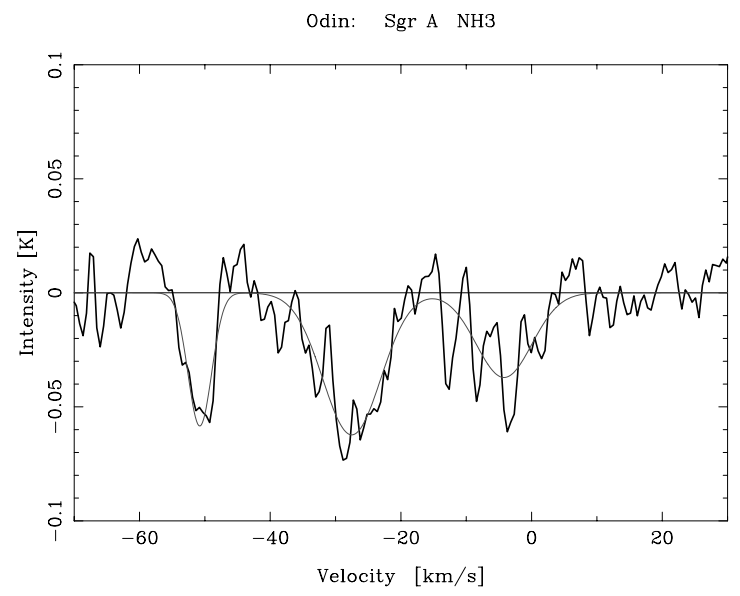

Fig. 8. $\mathrm{NH}_{3}$ profile obtained by averaging the four Sgr A East rim positions (Positions 2, 3, 4, and 6). Gaussian fits have been applied to the three absorption components. The channel resolution is $0.5 \mathrm{~km} \mathrm{~s}^{-1}$. The Local/Sgr arm, $-30 \mathrm{~km} \mathrm{~s}^{-1}$ arm and the $3-\mathrm{kpc}$ arm can be identified in the figure.

The column density of ortho- $\mathrm{NH}_{3}$ for these three components can be determined in a manner similar to the $\mathrm{H}_{2}^{18} \mathrm{O}$ above and that of Wirström et al. (2010):

$N\left(N H_{3}\right)=3.69 \times 10^{12} \tau_{\mathrm{o}}\left(N H_{3}\right) \Delta V_{\text {FWHM }}$.

This relation is valid under the same assumption as the previous Eqs. (4)-(6), namely that the relevant cloud densities are much lower than the critical density of the $\mathrm{NH}_{3}$ line in question $(5 \times$ $10^{7} \mathrm{~cm}^{-3}$; e.g. Hjalmarson et al. 2003). The results are given in Table 6.

\subsection{Atomic carbon and carbon monoxide $(\mathrm{Cl}$ and $\mathrm{CO} J=5-4$ )}

According to Phillips \& Huggins (1981), the total column density of an optically thin $\mathrm{C}_{\mathrm{I}}$ line can be calculated by means of the subsequent Eqs. (9) and (10).

$N_{\mathrm{CI}}=\frac{8 \pi k v^{2} \Delta V T_{\mathrm{A}}^{*}}{3 h c^{3} A_{10} \mathrm{e}^{-E_{1} / k T_{\mathrm{ex}}}} f$

where

$f=1+3 \mathrm{e}^{-E_{1} / k T_{\text {ex }}}+5 \mathrm{e}^{-E_{2} / k T_{\text {ex }}}$

where $\Delta V$ is the full velocity width at half maximum of the line, $A_{10}$ the Einstein A-coefficient of the $\mathrm{C}_{\mathrm{I}}\left({ }^{3} \mathrm{P}_{1}-{ }^{3} \mathrm{P}_{0}\right)$ transition $\left(8 \times 10^{-8} \mathrm{~s}\right), E_{1} \approx 23.5 \mathrm{~K}, E_{2} \approx 62.5 \mathrm{~K}$. Noting that $h v / k \sim 24 \mathrm{~K}$ for populating the ${ }^{3} \mathrm{P}_{1}$ level of $\mathrm{C}_{\mathrm{I}}, T_{\mathrm{ex}}$ is taken as $24 \mathrm{~K}$ (Phillips \& Huggins 1981). For C I Martin et al. (2004) find $T_{\mathrm{A}}^{*}$ approximately $6.5 \mathrm{~K}$ in the $+50 \mathrm{~km} \mathrm{~s}^{-1}$ cloud, $4 \mathrm{~K}$ in the $\mathrm{CND}$, and $2.5 \mathrm{~K}$ in the $+20 \mathrm{~km} \mathrm{~s}^{-1}$ cloud, which agrees reasonably well with our results. However, it is likely that the $\mathrm{C}_{\mathrm{I}}$ line is optically thick in
Table 7. C I column densities for the $+50 \mathrm{~km} \mathrm{~s}^{-1}$ cloud, the CND, and the $+20 \mathrm{~km} \mathrm{~s}^{-1}$ cloud.

\begin{tabular}{lcccc}
\hline \hline Source & $\begin{array}{c}V \\
\left(\mathrm{~km} \mathrm{~s}^{-1}\right)\end{array}$ & $\begin{array}{c}\Delta V_{\mathrm{FWHM}} \\
\left(\mathrm{km} \mathrm{s}^{-1}\right)\end{array}$ & $\begin{array}{c}T_{\mathrm{A}}^{*} \\
(\mathrm{~K})\end{array}$ & $\begin{array}{c}N\left(\mathrm{C}_{\mathrm{I}}\right) \\
\left(\mathrm{cm}^{-2}\right)\end{array}$ \\
\hline$+50 \mathrm{~km} \mathrm{~s}^{-1} \mathrm{Cl}$. & 57 & 34 & 7.5 & $\geq 3.3 \times 10^{18}$ \\
$\mathrm{CND}$ & 53 & 35 & 4.9 & $\geq 2.3 \times 10^{18}$ \\
$+20 \mathrm{~km} \mathrm{~s}^{-1} \mathrm{Cl}$. & 22 & 29 & 3.1 & $\geq 1.2 \times 10^{18}$ \\
\hline
\end{tabular}

dense molecular clouds, and the optical depths may be as high as 10 (Phillips \& Huggins 1981). Our assumption of an optically thin line therefore may not be justified. However, in the lack of calculation procedures for optically thick $\mathrm{C}_{\mathrm{I}}$ lines, we consider the $N\left(\mathrm{C}_{\mathrm{I}}\right)$ in the main sources in Table 7, as lower limits.

The Odin observations of the $576 \mathrm{GHz} J=5-4 \mathrm{CO}$ and $492 \mathrm{GHz} \mathrm{C}_{\mathrm{I}}$ lines towards the $+50 \mathrm{~km} \mathrm{~s}^{-1}$ cloud, the CND and the $+20 \mathrm{~km} \mathrm{~s}^{-1}$ cloud are shown in Fig. 9. Here they are compared with the goundbased South Pole observations with the Antarctic Submillimetre Telescope and Remote Observatory (AST/RO) of the $807 \mathrm{GHz} J=7-6$ and $461 \mathrm{GHz} J=4-3$ CO lines (Martin et al. 2004), where the mapping data have been smoothed to the Odin 2 -arcmin resolution. The pronounced absorption features seen in the $\mathrm{H}_{2} \mathrm{O}$ line near $0,-30$ and $-50 \mathrm{~km} \mathrm{~s}^{-1}$ are not seen in the Odin CO $J=5-4$ line, but only in the lower excitation $\mathrm{CO} J=4-3$ line, due to the low excitation temperatures in the spiral arms. It is interesting that similar infrared absorption features arising from cold $\mathrm{H}_{3}^{+}$have also been detected by Oka et al. (2005). We also note double-peaked emission between 20 and $60 \mathrm{~km} \mathrm{~s}^{-1}$ in different transitions at the three main positions, see Fig. 9.

\subsection{Carbon monoxide $\left(C^{18} O \mathrm{~J}=2-1\right.$ and $\left.\mathrm{J}=1-0\right)$}

In Fig. A.1 in Appendix A, we present the observed $\mathrm{C}^{18} \mathrm{O}$ $J=2-1$ profiles plotted on a Galactic coordinate offset grid; the temperature scale has not been corrected for the beam efficiency. Only linear baselines have been subtracted. The emission profiles are gridded on an $(l, b)$ plane, the velocity range is -200 to $200 \mathrm{~km} \mathrm{~s}^{-1}$, and the antenna temperature ranges from -100 to $-1700 \mathrm{mK}$. The velocity resolution is $1.0 \mathrm{~km} \mathrm{~s}^{-1}$.

\subsection{1. $\mathrm{C}^{18} \mathrm{O}$ excitation temperatures and column densities}

For comparison between the $\mathrm{C}^{18} \mathrm{O} J=2-1$ and $\mathrm{C}^{18} \mathrm{O} J=1-0$ emissions at the three main positions, we have included the $J=1-0$ emission spectra of Lindqvist et al. (1995) in our Fig. 10. The emission ratios of $\mathrm{C}^{18} \mathrm{O} J=2-1$ and $\mathrm{C}^{18} \mathrm{O} J=1-0$ were used for estimation of $T_{\text {rot }}$ by means of Eq. (11) (Goldsmith 1999).

$\ln \left[\int T_{\mathrm{mb}} \mathrm{d} v \frac{8 \pi \times 10^{5} k v^{2}}{h c^{3} g_{\mathrm{ul}} A_{\mathrm{ul}}}\right]=\ln \frac{N}{Z}-\frac{E_{\mathrm{u}}}{k} \frac{1}{T_{\mathrm{rot}}}$.

Plotting this equation allows us to determine $T_{\text {rot }}$ from the slope of the line $\left(-1 / T_{\text {rot }}\right)$, and $\ln (N / Z)$ from the line intercept on the $y$-axis. In Eq. (11), $\int T_{\mathrm{mb}} \mathrm{d} v$ is the integrated main beam intensity from observations, $A_{\mathrm{ul}}$ denotes the Einstein A-coefficient for the transition, $\mathrm{d} v$ is the velocity interval, and $v$ is the frequency in $\mathrm{GHz}$ for the transition.

We used $v_{10}=109.7821734 \mathrm{GHz}, A_{10}=6.266 \times 10^{-8}$, $g_{10}=3, E_{1} / k=5.27 \mathrm{~K}$ for the $J=1-0$ transition, and $v_{21}=$ $219.5603541 \mathrm{GHz}, A_{21}=6.011 \times 10^{-7}, g_{21}=5$ and $E_{2} / k=$ $15.81 \mathrm{~K}$, for the $J=2-1$ transition (Winnewisser et al. 1985). 
R. Karlsson et al.: $\mathrm{OH}, \mathrm{H}_{2} \mathrm{O}, \mathrm{CO}, \mathrm{NH}_{3}$, and $\mathrm{C} \mathrm{I}$ in the Sgr A complex

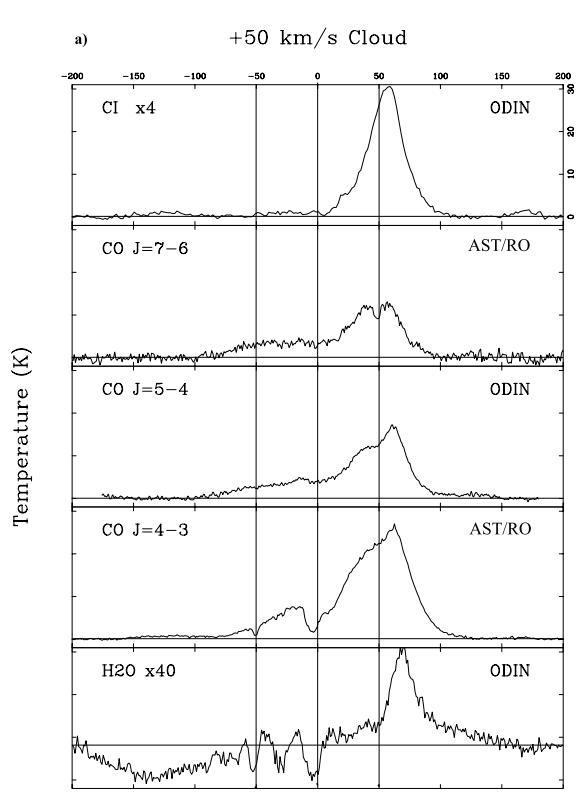

Velocity $(\mathrm{km} / \mathrm{s})$

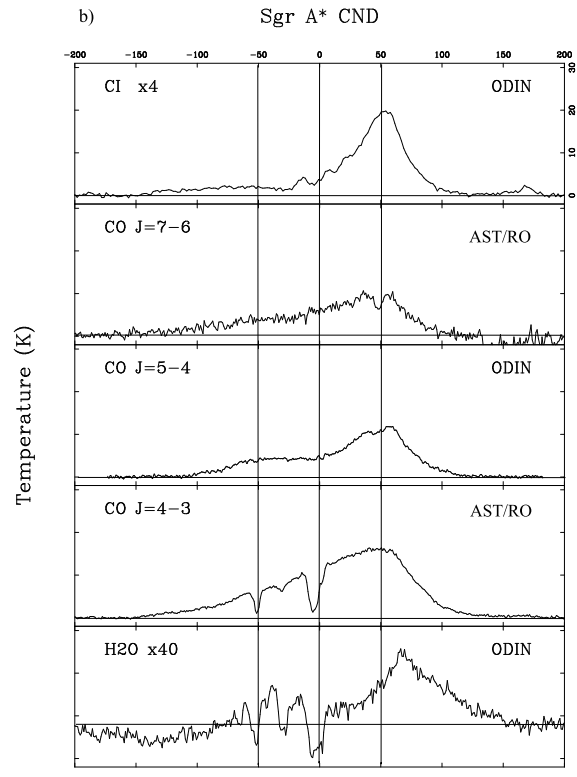

Velocity $(\mathrm{km} / \mathrm{s})$

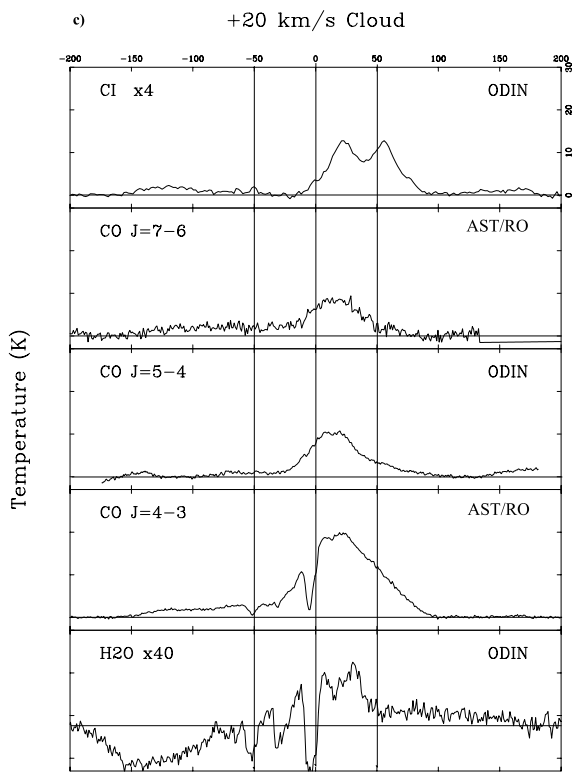

Velocity $(\mathrm{km} / \mathrm{s})$

Fig. 9. Odin $\mathrm{C}_{\mathrm{I}}$, $\mathrm{CO} J=5-4$, and $\mathrm{H}_{2} \mathrm{O}$ profiles towards a) the $+50 \mathrm{~km} \mathrm{~s}^{-1}$ cloud, b) the CND, and c) the $+20 \mathrm{~km} \mathrm{~s}^{-1}$ cloud. The $\mathrm{H}_{2} \mathrm{O}$ zero-line is nominally drawn at the $8 \mathrm{~K}$ level. Note that the $\mathrm{H}_{2} \mathrm{O}$ and $\mathrm{C}_{\mathrm{I}}$ intensities have been multiplied by factors of 40 and 4 , respectively. Odin-beamsmoothed ground-based observations of the $J=4-3$ and $J=7-6$ transitions of CO (Martin et al. 2004), are included for comparison. The velocity scale $\left(-200\right.$ to $200 \mathrm{~km} \mathrm{~s}^{-1}$ ) is shown on top of the diagrams, and the temperature scale ( -2 to $\left.31 \mathrm{~K}\right)$ is on the top right side in each diagram.

a)

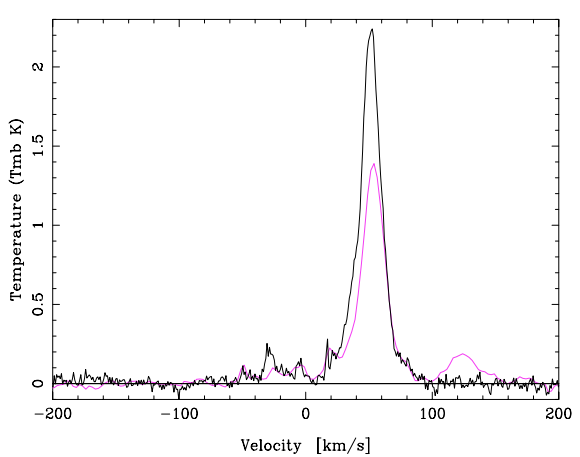

b)

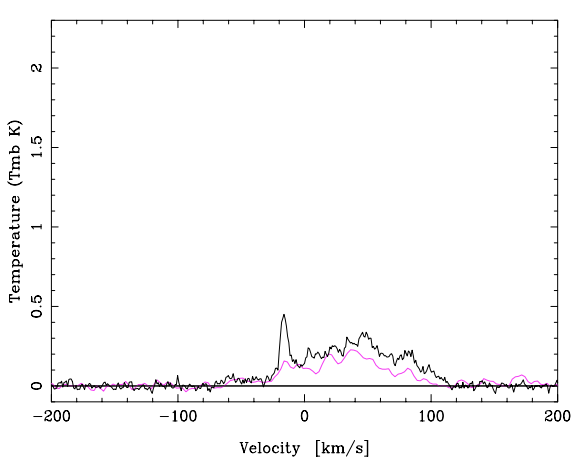

c)

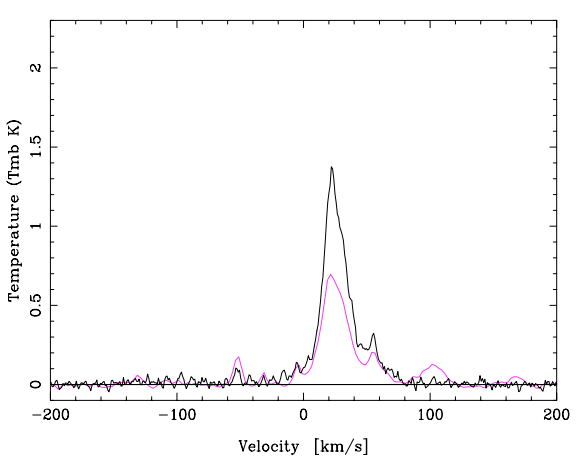

Fig. 10. $\mathrm{C}^{18} \mathrm{O} J=1-0$ (red smooth) and the $\mathrm{C}^{18} \mathrm{O} J=2-1$ (black noisy) emission towards a) the $\left.+50 \mathrm{~km} \mathrm{~s}^{-1} \mathrm{cloud,} \mathbf{b}\right)$ the $\mathrm{CND}$, and $\mathbf{c}$ ) the $+20 \mathrm{~km} \mathrm{~s}^{-1}$ cloud. The channel resolution is $3 \mathrm{~km} \mathrm{~s}^{-1}$ and $1 \mathrm{~km} \mathrm{~s}^{-1}$, for the $J=1-0$ and $J=2-1$ lines, respectively.

Table 8. Excitation temperatures and column densities for $\mathrm{C}^{18} \mathrm{O}$.

\begin{tabular}{lcccccccc}
\hline \hline Source & $\begin{array}{c}T_{\mathrm{mb} 10} \\
(\mathrm{~K})\end{array}$ & $\begin{array}{c}T_{\mathrm{mb} 21} \\
(\mathrm{~K})\end{array}$ & $\begin{array}{c}\int_{\left(\mathrm{K} \mathrm{km} \mathrm{s}^{-1}\right)} T_{\mathrm{mb10}} \mathrm{d} v \\
\left(\begin{array}{ll}\left.\mathrm{K} \mathrm{km} \mathrm{s}^{-1}\right) \\
T_{\mathrm{mb21}} \mathrm{d} v\end{array}\right.\end{array}$ & $\begin{array}{c}T_{\text {rot }} \\
(\mathrm{K})\end{array}$ & $\mathrm{Z}^{a}$ & $\begin{array}{c}N\left(\mathrm{C}^{18} \mathrm{O}\right) \\
\left(\mathrm{cm}^{-2}\right)\end{array}$ & $\begin{array}{c}N\left(\mathrm{H}_{2}\right) \\
\left(\mathrm{cm}^{-2}\right)\end{array}$ \\
\hline$+50 \mathrm{~km} \mathrm{~s}^{-1}$ & 1.38 & 2.22 & 36.5 & 51.6 & 10.2 & 4.22 & $3.2 \times 10^{16}$ & $1.6 \times 10^{23}$ \\
CND & 0.22 & 0.32 & 16.6 & 28.6 & 12.7 & 5.20 & $1.6 \times 10^{16}$ & $0.8 \times 10^{23}$ \\
$+20 \mathrm{~km} \mathrm{~s}^{-1}$ & 0.68 & 1.37 & 21.2 & 36.1 & 12.4 & 5.08 & $2.1 \times 10^{16}$ & $1.0 \times 10^{23}$ \\
\hline
\end{tabular}

Notes. ${ }^{(a)}$ Winnewisser et al. (1985), http://spec.jpl.nasa.gov/ftp/pub/catalog/catdir.cat

Subsequent calculations give $N\left(\mathrm{C}^{18} \mathrm{O}\right)$ and $N\left(\mathrm{H}_{2}\right)$, assuming a $\mathrm{C}^{18} \mathrm{O} / \mathrm{H}_{2}$ abundance ratio of $2 \times 10^{-7}$ (Goldsmith 1999). Our results for the three main positions are given in Table 8 .

The low $\mathrm{C}^{18} \mathrm{O}$ population distribution temperatures of 10-13 $\mathrm{K}$ determined for the $+50 \mathrm{~km} \mathrm{~s}^{-1}$ cloud, the CND and the $+20 \mathrm{~km} \mathrm{~s}^{-1}$ cloud from our $J=2-1$ and $J=1-0$ observations, are strong measures of low excitation conditions (low average cloud temperatures at densities of $10^{4} \mathrm{~cm}^{-3}$ or higher, and correspondingly higher temperatures if the densities are lower). Since the critical density for excitation of the $557 \mathrm{GHz}$ o- $\mathrm{H}_{2} \mathrm{O}$ and $548 \mathrm{GHz} 0-\mathrm{H}_{2}^{18} \mathrm{O}$ ground state rotational transitions is as high as $5 \times 10^{8} \mathrm{~cm}^{-3}$ and that for excitation of the $572 \mathrm{GHz}$ o- $\mathrm{NH}_{3}$ ground state rotational transition is $5 \times 10^{7} \mathrm{~cm}^{-3}$ (e.g. Hjalmarson et al. 2003), we arrive at the conclusion that these lines must be very poorly excited in the relevant absorption regions. This means that the excitation temperatures will be very 

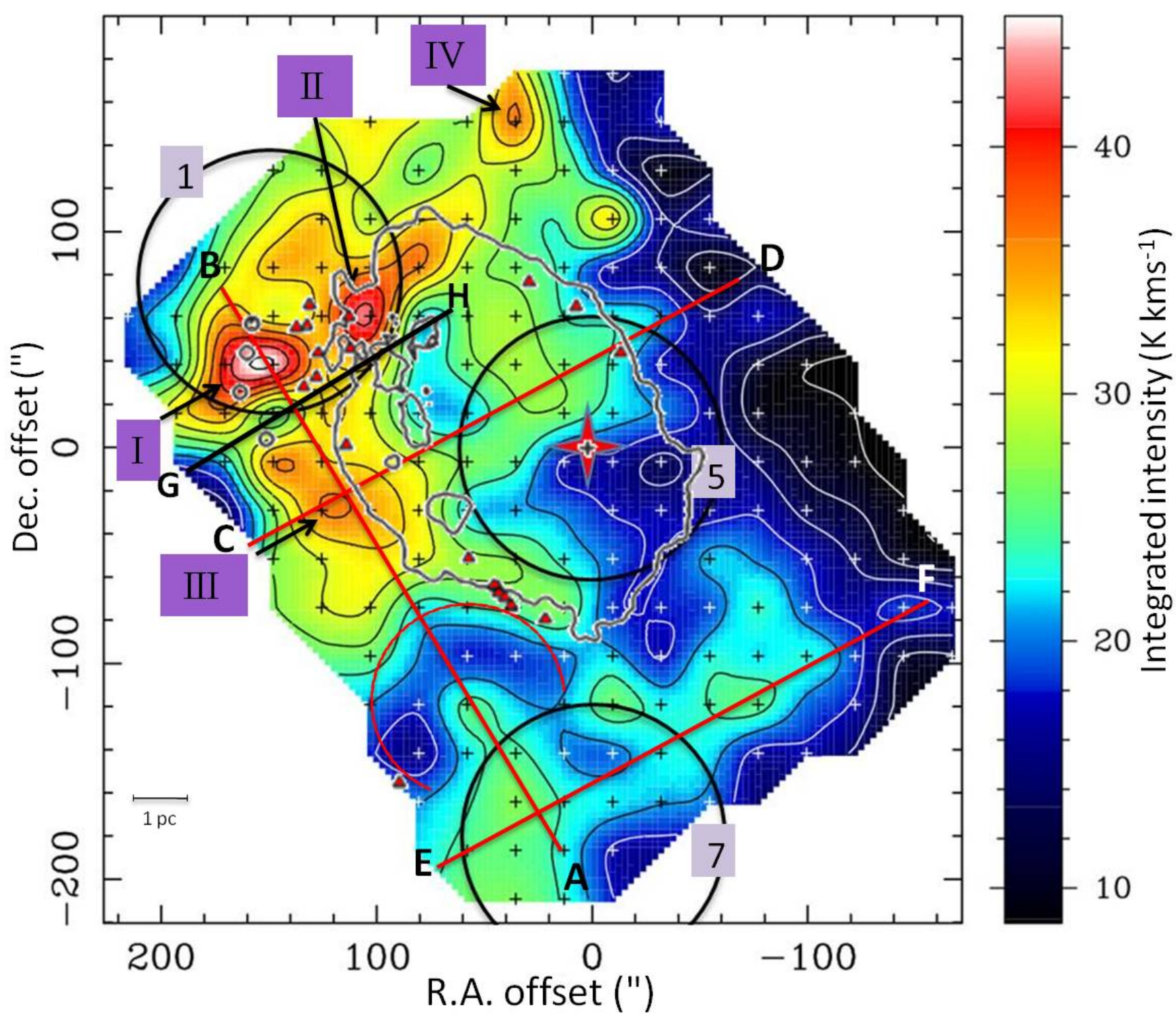

Fig. 11. Total integrated intensity ( -200 to $200 \mathrm{~km} \mathrm{~s}^{-1}$ ) of antenna temperature of SEST observations of $\mathrm{C}^{18} \mathrm{O} J=2-1$ towards the Sgr A complex. Sgr A* is marked with a red star, and the large circles mark the main positions of the $+50 \mathrm{~km} \mathrm{~s}^{-1}$ cloud (1), the CND (5), and the $+20 \mathrm{~km} \mathrm{~s}{ }^{-1}$ cloud (7). The red lines and letters indicate the directions of position-velocity diagrams discussed in Sects. 3.5.2, and 3.5.3, and the Roman numerals refer to objects discussed in the same sections. The black line $\mathrm{G}-\mathrm{H}$ indicates the position of the velocity cut shown in Fig. 14 . The grey contour line delineates the Sgr A East $18-\mathrm{cm}$ continuum emission at $0.15 \mathrm{Jy} /$ beam (Karlsson et al., in prep.). The small grey circles, near Component I, indicate the positions of the four compact $\mathrm{H}$ II regions (Ekers et al. 1983). The red triangles indicate positions of $1720 \mathrm{MHz} \mathrm{OH}$ Supernova Remnant (SNR) masers observed by Yusef-Zadeh et al. (1996, 2001), Karlsson et al. (2003), and Sjouwerman \& Pihlström (2008). The red arc line indicates the expanding shell associated with the SNR (G359.92-0.09). Offsets in the map are in arcseconds in the equatorial coordinate system, and relative to the position of Sgr A*. The HPBW is $24^{\prime \prime}$.

low, and that the molecular populations will reside essentially only in the lowest energy states, in which case Eqs. (5), (6) and (8) should be good measures of the total column densities. These logical conclusions are consistent with RADEX modelling of the excitation at the appropriate physical conditions.

\subsubsection{Cloud morphology and kinematics}

Figure 11 is an interpolated integrated intensity map of the $\mathrm{C}^{18} \mathrm{O} J=2-1$ profiles shown in Fig. A.1, in Appendix A. The interpolation of the data was done by using a three point planar interpolation routine in XS. The map is oriented in (RA, Dec), and offsets are given in arcseconds from $\mathrm{Sgr} \mathrm{A}^{*}$. In this figure, the well-known velocity gradient along the molecular belt region naturally separates all the different objects in this region. To reveal the kinematics of some of the objects observed in $\mathrm{C}^{18} \mathrm{O}$ emission, multiple position-velocity diagrams were produced, see Appendix B. The diagrams are drawn at constant Galactic longitudes, with spacings of $22.5^{\prime \prime}$ beginning at the NE end of Fig. 11. The positions of the velocity cuts are marked with letters in Fig. A.1. in Appendix A, corresponding to Figs. B.1, and B.2. in Appendix B.

The $+50 \mathrm{~km} \mathrm{~s}^{-1}$ cloud is seen as the large $\mathrm{C}$-shaped object in yellow shades $\mathrm{E}$ and $\mathrm{NE}$ of Sgr $\mathrm{A}^{*}$. It is resolved into a clumpy 


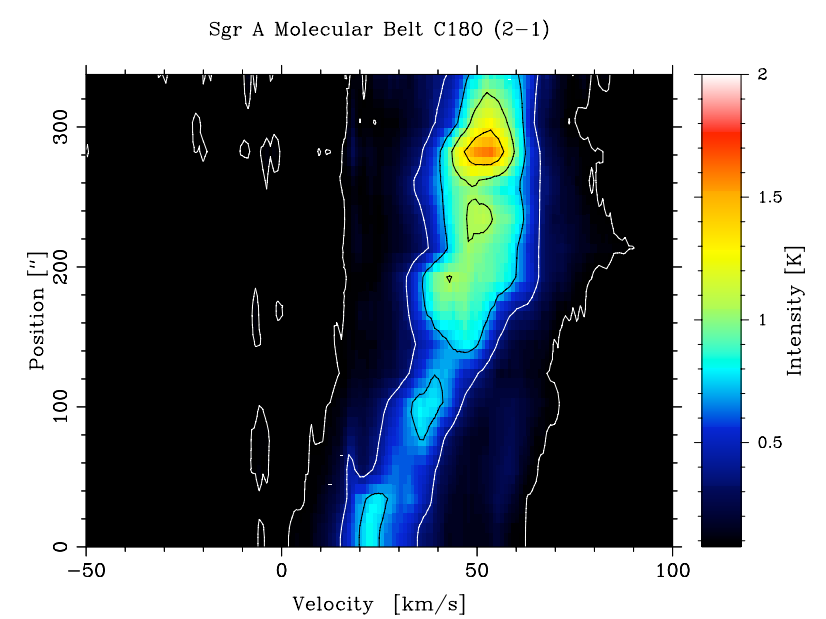

Fig. 12. Position-velocity diagram of $\mathrm{C}^{18} \mathrm{O} J=2-1$ observations along the molecular belt, the red diagonal line from A to B, in Fig. 11. The zero position is inside the $+20 \mathrm{~km} \mathrm{~s}^{-1}$ cloud at A, in Fig. 11 .

structure, with four major components marked I, II, III and IV, in Fig. 11. Component $I$ is the brightest one with a maximum integrated intensity of $45 \mathrm{~K} \mathrm{~km} \mathrm{~s}^{-1}$, while Component II has a slightly lower intensity of about $42 \mathrm{~K} \mathrm{~km} \mathrm{~s}^{-1}$. Components III and IV are seen at almost the same maximum intensity of about $36 \mathrm{~K} \mathrm{~km} \mathrm{~s}^{-1}$. Components I, II and III seem to be situated on the same plateau of enhanced emission outside of the Sgr A East shell. The projected dimensions of the Components I, II and III in Fig. 11 are roughly $75^{\prime \prime} \times 50^{\prime \prime}$ corresponding to a size of $3 \times 2 \mathrm{pc}$, and and the smaller Component IV is about $20^{\prime \prime} \times 35^{\prime \prime}$ $(0.8 \times 1.5 \mathrm{pc})$.

The W part of the $+50 \mathrm{~km} \mathrm{~s}^{-1}$ cloud is characterized by an intensity gradient, especially around (RA, Dec) offset of $\left(90^{\prime \prime}\right.$, $\left.60^{\prime \prime}\right)$. This, and the curved shape of W part of the $+50 \mathrm{~km} \mathrm{~s}^{-1}$ cloud, fits well with the outline of the Sgr A East shell contour in the figure. This supports the idea that the $+50 \mathrm{~km} \mathrm{~s}^{-1}$ cloud is located on the outside of the Sgr A East shell which is ploughing into the $+50 \mathrm{~km} \mathrm{~s}^{-1}$ cloud as discussed e.g. by Jackson et al. (1993) and Martin et al. (2012).

The velocity width of Components I and II is approximately $40 \mathrm{~km} \mathrm{~s}^{-1}$, between 30 and $70 \mathrm{~km} \mathrm{~s}^{-1}$ at the $\sim 2 \sigma$ level, see Fig. B.1a-c in Appendix B. The protrusion of Components I and II into the break in the Sgr A East shell, observed by Tsuboi et al. (2009), is also traced in our $\mathrm{C}^{18} \mathrm{O}$ observations shown in Fig. 11.

Southeast of Component I and E of Component III, the emission falls off with a steep gradient against a convex structure. Component IV appears as a separate clump projected on the $\mathrm{N}$ part of the $+50 \mathrm{~km} \mathrm{~s}^{-1}$ cloud, observed at opposite velocities of about -50 to $-80 \mathrm{~km} \mathrm{~s}^{-1}$, see Figs. B.1b-e in Appendix B.

In Fig. B.1f in Appendix B, we note a hook-like structure in the position-velocity diagram along the $\mathrm{C}-\mathrm{D}$ line in Fig. 11, indicating a line-of-sight decline of velocity from about $50 \mathrm{~km} \mathrm{~s}^{-1}$ in the molecular belt to about $20 \mathrm{~km} \mathrm{~s}^{-1}$ at the latitude offset of Sgr A*, which is at $14^{\prime \prime}$. This hook-like structure also appears in Figs. B.1d-h, and B.2i in Appendix B, at velocities between -45 and $50 \mathrm{~km} \mathrm{~s}^{-1}$. A rough estimate of the extent of this feature is between an (RA) offset of 95" and -20", and (Dec) offset between $-40^{\prime \prime}$, and $100^{\prime \prime}$, which resembles most of the blue-shifted high-velocity cloud in Genzel et al. (1990). The latitude extent of the hook-like feature seems to decline with longitude offsets from Sgr A*.

The three components $\mathrm{A}, \mathrm{B}$, and $\mathrm{C}$ of the four compact $\mathrm{H}_{\mathrm{II}}$ regions, detected by Ekers et al. (1983), on the
E side of the $+50 \mathrm{~km} \mathrm{~s}^{-1}$ cloud, are seen projected upon our Component I. The fourth compact $\mathrm{H}_{\text {II }}$ region, D, partly located in the $+50 \mathrm{~km} \mathrm{~s}^{-1}$ cloud (Mills et al. 2011), is seen as a feature between the Components I and III in Fig. 11, at an (RA, Dec) offset position of $\left(150^{\prime \prime}, 10^{\prime \prime}\right)$.

The interaction between molecular clouds and shocked gas from expanding supernova remnant shells can be probed by $1720 \mathrm{MHz}$ OH masers (e.g. Elitzur 1978; Lockett et al. 1999; Yusef-Zadeh et al. 1996). In Fig. 11 we have superimposed nineteen $1720 \mathrm{MHz} \mathrm{OH}$ masers, between velocities of 30 and $70 \mathrm{~km} \mathrm{~s}^{-1}$, observed by (Yusef-Zadeh et al. 1996, 2001; Karlsson et al. 2003; and Sjouwerman \& Pihlström 2008). Those masers are seen at projected intersections of the Sgr A East shell, Components I, II and III, and furthermore between the SNR (G359.92-0.09) and the molecular belt. This seems to confirm interactions between the Sgr A East shell and the $+50 \mathrm{~km} \mathrm{~s}^{-1}$ cloud, the molecular belt, and Components I, II and III, respectively. Furthermore, there is indication of interaction between the SNR (G359.92-0.09), the Sgr A East shell and the molecular belt, by the maser sources surrounding the SNR (G359.92-0.09) in Fig. 11.

Figure 12 shows the position-velocity cut along the molecular belt, the A-B line in Fig. 11. The velocity decreases with about $0.5 \mathrm{~km} \mathrm{~s}^{-1} \mathrm{pc}^{-1}$, in the $\mathrm{N}$ part of the molecular belt, approximately within the extent of the $+50 \mathrm{~km} \mathrm{~s}^{-1}$ cloud. Outside of the $+50 \mathrm{~km} \mathrm{~s}^{-1}$ cloud the velocity decreases from about $50 \mathrm{~km} \mathrm{~s}^{-1}$ to about $23 \mathrm{~km} \mathrm{~s}^{-1}$ where the molecular belt intersects with the $+20 \mathrm{~km} \mathrm{~s}^{-1}$ cloud, with an average gradient of $3.9 \mathrm{~km} \mathrm{~s}^{-1} \mathrm{pc}^{-1}$. The bright clump at about $285^{\prime \prime}$ on the $y$-axis, in Fig. 12, and at a velocity of about $52 \mathrm{~km} \mathrm{~s}^{-1}$, corresponds to Component I in Fig. 11.

The $\mathrm{N}$ part of the $+20 \mathrm{~km} \mathrm{~s}^{-1}$ cloud is seen $\mathrm{S}$ of Sgr A* in cyan and green colours in Fig. 11, where the outline of the $+20 \mathrm{~km} \mathrm{~s}^{-1}$ cloud is seen pointing in a NW direction in the figure. We note an interesting characteristic at approximately (RA, Dec) offsets of $\left(-80^{\prime \prime},-80^{\prime \prime}\right)$, namely a 90 -degree bend to the $\mathrm{NE}$ pointing to the SW inner part of the CND.

Figure B.2n is a position-velocity diagram through the $+20 \mathrm{~km} \mathrm{~s}^{-1}$ cloud, along the E-F line in Fig. 11 . We especially note two velocity components in the figure - the $+20 \mathrm{~km} \mathrm{~s}^{-1}$ cloud between $\sim 10 \mathrm{~km} \mathrm{~s}^{-1}$ and $40 \mathrm{~km} \mathrm{~s}^{-1}$, and an isolated narrow feature centred at $55 \mathrm{~km} \mathrm{~s}^{-1}$. A significant character of the radial velocity vector of the $+20 \mathrm{~km} \mathrm{~s}^{-1}$ cloud in Figs. B.1e, f, and B. $2 \mathrm{j}-\mathrm{O}$ in Appendix B, is its $\mathrm{S}$-shape, i.e. the radial velocity has two inflection points. In Fig. 11, the inflection points are located at (RA, Dec) offsets $\left(0^{\prime \prime},-160^{\prime \prime}\right)$, approximately $6 \mathrm{pc} \mathrm{S}$ of Sgr A*, and at (RA, Dec) offsets of $\left(-70^{\prime \prime},-120^{\prime \prime}\right)$, about $6 \mathrm{pc}$ SW of Sgr A*. At the position of the latter, a clump of slightly enhanced intensity is seen in Fig. 11.

The Odin C I profile in Fig. 9c, of the $+20 \mathrm{~km} \mathrm{~s}^{-1}$ cloud, reveals two velocity components of almost equal intensity. Component 1 is seen at a velocity of $22 \mathrm{~km} \mathrm{~s}^{-1}$, and Component 2 at a velocity of $57 \mathrm{~km} \mathrm{~s}^{-1}$. The profiles in Fig. 9c, of CO $J=7-6$, CO $J=5-4$, and CO $J=4-3$, at the position of the $+20 \mathrm{~km} \mathrm{~s}^{-1}$ cloud, also reveal weak signs of a secondary velocity component in the redward wing of the spectra, at approximately $55 \mathrm{~km} \mathrm{~s}^{-1}$. Furthermore, a narrow faint emission component in $\mathrm{C}^{18} \mathrm{O}$ can be seen in Figs. B.2k-o in Appendix B at a velocity of $55 \mathrm{~km} \mathrm{~s}^{-1}$, and a width of less than $10 \mathrm{~km} \mathrm{~s}^{-1}$. A decreasing offset latitude of the faint emission component is noted in Figs. B.2k-o in Appendix B, which corresponds to a SW direction in Fig. 11. 


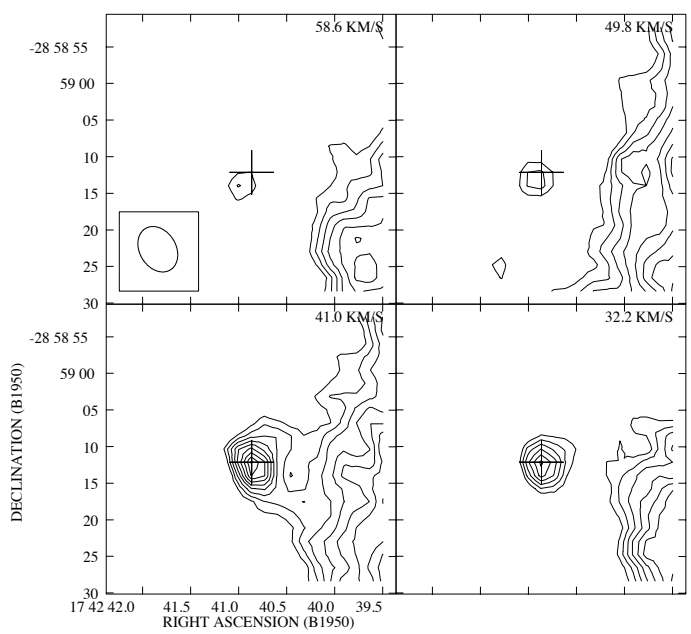

Fig. 13. $\mathrm{OH}$ absorption against the compact $\mathrm{H}$ II region $\mathrm{D}$ at $1667 \mathrm{MHz}$ observed with the VLA. The position of the compact $\mathrm{H}_{\text {II }}$ region D is marked with a plus sign. The lowest contour level is $45 \mathrm{mJy} / \mathrm{beam}$ $(\sim 2.5 \sigma)$ and the contour spacing is $5 \mathrm{mJy} / \mathrm{beam}$. The size of the beam, $7^{\prime \prime} \times 5^{\prime \prime}$, is shown at the lower left corner in the uppermost left figure.

\subsubsection{The compact $\mathrm{H} \|$ region $\mathrm{D}$}

The group of four compact $\mathrm{H}_{\text {II }}$ regions at $\sim 165^{\prime \prime}$ in projection NE of Sgr A* was first detected by Ekers et al. (1983). All four regions, identified from $\mathrm{N}$ to $\mathrm{S}$ as $\mathrm{A}-\mathrm{D}$, are projected near the rim of the Sgr A East continuum radio source, in the $\mathrm{E}$ part of the $+50 \mathrm{~km} \mathrm{~s}^{-1}$ cloud.

The compact $\mathrm{H}$ II regions $\mathrm{A}-\mathrm{C}$ are not seen in absorption towards the $+50 \mathrm{~km} \mathrm{~s}^{-1}$ cloud, which was interpreted as being foreground objects by Karlsson et al. (2003). However, strong $\mathrm{OH}$ absorption against the compact $\mathrm{H}$ II region $\mathrm{D}$ at $1667 \mathrm{MHz}$ is observed at 32 and $41 \mathrm{~km} \mathrm{~s}^{-1}$, and weak absorption at 50 and $59 \mathrm{~km} \mathrm{~s}^{-1}$, see Fig. 13. At $1665 \mathrm{MHz}$ strong OH absorption is observed at $41 \mathrm{~km} \mathrm{~s}^{-1}$, and weak absorption at 32, and $50 \mathrm{~km} \mathrm{~s}^{-1}$. There is no $\mathrm{OH}$-absorption in the adjacent velocity channels (24 and $68 \mathrm{~km} \mathrm{~s}^{-1}$, respectively), either at 1667 or at $1665 \mathrm{MHz}$. By Gaussian fitting to the $1667 \mathrm{MHz}$ profile, the maximum absorption was found at $43 \mathrm{~km} \mathrm{~s}^{-1}$, and the $\Delta V_{\text {FWHM }}=22 \mathrm{~km} \mathrm{~s}^{-1}$. The $\mathrm{OH}_{1667}$ optical depth and column density in front of the compact $\mathrm{H}_{\text {II }}$ region $\mathrm{D}$ were calculated as described in Sect. 3.1.1, assuming $T_{\mathrm{ex}}=40 \mathrm{~K}$, see Table 9.

For a further investigation of the compact $\mathrm{H}_{\text {II }}$ region $\mathrm{D}$, we used our $\mathrm{C}^{18} \mathrm{O}$ data and produced a position-velocity cut in the $\mathrm{C}^{18} \mathrm{O} J=2-1$ transition through its position. However, the emission is blended with the emission of the $+50 \mathrm{~km} \mathrm{~s}^{-1}$ cloud, and to reduce this effect we averaged two position-velocity cuts on each side of the compact $\mathrm{H}_{\text {II }}$ region $\mathrm{D}$ cut, and subtracted this from the position-velocity cut through the compact $\mathrm{H}$ in region D. The resulting position-velocity diagram is shown in Fig. 14, where the source is seen in $\mathrm{C}^{18} \mathrm{O} J=2-1$ between velocities of 35 and $62 \mathrm{~km} \mathrm{~s}^{-1}$. There seem to be two axes of emission, one at a position of $35^{\prime \prime}$ on the $y$-axis, and the other at $55^{\prime \prime}$, and the size of the source is approximately $30^{\prime \prime}(1.2 \mathrm{pc})$ in the latitude direction. The velocity increases with about $14 \mathrm{~km} \mathrm{~s}^{-1} \mathrm{pc}^{-1}$ in the NW direction. The large empty area at latitude positions above the $\mathrm{H}$ II region $\mathrm{D}$ in Fig. 14, shows that interference from the $+50 \mathrm{~km} \mathrm{~s}^{-1}$ cloud and the molecular belt was successfully eliminated by the subtraction of the averaged cuts from the cut through the compact $\mathrm{H}_{\text {II }}$ region D.
C180 (2-1) Region D

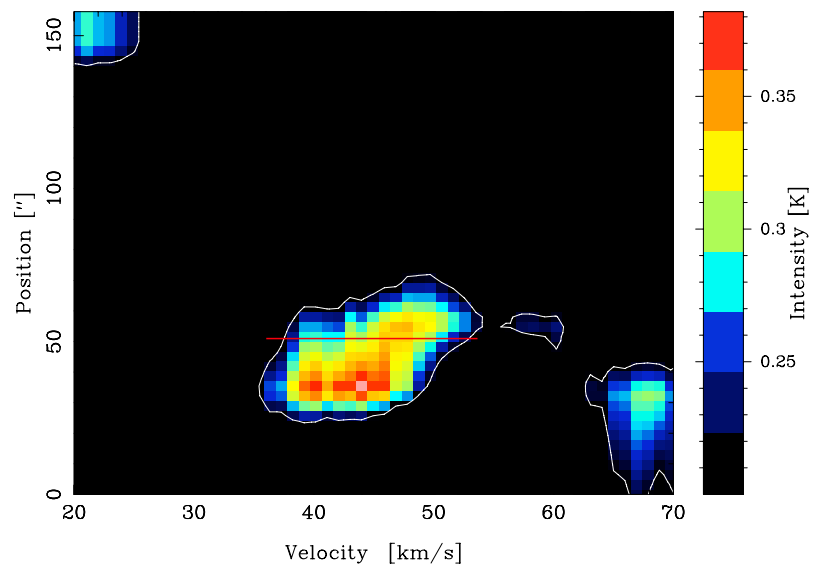

Fig. 14. Position-velocity cut of $\mathrm{C}^{18} \mathrm{O} J=2-1$ through the position of the compact $\mathrm{H}$ in region $\mathrm{D}$, the line $\mathrm{G}-\mathrm{H}$ in Fig. 11, see Sect. 3.5.3. The red line marks the position of the compact $\mathrm{H}_{\text {II }}$ region $\mathrm{D}$ with reference to the point $\mathrm{G}$ in Fig. 11.

Table 9. $\mathrm{OH}$ absorption against the compact $\mathrm{H}$ in region $\mathrm{D}$ at $1667 \mathrm{MHz}$.

\begin{tabular}{lc}
\hline \hline (B1950) & $17^{\mathrm{h}} 42^{\mathrm{m}} 40^{\mathrm{s}} 87,-28^{\circ} 59^{\prime} 12^{\prime \prime} 1$ \\
Maximum absorption & $82 \mathrm{mJy} / \mathrm{beam}$ \\
$\Delta V_{\mathrm{FWHM}}$ & $22 \mathrm{~km} \mathrm{~s}^{-1}$ \\
$\tau_{1667}$ & $4.8(\mathrm{NA} /-1.7)$ \\
$N(\mathrm{OH})$ & $\leq 0.9 \times 10^{18} \mathrm{~cm}^{-2}$ \\
\hline
\end{tabular}

\subsection{Molecular column density ratios and abundances}

Table 10 is a summary of the column density ratios of the observed species, together with abundance estimates calculated as $X($ species $)=N($ species $) / N\left(\mathrm{H}_{2}\right)$. Note that we use a constant ratio of 250 for $\mathrm{H}_{2} \mathrm{O} / \mathrm{H}_{2}^{18} \mathrm{O}$ (Wilson \& Rood 1994), for the $+50 \mathrm{~km} \mathrm{~s}^{-1}$ cloud, the CND, the $+20 \mathrm{~km} \mathrm{~s}^{-1}$ cloud, the EMR, and the $3-\mathrm{kpc}$ arm. The values of $N\left(\mathrm{H}_{2}\right)$ for the $+50 \mathrm{~km} \mathrm{~s}^{-1}$ cloud, the CND, and the $+20 \mathrm{~km} \mathrm{~s}^{-1}$ cloud are taken from our $\mathrm{C}^{18} \mathrm{O}$ results in Table 8. For the spiral arm features we have used the averaged values for the three observed main positions in Table 3 by Sandqvist et al. (2003). The abundance results will be discussed in Sect. 4.8 .

\section{Discussion}

The molecular clouds at the GC are clumpy, dense, warm and often turbulent. Shocks associated with supernovae and cloudcloud collisions are also common among GC molecular clouds and may trigger star formation, often manifested by the detection of $1720 \mathrm{MHz}$ maser sources excited by SNR shocks (e.g. Yusef-Zadeh et al. 2001; Sjouwerman \& Pihlström 2008) and recent star formation is seen in terms of compact $\mathrm{H}$ II regions, e.g. Ekers et al. (1983). Expulsion of clumps and collisions between clumps may develop into streamers and molecular clouds of different morphology and size, that ultimately may lose angular momentum and travel towards the dynamical centre of the Galaxy.

\subsection{The $+50 \mathrm{~km} \mathrm{~s}^{-1}$ cloud}

The observed velocity line width of $\mathrm{OH}$ in the $+50 \mathrm{~km} \mathrm{~s}^{-1}$ cloud is about $50 \mathrm{~km} \mathrm{~s}^{-1}$ considering Pos. 1, 2, and 3. For GMCs in 
R. Karlsson et al.: $\mathrm{OH}, \mathrm{H}_{2} \mathrm{O}, \mathrm{CO}, \mathrm{NH}_{3}$, and $\mathrm{C} \mathrm{I}$ in the Sgr A complex

Table 10. Column density ratios and estimated abundances.

\begin{tabular}{lccccccccc}
\hline \hline Source & $\mathrm{OH} / \mathrm{H}_{2}^{18} \mathrm{O}$ & $\mathrm{OH} / \mathrm{H}_{2} \mathrm{O}$ & $\mathrm{OH} / \mathrm{NH}_{3}$ & $\mathrm{OH} / \mathrm{C}_{\mathrm{I}}$ & $\mathrm{H}_{2} \mathrm{O} / \mathrm{NH}_{3}$ & $X(\mathrm{OH})$ & $X\left(\mathrm{H}_{2} \mathrm{O}\right)$ & $X\left(\mathrm{H}_{2}^{18} \mathrm{O}\right)$ & $X\left(\mathrm{NH}_{3}\right)$ \\
\hline$+50 \mathrm{~km} \mathrm{~s}^{-1} \mathrm{Cl}$. & $4.4 \times 10^{4}$ & $1.8 \times 10^{2}$ & - & $\leq 0.4$ & & $7.5 \times 10^{-6}$ & $4.2 \times 10^{-8}$ & $1.7 \times 10^{-10}$ & \\
CND & $1.3 \times 10^{5}$ & $5.3 \times 10^{2}$ & - & $\leq 1.3$ & & $3.6 \times 10^{-5}$ & $6.8 \times 10^{-8}$ & $2.8 \times 10^{-10}$ & \\
$+20 \mathrm{~km} \mathrm{~s}^{-1} \mathrm{Cl}$. & $2.0 \times 10^{5}$ & $7.5 \times 10^{2}$ & - & $\leq 1$ & & $1.0 \times 10^{-5}$ & $1.6 \times 10^{-8}$ & $0.6 \times 10^{-10}$ & \\
EMR near side & $1.0 \times 10^{4}$ & 70 & - & - & & & & & \\
Local/Sgr arm & - & - & $6.7 \times 10^{2}$ & - & & $1.8 \times 10^{-6}$ & & & $5.7 \times 10^{-9}$ \\
$-30 \mathrm{~km} \mathrm{~s}$ & -1 & - & $1.8 \times 10^{2}$ & $1.1 \times 10^{3}$ & - & 5.9 & $5.8 \times 10^{-6}$ & $3.1 \times 10^{-8}$ & $10^{-9}$ \\
3-kpc arm & - & - & $1.2 \times 10^{3}$ & - & & $3.5 \times 10^{-6}$ & & & $3.0 \times 10^{-9}$ \\
\hline
\end{tabular}

Notes. Based upon column densities given in Tables 4-8. Abundances are calculated as $X($ species $)=N($ species $) / N\left(\mathrm{H}_{2}\right)$.

the GC, Miyazaki \& Tsuboi (1999), have shown a statistical relation between the cloud radius and the velocity line width. For a cloud with a radius of $5 \mathrm{pc}$, approximately the size of the +50 and $+20 \mathrm{~km} \mathrm{~s}^{-1}$ clouds, the line width would be about $25 \mathrm{~km} \mathrm{~s}^{-1}$. The observed excess line width of these clouds is of the same order and is considered to be a result of collision with the Sgr A East shell, projection effects, effect of shock waves, collision between cloud clumps, turbulence and star formation with associated bipolar outflows in the clouds.

The $\mathrm{H}_{2} \mathrm{O}$ emission is generally optically thick in the GC clouds, resulting in strong self-absorption by lower-excitation gas in the line-of-sight of the cloud. The $\mathrm{H}_{2} \mathrm{O}$ peak emission velocities are displaced by up to $20 \mathrm{~km} \mathrm{~s}^{-1}$ towards higher velocities compared to the $\mathrm{OH}$ absorption as a result of the strong self-absorption in the $\mathrm{H}_{2} \mathrm{O}$ line. The $\mathrm{H}_{2} \mathrm{O}$ self-absorption can also be revealed by the non-Gaussian shape of the blue wing of the $\mathrm{H}_{2} \mathrm{O}$ emission profiles, at Pos. 1, 2, and 3. On the other hand, the velocity difference between the $\mathrm{OH}$ absorption and the optically thin $\mathrm{H}_{2}^{18} \mathrm{O}$ absorption is small.

In Fig. 9 the velocity profiles of $\mathrm{C}$, the three $\mathrm{CO}$ transitions and $\mathrm{H}_{2} \mathrm{O}$ are shown. The velocity peaks of the $+50 \mathrm{~km} \mathrm{~s}^{-1}$ cloud are displaced by velocities higher than the nominal $50 \mathrm{~km} \mathrm{~s}^{-1}$ in all five transition profiles. Even for the CO transitions, this is most likely an effect of self-absorption in the optically thick environment of the cloud. We note that the CO lines appear to have two velocity components, at the $+50 \mathrm{~km} \mathrm{~s}^{-1}$ cloud and the CND, while the $C_{I}$ line has only a single velocity component. The higher velocity component of the CO lines seems to have the same velocity as the $C_{\text {I }}$ peak. The smeared out blue wing of the CO $J=5-4$ and $J=7-6$ profiles in the $+50 \mathrm{~km} \mathrm{~s}^{-1}$ cloud and the CND is indicative of shock activity in those objects.

Inside the $+50 \mathrm{~km} \mathrm{~s}^{-1}$ cloud, the dominating components, labelled I-IV in Fig. 11, have different characteristics. Component II has a steep intensity gradient towards the Sgr A East shell, and also seems to penetrate into the opening of the shell, NE of $\mathrm{Sgr} \mathrm{A}^{*}$, at the contact point between the $+50 \mathrm{~km} \mathrm{~s}^{-1}$ cloud and the Sgr A East shell (cf. Tsuboi et al. 2009). The steep intensity gradient in the SW part of Component II, seen in Fig. 11, indicates a compression of the cloud by the expanding Sgr A East shell. The points of contact between the shell and adjacent clouds are also manifested by the observations of the shock-excited $1720 \mathrm{MHz} \mathrm{OH}$ masers in this area (Yusef-Zadeh et al. 1996, 2001; Karlsson et al. 2003; Sjouwerman \& Pihlström 2008), which are overlaid in Fig. 11.

Component $\mathrm{I}$ is the brightest one and has a triangular shape with steep intensity gradients on all its sides. It is seen projected on the positions of the three compact $\mathrm{H}_{\text {II }}$ regions $\mathrm{A}, \mathrm{B}$ and $\mathrm{C}$. Along the W edge of Component I, Sjouwerman \& Pihlström (2008) observed $1720 \mathrm{MHz} \mathrm{OH}$ masers along a line from NW to SE. The curvature of the line connecting these masers seems to fit well with the curvature of Component I on the side facing to the masers. It therefore seems likely that the $\mathrm{OH}$ masers are located on the rim of Component I, and are being triggered by a shock wave that forms the intensity gradient on the $\mathrm{W}$ edge of Component I. The Component I could itself be a result of interaction with the compact $\mathrm{H}_{\text {II }}$ regions $\mathrm{A}, \mathrm{B}$, and $\mathrm{C}$.

Components I and III show steep intensity gradients on the E side of the $+50 \mathrm{~km} \mathrm{~s}^{-1}$ cloud, and seem to be compressed by the "expanding shell" located in the SE part of the $+50 \mathrm{~km} \mathrm{~s}^{-1}$ cloud. The expanding shell is suggested to be a $7 \times 10^{4}$ years old supernova remnant by Tsuboi et al. (2011). This internal expanding shell is also seen as a hole in the $\mathrm{C}_{\mathrm{I}}$ emission observed by Poglitsch et al. (1991). In Fig. 11 we observe a compression at (RA, Dec) offset of $\left(170^{\prime \prime},-25^{\prime \prime}\right)$, that likely is the NW rim of the supernova remnant expanding into the $+50 \mathrm{~km} \mathrm{~s}^{-1}$ cloud observed by Tsuboi et al. (2011).

Our investigations have strengthened the view that the $+50 \mathrm{~km} \mathrm{~s}^{-1}$ cloud is dominated by shock activities from the expanding Sgr A East shell, star formation and possibly by a supernova remnant and the blue-shifted high-velocity cloud. The $\mathrm{OH}$ abundance in the $+50 \mathrm{~km} \mathrm{~s}^{-1}$ cloud is lower than in the $+20 \mathrm{~km} \mathrm{~s}^{-1}$ cloud, $1: 1.5$. The $\mathrm{H}_{2} \mathrm{O}$ abundance is, however, higher in the $+50 \mathrm{~km} \mathrm{~s}^{-1}$ cloud than in the $+20 \mathrm{~km} \mathrm{~s}^{-1}$ cloud, $2: 1$, see Table 10 . This may indicate that $\mathrm{OH}$ has been subject to more shock conversion leading to $\mathrm{H}_{2} \mathrm{O}$ enrichment in the $+50 \mathrm{~km} \mathrm{~s}^{-1}$ cloud than is the case in the $+20 \mathrm{~km} \mathrm{~s}^{-1}$ cloud.

\subsubsection{The compact HII region D}

Several studies have been made of the four compact $\mathrm{H}_{\text {II }}$ regions, e.g. by Ekers et al. (1983), Goss et al. (1985), and recently by Yusef-Zadeh et al. (2010) and Mills et al. (2011). This group of compact $\mathrm{H}$ II regions is likely to be the closest site of on-going star formation in the Galactic centre, and the compact $\mathrm{H}_{\text {II }}$ region $\mathrm{D}$ is the youngest one, a few times $10^{4}$ years, and also the most compact region (Yusef-Zadeh et al. 2010). The radial velocities of the four regions were observed at 43 to $52 \mathrm{~km} \mathrm{~s}^{-1}$ (Goss et al. 1985). The spectral energy distribution of the compact H II region $\mathrm{D}$ indicates that the central star is a $25 \pm 3 M_{\odot}$ O9-B0 star (Yusef-Zadeh et al. 2010).

The compact $\mathrm{H}$ II region $\mathrm{D}$ is located between the $\mathrm{SW}$ and NE sides of Components I and III, respectively, see Fig. 11. Tsuboi et al. (2009) suggest that the four compact $\mathrm{H}$ II regions are placed on the outside sunward side of an expanding molecular shell inside the $+50 \mathrm{~km} \mathrm{~s}^{-1}$ cloud, see Fig. 8 in their paper. Mills et al. (2011) argue that the compact $\mathrm{H}_{\mathrm{II}}$ region $\mathrm{D}$ is located in, or partly inside the $+50 \mathrm{~km} \mathrm{~s}^{-1}$ cloud, partly based on results by Karlsson et al. (2003). Our concatenated high-resolution VLA data set of $\mathrm{OH}$ absorption against the $+50 \mathrm{~km} \mathrm{~s}^{-1}$ cloud, supports the view of Mills et al. (2011). As seen in Fig. 13, there are 
clear indications of $\mathrm{OH}$ gas in front of the compact $\mathrm{H}_{\text {II }}$ region $\mathrm{D}$, at velocities between 32 and $59 \mathrm{~km} \mathrm{~s}^{-1}$.

Serabyn et al. (1992) note that the four compact H II regions are situtated outside of the expanding Sgr A East shell, and not reached by the blast wave front from Sgr A East. In this study we find the source D seemingly being "squeezed" between the Components I and III, seen in Fig. 11. We suggest that there is a relation between the forming of the compact $\mathrm{H}$ II region $\mathrm{D}$, and the Components I and III. We also note that since the compact $\mathrm{H}$ II region $\mathrm{D}$ is located inside, or at least partly inside, of the $+50 \mathrm{~km} \mathrm{~s}^{-1}$ cloud, the physical and chemical characteristics of this region should be different from the three compact $\mathrm{H}$ II regions $\mathrm{A}, \mathrm{B}$ and $\mathrm{C}$.

It is interesting to note that Mills et al. (2011) also find two emission components, although on a much smaller scale than our two components, and in an E-W direction, which they interpret as the existence of a disk-like structure. The direction of the two components detected in this study, may be indicative of a large scale outflow from the compact $\mathrm{H}_{\mathrm{II}}$ region $\mathrm{D}$.

\subsection{The molecular belt}

The hook-like structure that we observe in $\mathrm{C}^{18} \mathrm{O}$ emission, described in Sect. 3.5.2, agrees well with the geometry of the molecular belt as outlined by the 1.3-mm dust emission by Zylka et al. (1990) and 2-mm $\mathrm{H}_{2} \mathrm{CO}$ emission observed by Sandqvist (1989), where the projected width of the molecular belt is shown to decline from NE to $\mathrm{S}$, with a marked contraction at the position of Sgr $\mathrm{A}^{*}$. The same morphology of the molecular belt is also seen in our $\mathrm{C}^{18} \mathrm{O}$ data in Figs. 11, and B.1. in Appendix B.

The dip of integrated intensity around (RA, Dec) offset of $\left(60^{\prime \prime},-90^{\prime \prime}\right)$ in Fig. 11 seems to indicate a lack of gas in this part of the molecular belt. In a paper by Coil \& Ho (2000), a supernova remnant is discussed, the SNR (G359.92-0.09), which interacts with Sgr A East, the molecular belt and the $+20 \mathrm{~km} \mathrm{~s}^{-1}$ cloud. This feature can also be seen in Yusef Zadeh \& Morris (1987), as a very faint arc-like structure around (RA, Dec) of $\left(17^{\mathrm{h}} 42^{\mathrm{m}} 36^{\mathrm{s}},-29^{\circ} 00^{\prime} 30^{\prime \prime}\right)$, with its convex side facing to $\mathrm{N}$. Along the NW part of the arc, Yusef-Zadeh et al. (2001) observed shock-excited $1720 \mathrm{MHz} O \mathrm{OH}$ masers. Furthermore, in the model by Herrnstein \& Ho (2005), the SNR (G359.92-0.09) is depicted as a circular feature partly lying behind the molecular belt and the $+20 \mathrm{~km} \mathrm{~s}^{-1}$ cloud.

In our $\mathrm{C}^{18} \mathrm{O}$ map, shown in Fig. 11, we have fitted an arc to the observed depletion of $\mathrm{C}^{18} \mathrm{O}$ gas in the molecular belt, the masers at the south rim of Sgr A East, and the single maser at (RA, Dec) offset of $\left(90^{\prime \prime},-155^{\prime \prime}\right)$. We find that the position of the N rim of the SNR (G359.92-0.09), seen in the paper by Yusef-Zadeh \& Morris (1987), agrees well with the arc we fitted in Fig. 11, and the model presented by Herrnstein \& Ho (2005). It is therefore likely that the SNR (G359.92-0.09) is related to the depletion of $\mathrm{C}^{18} \mathrm{O}$ gas in the molecular belt. The SE masers are located in projection at an assumed collision front between the Sgr A East shell and the SNR (G359.92-0.09), and these masers are possibly triggered by the collision of these two supernova remnant shells.

\subsection{The circumnuclear disk}

The physical conditions in the CND have been observed to vary a lot, from the rather sharp photo-ionized inner edge of the asymmetric clumpy massive torus-like CND to its more fuzzy, irregular outer edge. The CND is suggested to be fed from both the +20 and the $+50 \mathrm{~km} \mathrm{~s}^{-1}$ clouds (Jackson et al. 1993), and more recently by Coil \& Ho (2000) who discuss the $+20 \mathrm{~km} \mathrm{~s}^{-1}$ cloud as feeding the CND via the Southern Streamer. The idea that the central "cavity" within the CND has been more or less "evacuated" by stellar winds and photo-ionized by UV radiation from the central star cluster has been discussed by a number of authors, e.g., in the review papers referred to in our Introduction and most recently by Ferrière (2012).

We found that the CND is much richer in both $\mathrm{OH}$ and $\mathrm{H}_{2} \mathrm{O}$ than the +50 and $+20 \mathrm{~km} \mathrm{~s}^{-1}$ clouds, see Table 10 and further discussion in Sect. 4.7.

\subsection{The $+20 \mathrm{~km} \mathrm{~s}^{-1}$ cloud}

The structure of the $+20 \mathrm{~km} \mathrm{~s}^{-1}$ cloud is complex. Basically, it is suggested to be a massive molecular cloud composed of two components, the cloud itself and the Southern Streamer in the northern part. It seems possible that the $+20 \mathrm{~km} \mathrm{~s}^{-1}$ cloud is fed with gas via the molecular belt, since there are indications of physical interaction between the two objects (cf. Herrnstein \& Ho 2005). Furthermore, the $+20 \mathrm{~km} \mathrm{~s}^{-1}$ cloud is impacted by the supernova remnant SNR (G359.92-0.09), located E of and partly behind the cloud and the molecular belt as seen from the Sun (Herrnstein \& Ho 2005; and references therein).

The outline of the part of the $+20 \mathrm{~km} \mathrm{~s}^{-1}$ cloud seen in the $\mathrm{C}^{18} \mathrm{O}$ map (Fig. 11), is interesting. The NW part of the cloud that makes a sharp turn in the direction of $\mathrm{Sgr} \mathrm{A}^{*}$, and the SW inflection point, described in Sect. 3.5.2, are both located in projection at the SW part of the CND, at which position both the inner and outer parts of the CND are seen. All three observed indications, the change of velocity, the sharp bend of the $+20 \mathrm{~km} \mathrm{~s}^{-1}$ cloud, and the enhanced intensity in $\mathrm{C}^{18} \mathrm{O}$ emission, suggest a scenario of the $+20 \mathrm{~km} \mathrm{~s}^{-1}$ cloud as interacting with the $\mathrm{S}$ part of the CND.

The other inflection point in the $+20 \mathrm{~km} \mathrm{~s}^{-1}$ cloud, $\mathrm{S}$ of $\mathrm{Sgr} \mathrm{A}^{*}$, is located where the molecular belt intersects with the $+20 \mathrm{~km} \mathrm{~s}^{-1}$ cloud, at (RA, Dec) offset of $\left(0^{\prime \prime},-160^{\prime \prime}\right)$, where a slightly reduced integrated emission is seen in Fig. 11. As seen in the figure, this position is also near the location where the SNR (G359.92-0.09) seems to protrude into the $+20 \mathrm{~km} \mathrm{~s}^{-1}$ cloud.

\subsection{The wide velocity feature}

The WVF is seen at Pos. 1-6 in our OH observations. Pos. 3 is considered to sample most of the blue-shifted high-velocity cloud, E and NE of Sgr A*, as observed by Genzel et al. (1990), see Fig. 9 in their paper. This emission extends approximately between $0^{\prime \prime}$ and $110^{\prime \prime}$ RA offset from Sgr A*, and $-30^{\prime \prime}$ to $110^{\prime \prime}$ Dec offset from $\mathrm{Sgr} \mathrm{A}^{*}$, and is suggested to be associated with the large expanding shell of gas, Sgr A East. Gaussian fits to the $\mathrm{OH}$ profile at Pos. 3 reveal an absorption feature with a centre velocity of $-32 \mathrm{~km} \mathrm{~s}^{-1}$ and a $\Delta V_{\mathrm{FWHM}}$ of $107 \mathrm{~km} \mathrm{~s}^{-1}$. Indications of the WVF are also seen in our $\mathrm{C}^{18} \mathrm{O}$ data in Figs. B.1a-e in Appendix B, at velocities approximately between -30 and $20 \mathrm{~km} \mathrm{~s}^{-1}$. The extent of the WVF is thus observed from NE to $S$ of Sgr $\mathrm{A}^{*}$ in $\mathrm{OH}$ absorption, and $\mathrm{NE}$ of $\mathrm{Sgr} \mathrm{A}^{*}$ in $\mathrm{C}^{18} \mathrm{O}$ emission. We therefore suggest that the blue-shifted high-velocity cloud is a part of the WVF. The velocity range of the WVF indicates a bubble-like morphology.

\subsection{The line-of-sight spiral arm features}

A good picture in the position-velocity space of the foreground line-of-sight spiral arms towards the Sgr A complex can be seen 


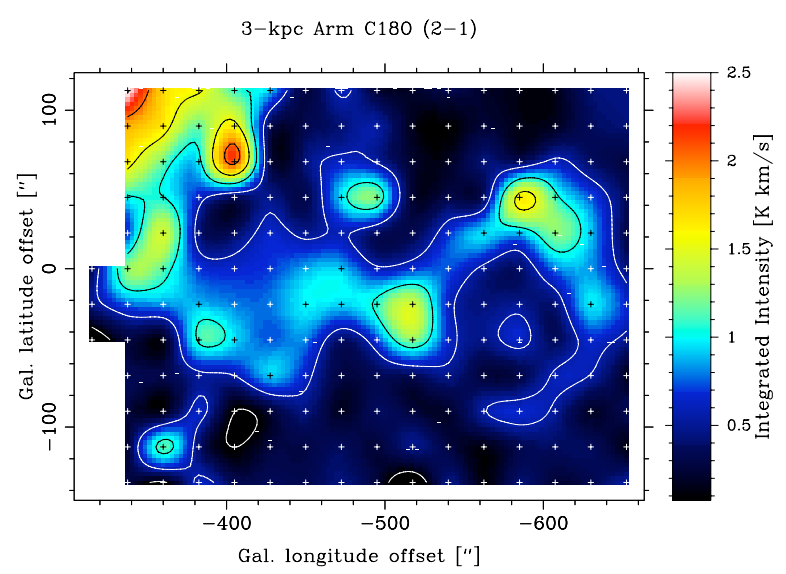

Fig. 15. $(l, b)$ diagram of the $\mathrm{C}^{18} \mathrm{O} J=2-1$ integrated emission of the 3 -kpc arm at -60 to $-45 \mathrm{~km} \mathrm{~s}^{-1}$. The lowest integrated intensity contour level is $\sim 3 \sigma$. The offsets (in arcseconds) are in the Galactic coordinate system and are relative to the position $(l, b)=\left(+5^{\prime},-3^{\prime}\right)$, and the same as in Fig. A.1. Sgr $A^{*}$ is located at $\left(-500^{\prime \prime}, 14^{\prime \prime}\right)$.

in the paper by Tanaka et al. (2011). The velocity range is about -60 to $10 \mathrm{~km} \mathrm{~s}^{-1}$.

Signatures of the narrow absorption features of the three lineof-sight spiral arm features can be traced in the $\mathrm{C}^{18} \mathrm{O}$ observations in all the position-velocity diagrams in Figs. B.1, and B.2, in Appendix B, although the features near -30 and $0 \mathrm{~km} \mathrm{~s}^{-1}$ are contaminated by other sources in the field of view, such that we were not able to separate the $-30 \mathrm{~km} \mathrm{~s}^{-1}$ arm feature in the position-velocity diagrams in Figs. B.1, and B.2, in Appendix B.

However, the $\mathrm{C}^{18} \mathrm{O}$ emission between -60 and $-45 \mathrm{~km} \mathrm{~s}^{-1}$, arising in the $3-\mathrm{kpc}$ arm, is clearly detected in all positionvelocity diagrams in Appendix B. We therefore produced an integrated intensity map of $\mathrm{C}^{18} \mathrm{O}$ at this velocity interval which is shown in Fig. 15. The wavy structure seen in the figure delineates the 3-kpc arm in $\mathrm{C}^{18} \mathrm{O}$ emission across the observed field. The bright clumps around $(l, b)$ offset of $\left(-405^{\prime \prime}, 70^{\prime \prime}\right)$, and in the upper left corner of the figure are the features of unknown origin at the location of (RA, Dec) offset of $\left(-10^{\prime \prime}, 105^{\prime \prime}\right)$ in Fig. 11 and Component IV. The structure of $\mathrm{C}^{18} \mathrm{O}$ in the $3-\mathrm{kpc}$ arm is clumpy, with a typical clump size of the order of $30^{\prime \prime}$, or $0.8 \mathrm{pc}$ at an assumed distance of the 3-kpc arm of $5 \mathrm{kpc}$ from the Sun. In Fig. 15, we also note an $80^{\prime \prime}$ (2 pc) horse-shoe shaped emission structure centred at a Galactic offset of $\left(-585^{\prime \prime},-40^{\prime \prime}\right)$, the origin of which is still unknown.

\subsection{Molecular abundances in different cloud types}

To set the scene for a discussion of the rather accurate abundances of $\mathrm{OH}, \mathrm{o}-\mathrm{H}_{2} \mathrm{O}$ and $\mathrm{o}-\mathrm{NH}_{3}$, determined from absorption lines caused by cloud regions situated in front of the warm molecular cloud cores of Sgr A, in the context of statistical observational astrochemistry, we have in Table 11 collected our own results, together with similarly accurate abundances estimated for some other absorption line targets, viz. Sgr B2 (ISO and Odin results), G10.6-0.4 (W31C; Herschel HIFI results) and W49N (Arecibo, SWAS and Herschel HIFI results). The relevant references can be found in the Table 11 footnotes. In addition we have entered some useful and interesting, and in some cases very contrasting, abundance results - albeit they may be somewhat more uncertain since they are determined from emission line analysis and hence more critically depend upon knowledge of the physical conditions than does the absorption line analysis - for the various cloud components seen in the nearby (at a distance of about $420 \mathrm{pc}$ ) giant molecular cloud Orion KL (OMC-1; ISO and Odin results) and the very nearby (at 120-160 pc) low mass molecular cloud cores rho Oph A and IRAS 16293-2422 (SWAS and Odin results). Hence most results quoted, except for the recent Herschel HIFI data, are based upon observations performed with relatively large beam sizes $\left(\approx 2^{\prime}\right.$ in case of Odin).

In view of the expected flood of overwhelming Herschel results in the near future, we will try to keep our current observation-based abundance discussion very short and concise, and with only a few references to chemistry models:

i) Water vapour. According to Table 11, the rather accurately determined $\mathrm{H}_{2} \mathrm{O}$ abundances in the nearby low-mass cloud cores rho Oph A and IRAS 16293-2422 are very low, $\left(5 \times 10^{-9}\right.$ and $2 \times 10^{-9}$, respectively, resulting from Monte-Carlo and Accelerated Lambda Iteration modelling of the self-absorbed $557 \mathrm{GHz}$ water emission lines observed by SWAS and Odin; see Ashby et al. 2000 and Hjalmarson et al. 2003). Such low abundances appear to be typical for the many quiescent molecular clouds observed by SWAS and Odin (Snell et al. 2000; Wilson et al. 2003), and presumably are results of gas-phase ionmolecule reactions (cf. Bergin et al. 2000).

The enhanced abundance of $\mathrm{H}_{2} \mathrm{O}$ of $(2-7) \times 10^{-8}$ relative to $\mathrm{H}_{2}$ observed by us in the absorbing front side of the Sgr A molecular clouds (highest in the CND but also high in the +50 and $+20 \mathrm{~km} \mathrm{~s}^{-1}$ clouds) may not be surprising in view of the observed shocks and increased UV light from newly formed stars. Sputtering of grain mantles into gas-phase by the action of shocks in fact already has been proposed as an explanation of the enhanced abundances of $\mathrm{SiO}$ and HNCO observed in the Sgr A clouds (Amo-Baladrón et al. 2011). The strong UV radiation from the central star cluster (in the CND "cavity") may further increase the desorption of water ice in the CND. The enhanced $\mathrm{OH}$ abundances also observed by us in the Sgr A clouds may in this scenario be the results of shock and photo dissociation of $\mathrm{H}_{2} \mathrm{O}$, then decreasing the high gas-phase $\mathrm{H}_{2} \mathrm{O}$ abundances produced from icy grain mantles. A combination of shock action and PDR (Photon Dominated Region) chemistry (including grain surface processes) may be needed.

The high water abundance of $10^{-7}$ relative to $\mathrm{H}_{2}$ observed in the absorbing front side of the Sgr B2 cloud envelope very likely is a result of PDR chemistry caused by the increased UV flux from newly formed stars in this very massive molecular cloud. A similarly high $\mathrm{H}_{2} \mathrm{O}$ abundance has been observed in the PDR interface region between M42 $\mathrm{H}$ II region and front side of the Orion KL molecular cloud (Wirström et al. 2006). The previously concluded PDR scenario causes us to suggest that the likewise enhanced water abundances in the $-30 \mathrm{~km} \mathrm{~s}^{-1}$ arm towards Sgr A, the 3-kpc arm towards Sgr B2 and in spiral arm clouds against W49N, as determined from absorption line analysis, also must be signs of ongoing star formation activity. These elevated $\mathrm{H}_{2} \mathrm{O}$ abundances may probably be produced by the extended PDR model (including grain surface reactions) developed by Hollenbach et al. (2009).

To show the full dynamic range of the observed water abundances we have in Table 11 also entered the even higher abundances found in the Orion KL hot core (HC) and warm compact ridge $(\mathrm{CR})$ regions as well as in the low and high velocity outflows (as estimated and discussed e.g. by Persson et al. 2007 and Olofsson et al. 2003, based upon our Odin spectral line survey and mapping of the Orion KL region). While the chemical model understanding of the $\mathrm{HC}$ and $\mathrm{CR}$ is based upon hydrogenation on cold grain surfaces and subsequent release of molecules caused by heating/photo detachment by a nearby newly formed star, the 
Table 11. Molecular abundance comparisons.

\begin{tabular}{|c|c|c|c|c|}
\hline Source & $\begin{array}{c}\text { Region } \\
\text { (Absorption feature) }\end{array}$ & $\begin{array}{l}X(\mathrm{OH}) \\
\left(\times 10^{-6}\right) \\
\end{array}$ & $\begin{array}{c}X\left(\mathrm{o}-\mathrm{H}_{2} \mathrm{O}\right) \\
\left(\times 10^{-9}\right)\end{array}$ & $\begin{array}{c}X\left(\mathrm{o}-\mathrm{NH}_{3}\right) \\
\left(\times 10^{-9}\right) \\
\end{array}$ \\
\hline $\operatorname{Sgr} \mathrm{A}^{a}$ & $\begin{array}{c}+50 \mathrm{~km} \mathrm{~s}^{-1} \text { cloud } \\
+50 \mathrm{~km} \mathrm{~s}^{-1} \text { cloud red wing } \\
\text { CND } \\
\text { CND red wing } \\
+20 \mathrm{~km} \mathrm{~s}^{-1} \text { cloud } \\
+20 \mathrm{~km} \mathrm{~s}^{-1} \text { cloud red wing } \\
\text { Local } / \mathrm{Sgr} \text { arm } \\
-30 \mathrm{~km} \mathrm{~s}^{-1} \text { arm } \\
3-\mathrm{kpc}^{2} \text { arm }\end{array}$ & $\begin{array}{c}8 \\
- \\
35 \\
- \\
13 \\
- \\
2 \\
6 \\
4\end{array}$ & $\begin{array}{c}40 \\
1000^{\S} \\
70 \\
5700^{\S} \\
20 \\
800^{\S} \\
- \\
30 \\
- \\
\end{array}$ & $\begin{array}{l}- \\
- \\
- \\
- \\
- \\
- \\
3 \\
5 \\
3\end{array}$ \\
\hline Sgr B2 & $\begin{array}{c}\text { cloud envelope } \\
\text { EMR } \\
\text { 3-kpc arm }\end{array}$ & $\begin{array}{c}2-5^{b} \\
- \\
- \\
\end{array}$ & $\begin{array}{c}100^{c, d} \\
- \\
20-400^{c, f}\end{array}$ & $\begin{array}{c}\approx 7^{c, e} \\
12^{c} \\
7^{c}\end{array}$ \\
\hline G10.06-0.4 & foreground clouds & - & - & $2(0.5-7)^{g}$ \\
\hline W49N & spiral arm clouds & $0.2 \& 1^{h}$ & $80-400^{i}$ & $2(1-7)^{j}$ \\
\hline Orion KL & $\begin{array}{c}\text { PDR interface } \\
\text { PDR bar } \\
\text { Compact ridge } \\
\text { Hot core } \\
\text { Low vel. outflow } \\
\text { High vel. outflow }\end{array}$ & $\begin{array}{l}- \\
- \\
- \\
- \\
1^{n} \\
-\end{array}$ & $\begin{array}{c}\geq 110^{k, l} \\
- \\
2800^{l} \\
12000^{l} \\
2900^{l} \\
22000^{l}\end{array}$ & $\begin{array}{c}- \\
5^{m} \\
200^{l} \\
1600^{l} \\
- \\
-\end{array}$ \\
\hline rho Oph A & core & - & $5^{\circ}$ & $0.4^{p}$ \\
\hline IRAS 16293 & core & - & $2^{q}$ & - \\
\hline
\end{tabular}

Notes. ${ }^{(\S)}$ Beam-averaged, lower abundance limit, since the comparison $\mathrm{H}_{2}$ column density very likely is overestimated (see Sect. 4.7.). ${ }^{(a)}$ From this paper, Table 10; absorption features observed by VLA and Odin. ${ }^{(b)}$ ISO result; Goicoeachea (2008), Polehampton et al. (2007). ${ }^{(c)}$ Odin results; Wirström et al. (2010). ${ }^{(d)}$ From simultaneous Accelerated Lambda Iteration (ALI) modelling of $\mathrm{H}_{2} \mathrm{O}, \mathrm{H}_{2}^{18} \mathrm{O}$, and $\mathrm{H}_{2}^{17} \mathrm{O}$ absorption lines. ${ }^{(e)}$ From ${ }^{15} \mathrm{NH}_{3}$ and assuming $N\left(\mathrm{H}_{2}\right)=1.5 \times 10^{24} \mathrm{~cm}^{-2}$ (cf. Nummelin et al. 2000, Sect. 5.1.1). ${ }^{(f)}$ Lower/upper limits from $\mathrm{H}_{2}^{18} \mathrm{O}$ absorption/invisible $\mathrm{H}_{2}^{17} \mathrm{O}$ absorption. ${ }^{(g)}$ Herschel absorption lines, Persson et al. ( 2010, 2012), average value and range of multi-velocity-component fits. ${ }^{(h)}$ Arecibo data; Plume et al. (2004). ${ }^{(i)}$ SWAS data; Plume et al. (2004), lower/upper limits from $\mathrm{H}_{2} \mathrm{O}$ absorption/invisible $\mathrm{H}_{2}^{18} \mathrm{O}$ absorption. ${ }^{(j)}$ Herschel absorption lines; Persson et al. (2012), average value and range of multi-velocity-component fits. ${ }^{(k)}$ Odin results; Wirström et al. (2006). ${ }^{(l)}$ Odin spectral line survey; Persson et al. (2007). ${ }^{(m)}$ Odin result; Larsson et al. (2003). ${ }^{(n)}$ ISO result; Goicoeachea (2008); Lerate et al. (2006). ${ }^{(o)}$ SWAS result from Monte Carlo modelling; Ashby et al. (2000). ${ }^{(p)}$ Odin result; Liseau et al. (2003). ${ }^{\left({ }^{q}\right)}$ Odin result from ALI modelling; Hjalmarson et al. (2003).

modelling of the outflow regions involves shock heating releasing molecules already deposited in grain surfaces as well as high temperature shock chemistry (for a short discussion and references, see Persson et al. 2007).

As pointed out in Sect. 3.2, the $557 \mathrm{GHz} \mathrm{H}_{2} \mathrm{O}$ emission line has extremely high optical depth complicating the derivation of physical parameters of the emitting regions. However, most of the spectral line profiles presented for the +50 and $+20 \mathrm{~km} \mathrm{~s}^{-1}$ clouds and the CND in Fig. 9 show broad wings. Whereas the blue wings are often confused by either self-absorption and/or spiral arm absorption, the red wings are generally uncontaminated and we here attempt to determine the water abundance in the regions giving rise to these red wings. We make our analysis using the on-line version of $\mathrm{RADEX}^{4}$ (van der Tak et al. 2007). We choose a line width of $60 \mathrm{~km} \mathrm{~s}^{-1}$ in the velocity intervals of 100 to $160 \mathrm{~km} \mathrm{~s}^{-1}$ and 50 to $110 \mathrm{~km} \mathrm{~s}^{-1}$ for the +50 and $+20 \mathrm{~km} \mathrm{~s}^{-1}$ clouds, respectively. These velocity intervals are chosen to ensure avoidance of the high optical depth cores of the respective $\mathrm{H}_{2} \mathrm{O}$ profiles. First we apply RADEX to the three $\mathrm{CO}$ lines $(J=7-6,5-4$, and $4-3)$ and are guided initially by the alternative model for the +50 and $+20 \mathrm{~km} \mathrm{~s}^{-1}$ clouds of Walmsley et al. (1986), which gives a kinetic temperature, $T_{\mathrm{k}}$ of $80 \mathrm{~K}$, and two density components: $80 \%$ with a density of

\footnotetext{
4 http://www.sron.rug.nl/ vdtak/radex/radex.php
}

$10^{4} \mathrm{~cm}^{-3}$, and $20 \%$ with a density of $10^{5} \mathrm{~cm}^{-3}$. The best fitting to our red $\mathrm{CO}$ wings (which would be the signature of the second component) then gives $T_{\mathrm{k}}=80 \mathrm{~K}$ and $n_{\mathrm{H}_{2}}=3 \times 10^{5} \mathrm{~cm}^{-3}$ for the $+50 \mathrm{~km} \mathrm{~s}^{-1}$ cloud and $T_{\mathrm{k}}=80 \mathrm{~K}$ and $n_{\mathrm{H}_{2}}=5 \times 10^{4} \mathrm{~cm}^{-3}$ for the $+20 \mathrm{~km} \mathrm{~s}^{-1}$ cloud. This results in $N\left(\mathrm{H}_{2}\right)=1.5 \times 10^{20}$ and $10^{21} \mathrm{~cm}^{-2}$, respectively, for the red wing components of the two clouds, assuming $X(C O)=10^{-4}$ (Dickman 1978; Lacy et al. 1994). With the same line width intervals, $T_{\mathrm{k}}$ and $n_{\mathrm{H}_{2}}$, applying RADEX to our $\mathrm{H}_{2} \mathrm{O}$ red line wings, we get $N\left(\mathrm{H}_{2} \mathrm{O}\right)=$ $1.15 \times 10^{14}$ and $10^{15} \mathrm{~cm}^{-2}$ for the +50 and $+20 \mathrm{~km} \mathrm{~s}^{-1}$ clouds, respectively. Finally, we obtain values for the water abundances in the red wing components for the +50 and $+20 \mathrm{~km} \mathrm{~s}^{-1}$ clouds of $X\left(\mathrm{H}_{2} \mathrm{O}\right)=10^{-6}$ and $8 \times 10^{-7}$, respectively.

For the CND, we performed a RADEX analysis using the velocity interval of 80 to $150 \mathrm{~km} \mathrm{~s}^{-1}$ in order to enable a comparison with the multi-CO-line SOFIA/GREAT observations of Requena-Torres et al. (2012). The best fitting gives $T_{\mathrm{k}}=160 \mathrm{~K}$, $n_{\mathrm{H}_{2}}=3 \times 10^{4} \mathrm{~cm}^{-3}$ and $N\left(\mathrm{H}_{2}\right)=7 \times 10^{20} \mathrm{~cm}^{-2}$, values quite compatible with the "low excitation" gas phase CND-N results of Requena-Torres et al. (2012). Again using the same line width, $T_{\mathrm{k}}$ and $n\left(\mathrm{H}_{2}\right)$ and applying RADEX to our $\mathrm{H}_{2} \mathrm{O}$ red line wing we get $N\left(\mathrm{H}_{2} \mathrm{O}\right)=4 \times 10^{15} \mathrm{~cm}^{-2}$ and thus a water abundance in the CND red wing component of $X\left(\mathrm{H}_{2} \mathrm{O}\right)=5.7 \times 10^{-6}$. The red wing results are also presented in Table 11 . These beamaveraged, but already high, $\mathrm{H}_{2} \mathrm{O}$ red line wing abundances may 
in fact be considered lower limits, since very likely the CO gas is abundant and is sufficiently excited in more extended regions than is the water vapour gas within the large $(2 \operatorname{arcmin})$ Odin antenna beam - leading to an overestimation of the comparison $\mathrm{H}_{2}$ column.

These high-velocity line wing water abundances are orders of magnitude higher than those estimated for the molecular cloud cores of the CND, as well as for the +50 and $+20 \mathrm{~km} \mathrm{~s}^{-1}$ clouds presented in Table 11 , and are very similar to the $\mathrm{H}_{2} \mathrm{O}$ abundance in the Orion KL low velocity outflow (Table 11) and also in the DR21 outflow (determined from Herschel HIFI data; van der Tak et al. 2010) and suggested to be the result of desorption of water ice deposited on grain surfaces. This scenario also has been proposed to explain the high abundance of gas phase $\mathrm{SiO}$ observed in the $\mathrm{Sgr}$ A region (Amo-Baladrón et al. 2011). The even higher water abundance observed in the Orion KL high velocity outflow (Odin results in Table 11, consistent with multi-transition Herschel HIFI data; Melnick et al. 2010), most likely is the result of the combined action of shock desorption of grain mantles and high temperature gas-phase shock chemistry.

ii) Hydroxyl radical. According to Eq. (3) the $\mathrm{OH}$ column densities, and hence the $\mathrm{OH}$ abundances, are linearly dependent on the estimated excitation temperatures $T_{\text {ex }}(\mathrm{OH})$. Since we feel that the estimated excitation temperatures should be true to within a factor of 2 , this also should be true for the estimated $\mathrm{OH}$ column densities and abundances.

According to Table 11, the $\mathrm{OH}$ abundance appears to be enhanced by an order of magnitude in the CND cloud core and is very likely also enhanced in the +50 and $+20 \mathrm{~km} \mathrm{~s}^{-1}$ cloud cores, all compared to the abundances estimated for the spiral arm clouds observed against Sgr A and W49N and also compared to the $\mathrm{OH}$ abundance in the Sgr B2 cloud envelope. In case of Orion the multi-line $\mathrm{OH}$ absorptions and emissions observed by ISO have been assigned to the molecular outflow (cf. Lerate et al. 2006; Goicoechea 2008). The enhanced $\mathrm{OH}$ abundances in the Sgr A molecular clouds could be the results of shock and photo dissociation of $\mathrm{H}_{2} \mathrm{O}$, decreasing the high gas-phase $\mathrm{H}_{2} \mathrm{O}$ abundances produced by shock and photo desorption from icy grain mantles.

We note in passing that the estimated $\mathrm{OH}$ abundances listed in Table 11 (although admittedly uncertain), seem to indicate an $\mathrm{OH}$ abundance increase with decreasing distance from the Galactic centre. The reason of which still is uncertain, but could partly be an oxygen elemental abundance increase caused by stellar nucleosynthesis.

iii) Ammonia. The ortho-ammonia abundances determined from the absorption lines emanating in the spiral arm foreground clouds are quite similar $\left(3-5 \times 10^{-9}\right)$ and are also similar to the value estimated from the relatively strong $\mathrm{NH}_{3}$ emission line observed by Odin in the Orion KL bar $\left(5 \times 10^{-9}\right.$; Larsson et al. 2003). These abundances are definitely higher than the one estimated from the narrow velocity $\mathrm{NH}_{3}$ emission line observed by Odin in the low mass cloud core rho Oph A $\left(4 \times 10^{-10}\right.$; Liseau et al. 2003). All these $\mathrm{NH}_{3}$ abundances appear to be accommodated within the gas-phase chemistry models of Pineau des Forêts et al. (1990), where the lowest observed value may be explained as a result of dynamical mixing between a carbon rich cloud envelope and the cloud core. The $\mathrm{NH}_{3}$ abundances in the PDRs may also contain contributions from grain surface detachment and grain surface chemistry (cf. our comments on $\mathrm{H}_{2} \mathrm{O}$ ). This is definitely the case for the Orion KL compact ridge and hot core sources, where the ammonia abundances have been estimated to be $2 \times 10^{-7}$ and $2 \times 10^{-6}$, respectively (using the Odin spectral line survey of Orion KL); for analysis and chemistry discussion, see Persson et al. (2007).

\section{Conclusions}

We have observed the three major Giant molecular clouds in the Sgr A complex at the GC, the expanding molecular ring and the line-of-sight spiral arm features with the VLA and SEST telescopes, and the Odin satellite. Four molecules $\left(\mathrm{OH}, \mathrm{H}_{2} \mathrm{O}, \mathrm{NH}_{3}\right.$, $\mathrm{CO})$, and one atomic specimen $\left(\mathrm{C}_{\mathrm{I}}\right)$, were studied at certain energy transitions in these objects. $\mathrm{OH}, \mathrm{H}_{2}^{18} \mathrm{O}$, and $\mathrm{NH}_{3}$ were detected in absorption, $\mathrm{CO}, \mathrm{C}^{18} \mathrm{O}$, and $\mathrm{C}_{\mathrm{I}}$, in emission. $\mathrm{H}_{2} \mathrm{O}$ was detected in emission as well as in absorption. Column densities and abundances have been determined for these species in the different objects.

- The $\mathrm{OH}$ abundance appears to be enhanced by an order of magnitude in the CND cloud core and is very likely also enhanced in the +50 and $+20 \mathrm{~km} \mathrm{~s}^{-1}$ cloud cores, all compared to the abundances estimated for the spiral arm clouds observed against Sgr A and W49N and also compared to the $\mathrm{OH}$ abundance in the Sgr B2 cloud envelope.

- As compared to the quiescent cloud values of a few $\times 10^{-9}$, or lower, the $\mathrm{H}_{2} \mathrm{O}$ abundance is markedly enhanced in the front sides of the Sgr A molecular cloud cores, $(2-7) \times 10^{-8}$, as observed in absorption and being highest in the CND. A similar abundance enhancement is seen in $\mathrm{OH}$. An unusually high $\mathrm{OH} / \mathrm{H}_{2} \mathrm{O}$ ratio is also found in the clouds at the GC. The likely explanation is PDR chemistry including grain surface reactions, and perhaps also influences of shocks and turbulence in clouds.

- In the redward high-velocity line wings of the +50 and $+20 \mathrm{~km} \mathrm{~s}^{-1}$ clouds and CND, the $\mathrm{H}_{2} \mathrm{O}$ abundances are estimated to be $(1-6) \times 10^{-6}$, or higher, i.e., similar to the water abundances in the outflows of the Orion KL and DR21 molecular clouds, suggested to be caused by the combined action of shock desorption from icy grain mantles and high temperature gas phase shock chemistry.

- Three large clumps were identified in the $+50 \mathrm{~km} \mathrm{~s}^{-1}$ cloud in $\mathrm{C}^{18} \mathrm{O} J=2-1$ emission, the surfaces of which appear to be associated with $1720 \mathrm{MHz} \mathrm{OH}$ masers.

- The position-velocity cut of the $\mathrm{C}^{18} \mathrm{O}$ emission through the compact $\mathrm{H}$ in region $\mathrm{D}$, indicates the existence of a large-scale two-component structure in this source.

- An area of depleted $\mathrm{C}^{18} \mathrm{O}$ emission was found in the molecular belt between the +50 and $+20 \mathrm{~km} \mathrm{~s}^{-1}$ clouds, at a location near the SNR (G359.92-0.09).

- A wide velocity feature was observed in $\mathrm{OH}$ absorption centred at $-32 \mathrm{~km} \mathrm{~s}^{-1}$, and about $107 \mathrm{~km} \mathrm{~s}^{-1}$ wide, in the same area as a previously observed blue-shifted high-velocity cloud in $\mathrm{C}^{18} \mathrm{O}$ emission.

Acknowledgements. The authors acknowledge the open policy for the use of NRAO's VLA. The National Radio Astronomy Observatory (NRAO) is operated by Associated Universities Inc., under cooperative agreement with the National Science Foundation. We also acknowledge with gratitude the support from Miller Goss with concatenation of the observations with the VLA in its BnA and DnC configurations. Furthermore, the extensive support from the Swedish National Space Board (Rymdstyrelsen) is gratefully acknowledged by Aage Sandqvist, Åke Hjalmarson, and Michael Olberg. Kambiz Fathi acknowledges support from the Swedish Research Council (Vetenskapsrådet) and the Swedish Royal Academy of Sciences' Crafoord Foundation. Special thanks also to Bengt Larsson for discussions and the development of the Odin Reduction Package (ORP). Furthermore, the authors would like to thank the anonymous referee and the editor for the detailed and very constructive comments which greatly improved the presentation of our results and conclusions. 


\section{Appendix A: SEST observations of $C^{18} O J=2-1$ towards the Sgr A complex}

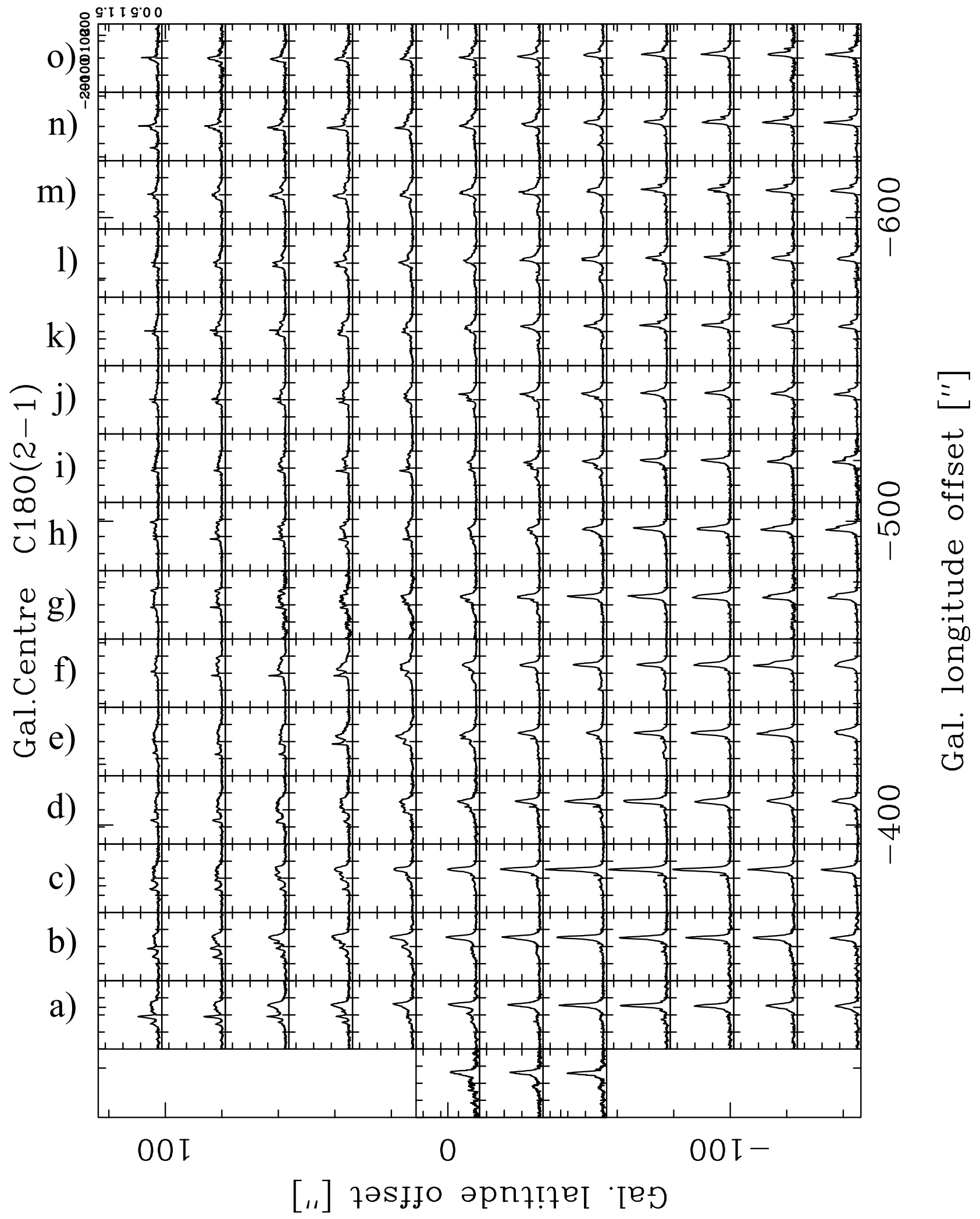

Fig. A.1. SEST observations of $\mathrm{C}^{18} \mathrm{O} J=2-1$ towards the Sgr A complex. The offsets (in arcseconds) are in the Galactic coordinate system and are relative to the position $(l, b)=\left(+5^{\prime},-3^{\prime}\right)$. The letters to the left of the diagram indicate the positions of the position-velocity cuts in Figs. B.1a-o. The velocity range is -200 to $+200 \mathrm{~km} \mathrm{~s}^{-1}$ and the antenna temperature range is -0.1 to $+1.7 \mathrm{~K}$. 
Appendix B: Position-velocity diagrams of $C^{18} \mathrm{O}=2-1$ observations.
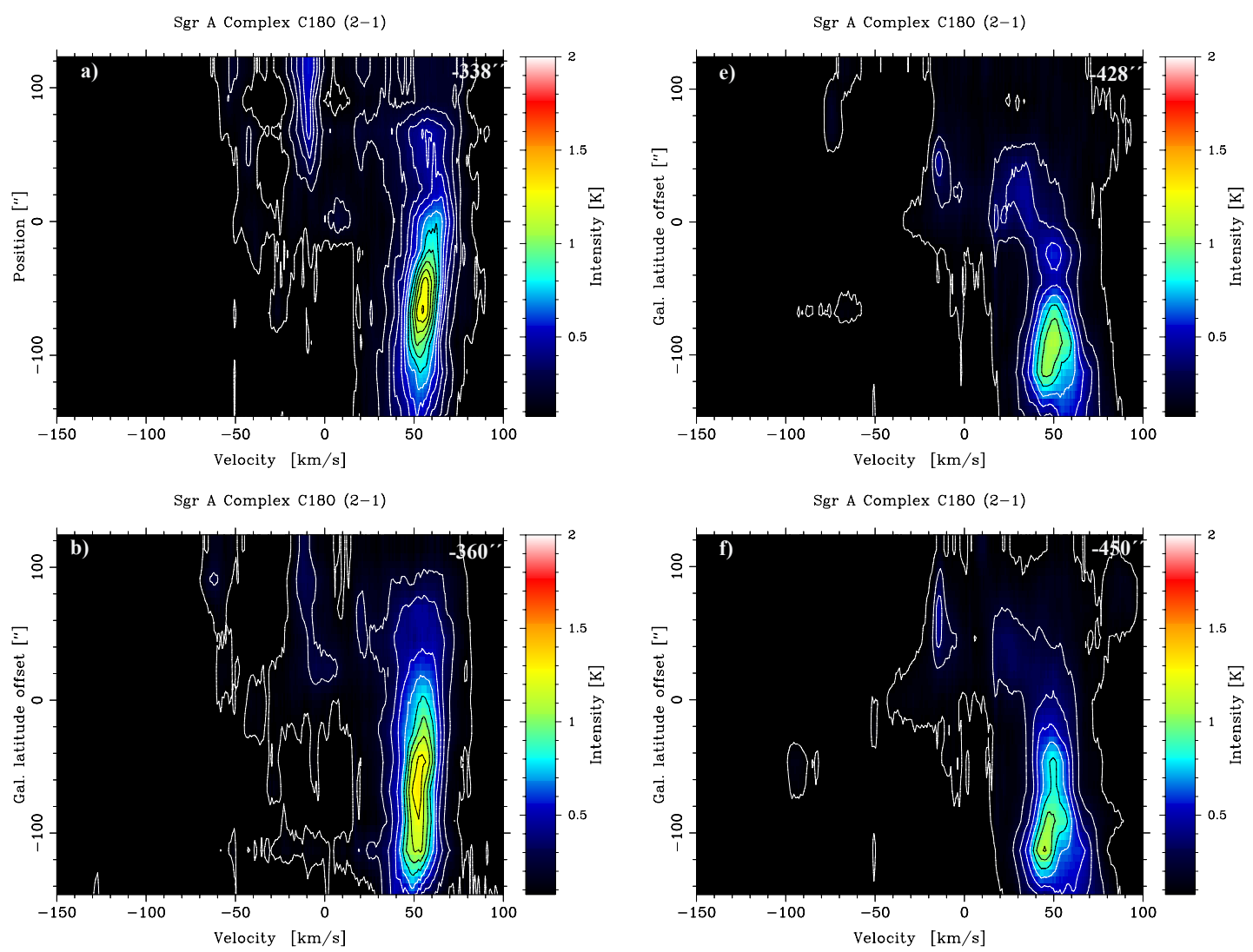

Sgr A Complex C180 (2-1)

Sgr A Complex C180 (2-1)
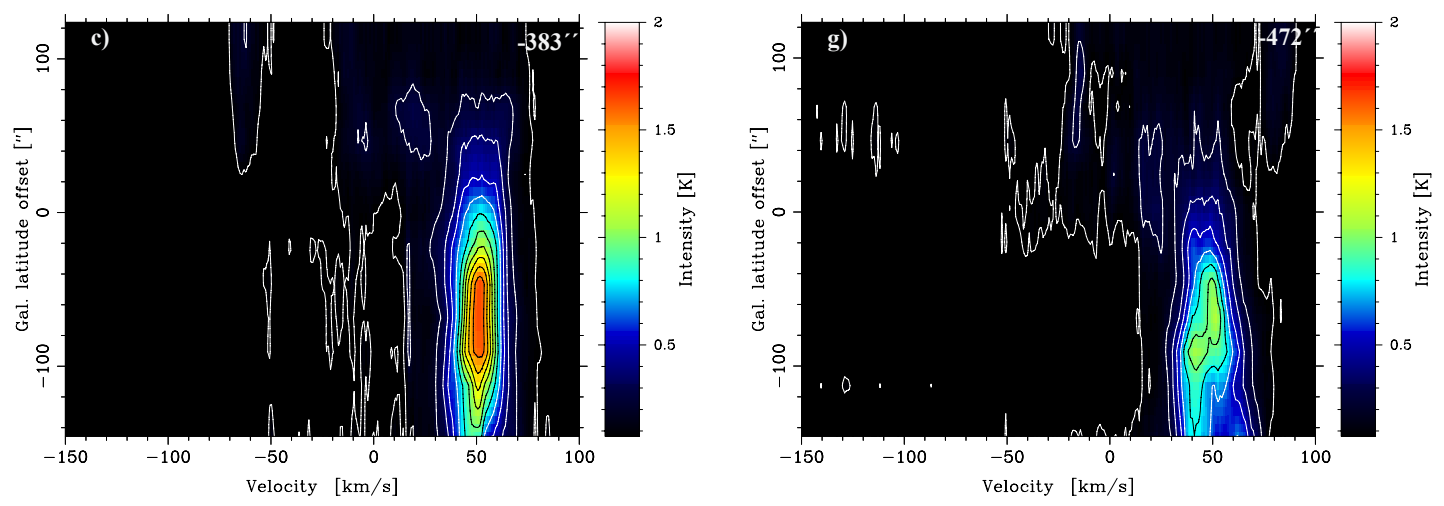

Sgr A Complex C180 (2-1)

Sgr A Complex C180 (2-1)
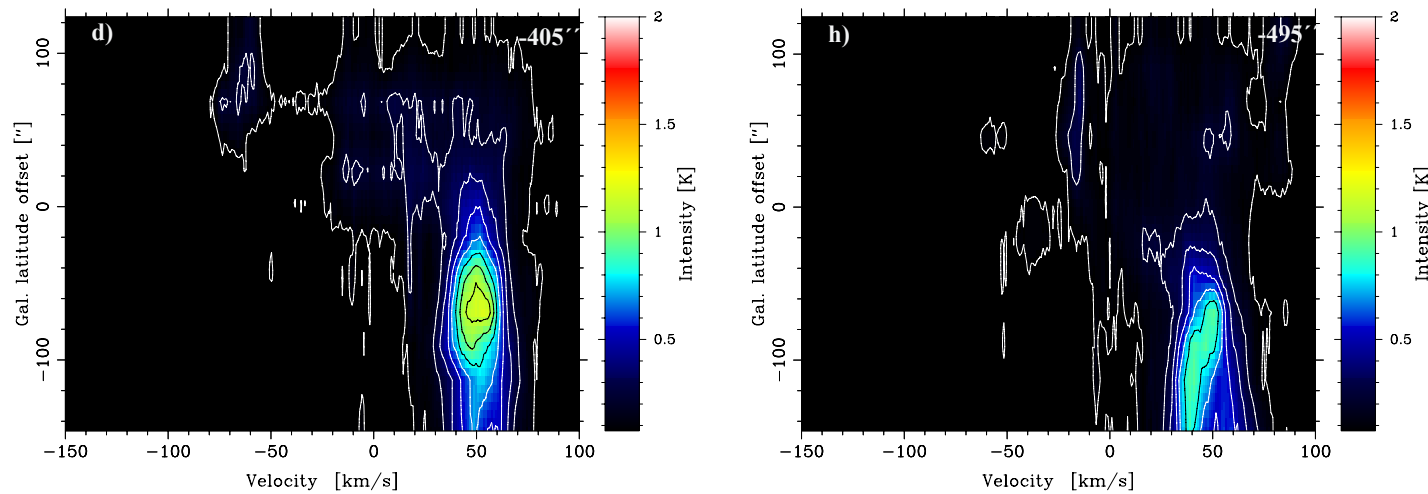

Fig. B.1. a)-h). $\mathrm{C}^{18} \mathrm{O} J=2-1$ position-velocity diagrams at constant Galactic longitude offset from $(l, b)=\left(+5^{\prime},-3^{\prime}\right)$, which is noted in the right hand upper corner of each figure. The letter in the left hand upper corner of the figures corresponds to the respective row of profiles in Fig. A.1. Note that the figures in the panel should be read from top to bottom in each column. The direction of the position-velocity cuts are parallel to the lines $(\mathrm{C}-\mathrm{D}),(\mathrm{E}-\mathrm{F})$, and $(\mathrm{G}-\mathrm{H})$ in Fig. 11. The offset latitude of Sgr A* is 14". The lowest contour level corresponds to about $2 \sigma$. 

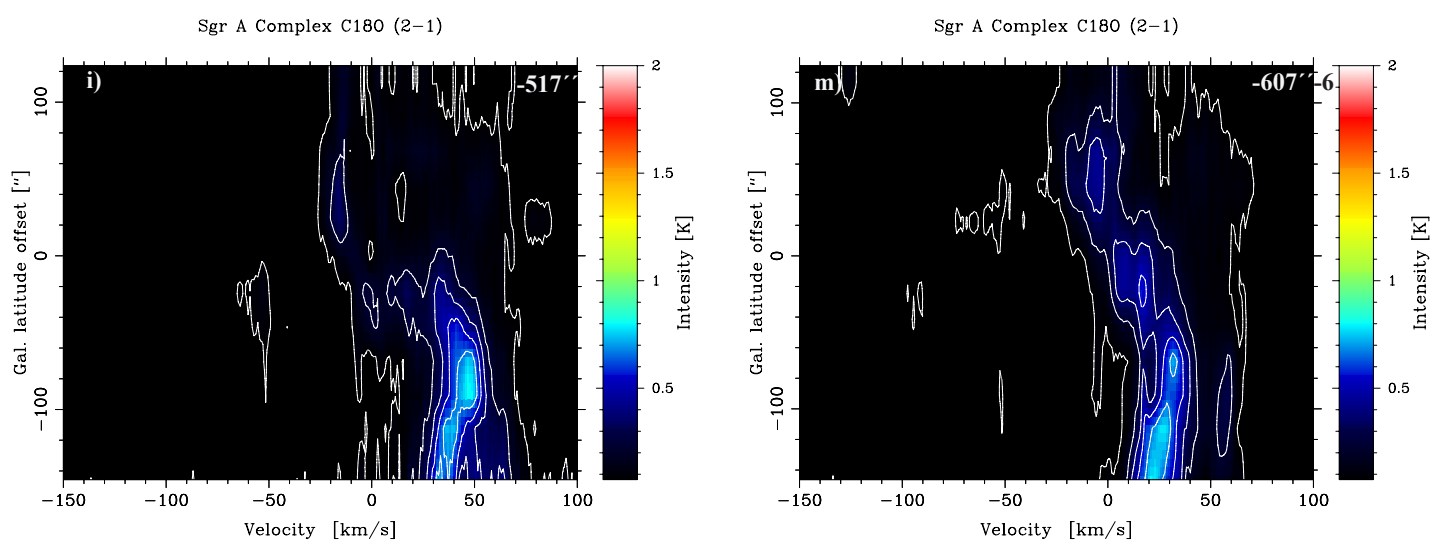

Sgr A Complex C180 (2-1)

Sgr A Complex C180 (2-1)
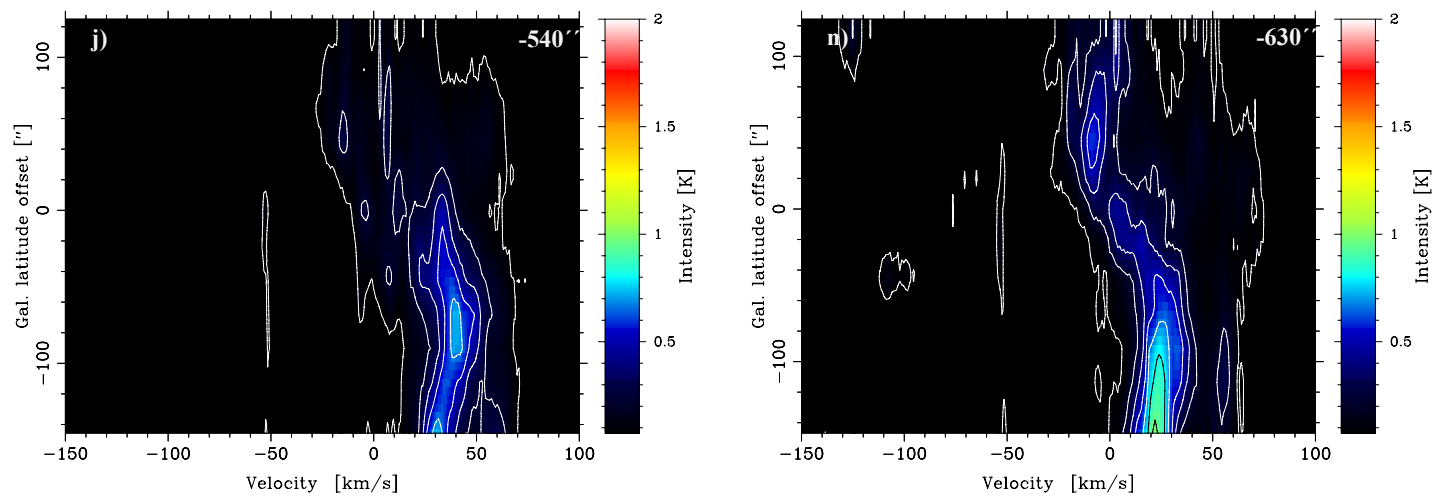

Sgr A Complex C180 (2-1)

Sgr A Complex C180 (2-1)
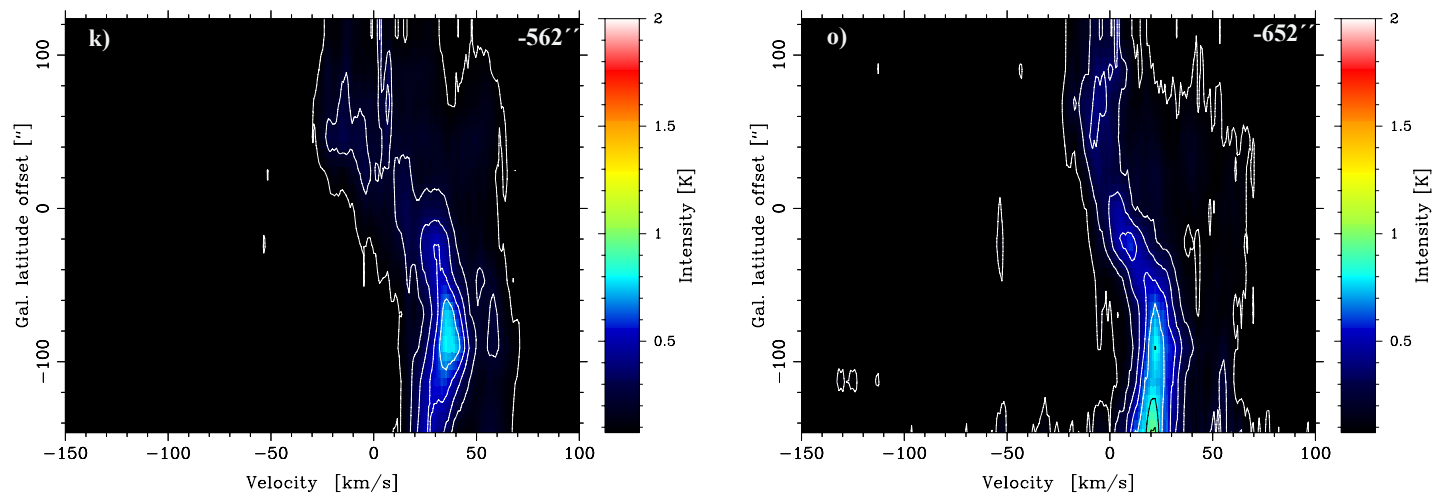

Sgr A Complex C180 (2-1)

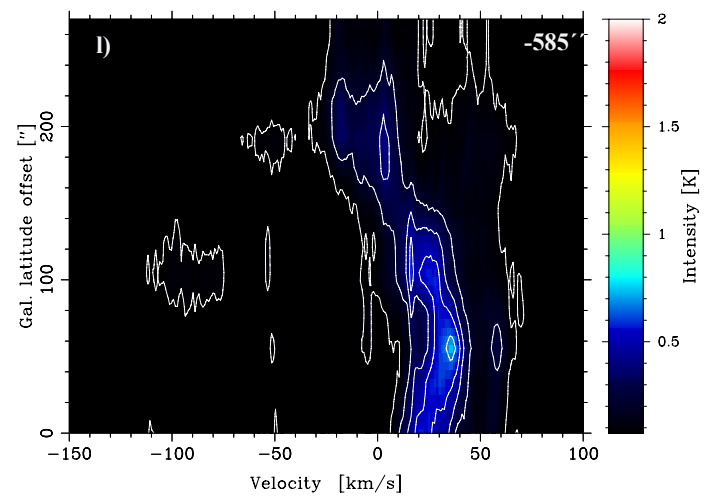

Fig. B.2. i) $-\mathbf{o})$. $\mathrm{C}^{18} \mathrm{O} J=2-1$ position-velocity diagrams at constant Galactic longitude offset from $(l, b)=\left(+5^{\prime},-3^{\prime}\right)$, which is noted in the right hand upper corner of each figure. The letter in the left hand upper corner of the figures corresponds to the respective row of profiles in Fig. A.1. Note that the figures in the panel should be read from top to bottom in each column. The direction of the position-velocity cuts are parallel to the lines $(\mathrm{C}-\mathrm{D}),(\mathrm{E}-\mathrm{F})$, and $(\mathrm{G}-\mathrm{H})$ in Fig. 11. The offset latitude of Sgr A* is 14". The lowest contour level corresponds to about $2 \sigma$. 


\section{References}

Amo-Baladrón, M. A., Martin-Pintado, J., \& Martin, S. 2011, A\&A, 526, A54 Ashby, M. L. N., Bergin, E. A., Plume, R., et al. 2000, ApJ, 539, L119 Bally, J. 1989, ESOC 33, 1

Bally, J., Stark, A. A., Wilson, R. W., \& Henkel, C. 1988, ApJ, 324, 223 Becklin, E. E., Gatley, I., \& Werner, M. W. 1982, ApJ, 258, 135 Bergin, E. A., Melnick, G. J., Stauffer, J. R., et al. 2000, ApJ, 539, L129

Burton, W. B. 1988, in Galactic and Extragalactic Astronomy, eds. G. L. Verschur, \& K. I. Kellermann, 295

Coil, A. L., \& Ho, P. T. P. 2000, ApJ, 533, 245

Dickman, R. L. 1978, ApJS, 37, 407

Dowell, C. D., Lis, D. C., Serabyn, E., et al. 1999, in The Central Parsecs of the Galaxy, eds. H. Falcke et al., ASP Conf. Ser., 186, 453

Ekers, R. D., van Gorkom, J. H., Schwarz, U. J., \& Goss, W. M. 1983, A\&A, 122,143

Elitzur, M., \& de Jong, T. 1978, A\&A, 67, 323

Ferrière, K. 2012, A\&A, 540, A50

Frisk, U., Hagström, M., Ala-Laurinaho, J., et al. 2003, A\&A, 402, L27

Genzel, R., Stacey, G. J., Harris, A. I., et al. 1990, ApJ, 356, 160

Ghez, A. M., Salim, S., Weinberg, N. N., et al. 2008, ApJ, 689, 1044

Goicoechea, J. R. 2008, EAS Publ. Ser., 31, 73

Goldsmith, P. F. 1999, Millimeter-Wave Astronomy: molecular Chemistry \& Physics in Space, eds. W. F. Wall et al., ASSL, 241, 57

Goss, W. M. 1968, ApJS, 15, 131

Goss, W. M., Schwarz, U. J., van Gorkom, J. H., et al. 1985, MNRAS, 215, 69

Güsten, R., \& Downes, D. 1981, A\&A, 99, 27

Herrnstein, R. M., \& Ho, P. T. P. 2005, ApJ, 620, 287

Hjalmarson, A., Frisk, U., Olberg, M., et al. 2003, A\&A, 402, L39

Hollenbach, D., Kaufman, M. J., Bergin, E. A., \& Melnick, G. J. 2009, ApJ, 690,1497

Jackson, J. M., Geis, N., Genzel, R., et al. 1993, ApJ, 402, 173

Karlsson, R., Sjouwerman, L. O., Sandqvist, A., \& Whiteoak, J. B. 2003, A\&A, 403, 1011

Keene, J., Blake, G. A., \& Phillips, T. G. 1983, ApJ, 271, L27

Lacy, J. H., Knacke, R., Geballe, T. R., \& Tokunaga, T. 1994, ApJ, 428, L69

Larsson, B., Liseau, R., Bergman, P., et al. 2003, A\&A, 402, L69

Lerate, M. R., Barlow, M. J., Swinyard, B. M., et al. 2006, MNRAS, 370, 597

Lindqvist, M., Sandqvist, Aa., Winnberg, A., Johansson, L. E. B., \& Nyman, L.-A. 1995, A\&AS, 113, 257

Lis, D. C., \& Carlstrom, J. E. 1994, ApJ, 424, 189

Liseau, R., Larsson, B., Brandeker, A., et al. 2003, A\&A, 402, L73

Lockett, P., Gauthier, E., \& Elitzur, M. 1999, ApJ, 511, 235

Martin, C. L., Walsh, W. M., Xiao, K., et al. 2004, ApJS, 150, 239

Martín, S., Martín-Pintado, J., Montero-Castaño, M., Ho, P. T. P., \& Blundel, R. 2012, A\&A, 539, A29

Melnick, G. J., Tolls, V., Neufeld, D. A., et al. 2010, A\&A, 512, L27

Mezger, P. G., Duschl, W. J., \& Zylka, R. 1996, A\&ARv, 7, 289

Mills, E., Morris, M. R., Lang, C. C., et al. 2011, ApJ, 735, 84

Miyazaki, A., \& Tsuboi, M. 1999, in Star Formation 99, Proc., 106

Morris, M., \& Serabyn, E. 1996, ARA\&A, 34, 645

Neufeld, D. A., Snell, R. L., Ashby, L. N., et al. 2000, ApJ, 539, L107

Neufeld, D. A., Kaufman, M. J., Goldsmith, P. F., Holenbach, D. J., \& Plume, R. 2002, ApJ, 580, 278

Neufeld, D. A., Bergin, E. A., Melnick, G. J., \& Goldsmith, P. F. 2003, ApJ, 590, 882
Nummelin, A., Bergman, P., Hjalmarson, Å., et al. 2000, ApJS, 128, 213

Oka, T., Geballe, T. R., Goto, M., Usuda, T., \& McCall J. 2005, ApJ, 632, 882

Olberg, M., Frisk, U., Lecacheux, A., et al. 2003, A\&A, 402, 35

Olofsson, A. O. H., Olofsson, G., Hjalmarson, A., et al. 2003, A\&A, 402, L47

Persson, C. M., Olofsson, A. O. H., Koning, N., et al. 2007, A\&A, 476, 807

Persson, C. M., Black, J. H., Cernicharo, J., et al. 2010, A\&A, 521, L45

Persson, C. M., de Luca, M., Mookerjea, B., et al. 2012, A\&A, 543, A145

Phillips, T. R., \& Green, S. 1995, Ap\&SS, 224, 537

Philllips, T. G., \& Huggins, P. J. 1981, ApJ, 251, 533

Pineau des Forêts, G., Roeff, E., \& Flower, D. R. 1990, MNRAS, 244, 668

Plume, R., Kaufman, M. J., Neufeld, D. A., et al. 2004, ApJ, 605, 247

Poglitsch, A., Stacey, G. J., Geis, N., et al. 1991, ApJ, 374, L33

Polehampton, E. T., Baluteau, J. P., Swinyard, B. M., et al. 2007, MNRAS, 377, 1122

Requena-Torres, M. A., Güsten, R., Weiß, A., et al. 2012, A\&A, 542, L21

Rodríguez-Fernández, N. J., Martín-Pintado, J., Fuente, A., et al. 2001, A\&A, 365,174

Sandqvist, Aa. 1974, A\&A, 33, 413

Sandqvist, Aa. 1989, A\&A, 223, 293

Sandqvist, Aa., Bergman, P., Black, J., et al. 2003, A\&A, 402, L63

Sandqvist, Aa., Bergman, P., Bernath, P., et al. 2006, in From the Center of the Milky Way to Nearby Low-Luminosity Galactic Nuclei, eds. R. Schödel et al., J. Phys. Conf. Ser., 54, 72

Schödel, R., Ott, T., Genzel, R., et al. 2002, Nature, 419, 694

Serabyn, E., Lacy, J. H., \& Achtermann, J. M. 1992, ApJ, 395, 166

Sjouwerman, L. O., \& Pihlström, Y. M. 2008, ApJ, 681, 1287

Snell, R. L., Howe, J. E., Ashby, M. L. N., et al. 2000, ApJ, 539, L101

Spaans, M., Neufeld, D., Lepp, S., Melnick, G. J., \& Stauffer, J. 1998, ApJ, 503, 780

Tanaka, K., Oka, T., Matsumura, S., et al. 2011, ApJ, 743, L39

Tsuboi, M., Miyazaki, A., \& Okumura, S. K. 2009, PASJ, 61, 29

Tsuboi, M., Tadaki, K., Miyazaki, A., \& Handa, T. 2011, PASJ, 63, 763

van der Tak, F. F. S., Black, J. H., Schöier, F. L., Jansen, D. J., \& Dishoeck, E. F. 2007, A\&A, 468, 627

van der Tak, F. F. S., Marseille, M. G., Herpin, F., et al. 2010, A\&A, 581, L107

Vejby-Christensen, L., Andersen, L. H., Heber, O., et al. 1997, ApJ, 483, 531

Walmsley, C. M., Güsten, R., Angerhofer, P., \& Mundy, L. 1986, A\&A, 155, 129

Wilson, T. L., \& Rood, R. 1994, ARA\&A, 32, 191

Wilson, C. D., Mason, A., Gregersen, E., et al. 2003, A\&A, 402, L59

Winnewisser, M., Winnewisser, B. P., \& Winnewisser, G. 1985, in molecular Astrophysics, Series C, 157, eds. G. H. F. Diercksen, W. F. Huebner, \& P. W. Langhoff, 375

Wirström, E. S., Bergman, P., Olofsson, A. O. H., et al. 2006, A\&A, 453, 979

Wirström, E. S., Bergman, P., Black, J., et al. 2010, A\&A, 522, A19

Yusef-Zadeh, F., \& Morris, M. 1987, ApJ, 320, 545

Yusef-Zadeh, F., Lasenby, A., \& Marshall, J. 1993, ApJ, 410, L27

Yusef-Zadeh, F., Roberts, D. A., Goss, W., M., Frail, D. A., \& Green, A. J. 1996, ApJ, 466, L25

Yusef-Zadeh, F., Stolovy, S. R., Burton, M., Wardle, M., \& Ashley, M. C. B. 2001, ApJ, 560, 749

Yusef-Zadeh, F., Lacy, J. H., Wardle, M., et al. 2010, ApJ, 725, 1429

Zylka, R., Mezger, P. G., \& Wink, J. E. 1990, A\&A, 234, 133 